\title{
Extreme $\pi$-Loading as a Design Element for Accessing Imido Ligand Reactivity. A CCC-NHC Pincer Tantalum Bis(imido) Complex: Synthesis, Characterization, and Catalytic Oxidative Amination of Alkenes
}

Theodore R. Helgert,${ }^{a, b \dagger \xi}$ Xiaofei Zhang, ${ }^{a \S}$ Hannah K. Box, ${ }^{a \S}$ Jason A. Denny, ${ }^{a}$ Henry U. Valle, ${ }^{a}$ Allen G.

Oliver, ${ }^{c}$ Gopalakrishna Akurathi, ${ }^{a}$ Charles Edwin Webster,${ }^{a *}$ T. Keith Hollis, ${ }^{a, b *}$

${ }^{a}$ Department of Chemistry and Center for Computational Sciences, Mississippi State University, Mississippi State, Mississippi 39762, United States

bDepartment of Chemistry and Biochemistry, The University of Mississippi, Oxford, MS 38655, United States

${ }^{c}$ Department of Chemistry and Biochemistry, University of Notre Dame, Notre Dame, Indiana 46556, United States

\section{Content}

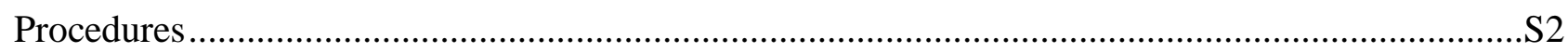

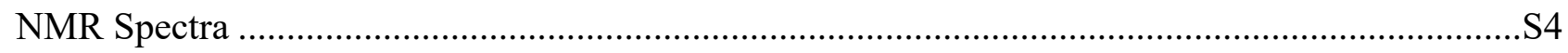

High Resolution Mass Spectrometry Data......................................................................................S39

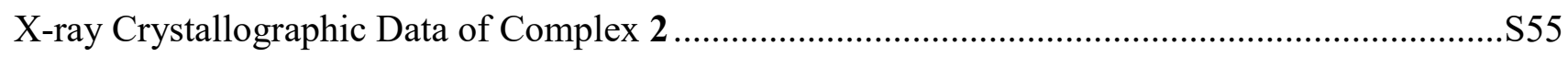

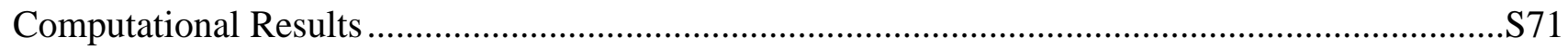




\section{Procedures}

Isolation of crystalline material.

1,3-bis(3-butylimidazol-1-yl-2-idene)-2-phenylene)(tert-butylimido)-(dimethylamido)iodotantalum(V) (0.350 g, $0.470 \mathrm{mmol})$, lithium tert-butylamide $(0.081 \mathrm{~g}, 1.03 \mathrm{mmol})$, and toluene $(15 \mathrm{~mL})$ were combined in a vial with a Teflon lined cap. The mixture was heated at $40{ }^{\circ} \mathrm{C}$ for $2 \mathrm{~h}$ and then cooled to room temperature for 6 days. Hexanes $(10 \mathrm{~mL})$ was then layered on to the crude reaction solution and allowed to sit at room temperature for $24 \mathrm{~h}$, where crystals grew. The mother liquor was decanted from the crystal. The crystals were washed with hexanes $(3 \times 3$ $\mathrm{mL})$ and the volatiles removed to yield off-white X-ray quality crystals, $2(0.092 \mathrm{~g}, 25 \%)$.

Time-dependence of the yields of oxidative amination, reduction, and hydroamination products (Table 2, entries 3, 4, and 5): (1,3-bis(3-butylimidazol-1-yl-2-idene)-2-phenylene)bis(t-butylimido)tantalum(V) lithium iodide bridged dimer $(2,0.0078 \mathrm{~g}, 0.0050 \mathrm{mmol},[2]=0.005 \mathrm{M}),(2,2$-diphenyl-4-pentenyl)amine $(0.0500 \mathrm{~g}, 0.21 \mathrm{mmol}$, [amine] $=0.21 \mathrm{M})$ and $\mathrm{C}_{6} \mathrm{D}_{6}(1.0 \mathrm{~mL})$ were combined in an NMR tube. The reaction was heated at $100{ }^{\circ} \mathrm{C}$ and monitored by ${ }^{1} \mathrm{H}$ NMR spectroscopy.

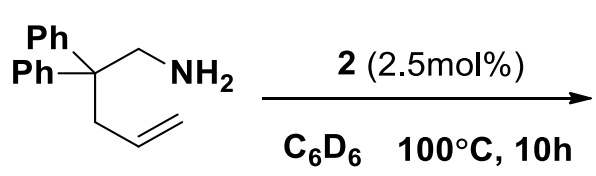

A

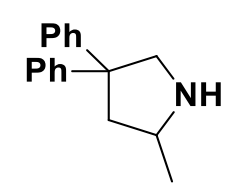

B

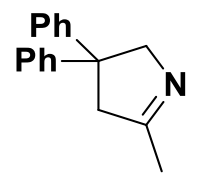

C

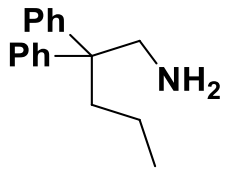

D

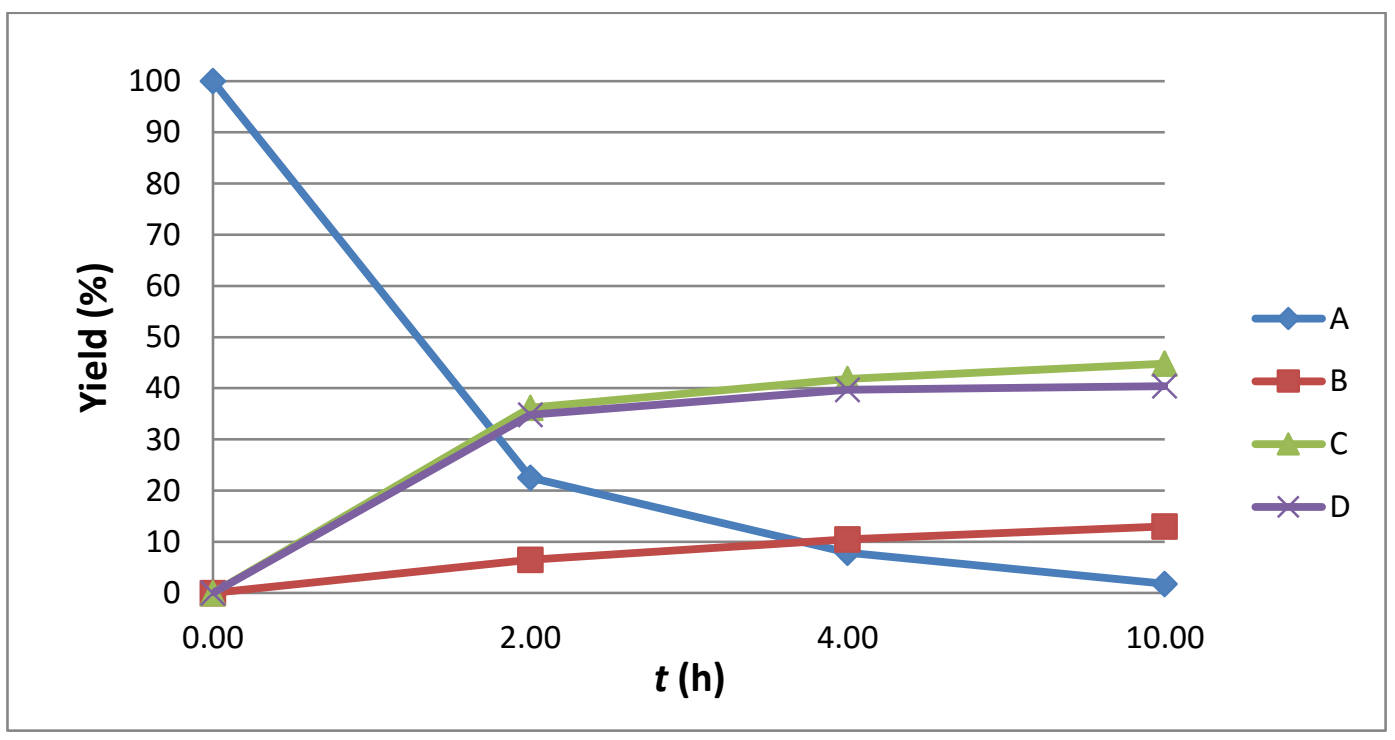

Scheme S1. Time-dependence of the yields of oxidative amination, reduction, and hydroamination products.

Concentration-dependence of the yields of oxidative amination, reduction, and hydroamination products (Table 2, entries 3, 6, 7, and 8): To four screw-capped NMR tubes complex 2 (tube $1 \& 2: 0.5 \mathrm{mg}, 0.0003 \mathrm{mmol}$, [2] = $0.0005 \mathrm{M}$; tube $3 \&$ 4: $5.0 \mathrm{mg}, 0.003 \mathrm{mmol}$, [2] = 0.005 M), (2,2-diphenyl-4-pentenyl)amine (tube $1 \& 3: 3.0 \mathrm{mg}$, 
$0.0126 \mathrm{mmol}$, [amine] $=0.021 \mathrm{M}$; tube $2 \& 4: 30.0 \mathrm{mg}, 0.126 \mathrm{mmol}$, [amine] $=0.21 \mathrm{M})$ and $\mathrm{C}_{6} \mathrm{D}_{6}(0.6 \mathrm{~mL})$ were added. The reactions were heated at $100{ }^{\circ} \mathrm{C}$ and monitored by ${ }^{1} \mathrm{H}$ NMR spectroscopy.

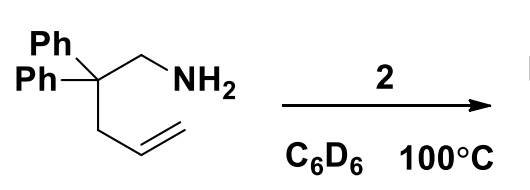

A

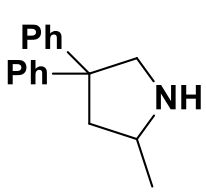

B

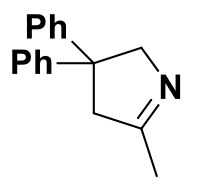

C

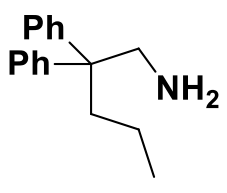

D

Table S1. Concentration-dependence of the yields of oxidative amination, reduction, and hydroamination products.

\begin{tabular}{|l|l|l|l|l|l|}
\hline$[2]$ & {$[$ amine $]$} & $\mathbf{\% B}$ & $\mathbf{\% C}$ & $\mathbf{\% D}$ & $\mathbf{B} / \mathbf{C}$ \\
\hline 0.001 & 0.021 & 4.3 & 54.9 & 40.8 & 0.078 \\
\hline 0.001 & 0.21 & NA & & & \\
\hline 0.01 & 0.021 & 2.3 & 57.7 & 40.0 & 0.040 \\
\hline 0.01 & 0.21 & 13.2 & 45.7 & 41.1 & 0.289 \\
\hline
\end{tabular}

Additive-dependence of the yields of oxidative amination, reduction, and hydroamination products (Table 2, entries 9, 10, and 11): To a screw-capped NMR tube complex 2 (0.5 mg, $0.0006 \mathrm{mmol}$, [2] = 0.001 M), (2,2diphenyl-4-pentenyl)amine $(3.0 \mathrm{mg}, 0.0126 \mathrm{mmol}$, [amine] $=0.021 \mathrm{M})$, additive $(0.0378 \mathrm{mmol}$, [additive] $=0.063$ $\mathrm{M})$ and $\mathrm{C}_{6} \mathrm{D}_{6}(0.6 \mathrm{~mL})$ were added. The reaction was heated at $100{ }^{\circ} \mathrm{C}$ and monitored by ${ }^{1} \mathrm{H}$ NMR spectroscopy.

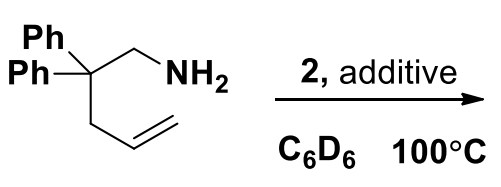

A

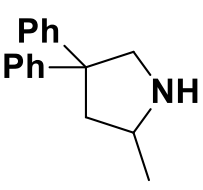

B

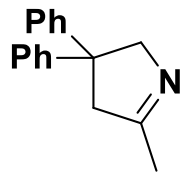

C

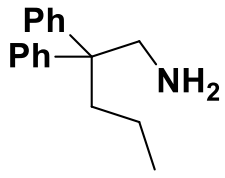

D

Table S2. Additive-dependence of the yields of oxidative amination, reduction, and hydroamination products

\begin{tabular}{|l|l|l|l|l|l|l|l|}
\hline$[2]$ & {$[$ amine $]$} & Additive & Cond. & HA & OA & Red & Conv. \\
\hline 0.001 & 0.021 & 9,10 -dihydroanthracene & $100^{\circ} \mathrm{C} 10 \mathrm{~h}$ & 8.6 & 51.3 & 40.1 & 97 \\
\hline & & TBE & $100^{\circ} \mathrm{C} 12 \mathrm{~h}$ & 3.1 & 52.4 & 44.5 & 78.3 \\
\hline & & 1 -hexene & $100^{\circ} \mathrm{C} 11 \mathrm{~h}$ & 0 & 10.7 & 0 & 10.7 \\
\hline
\end{tabular}


NMR spectra:

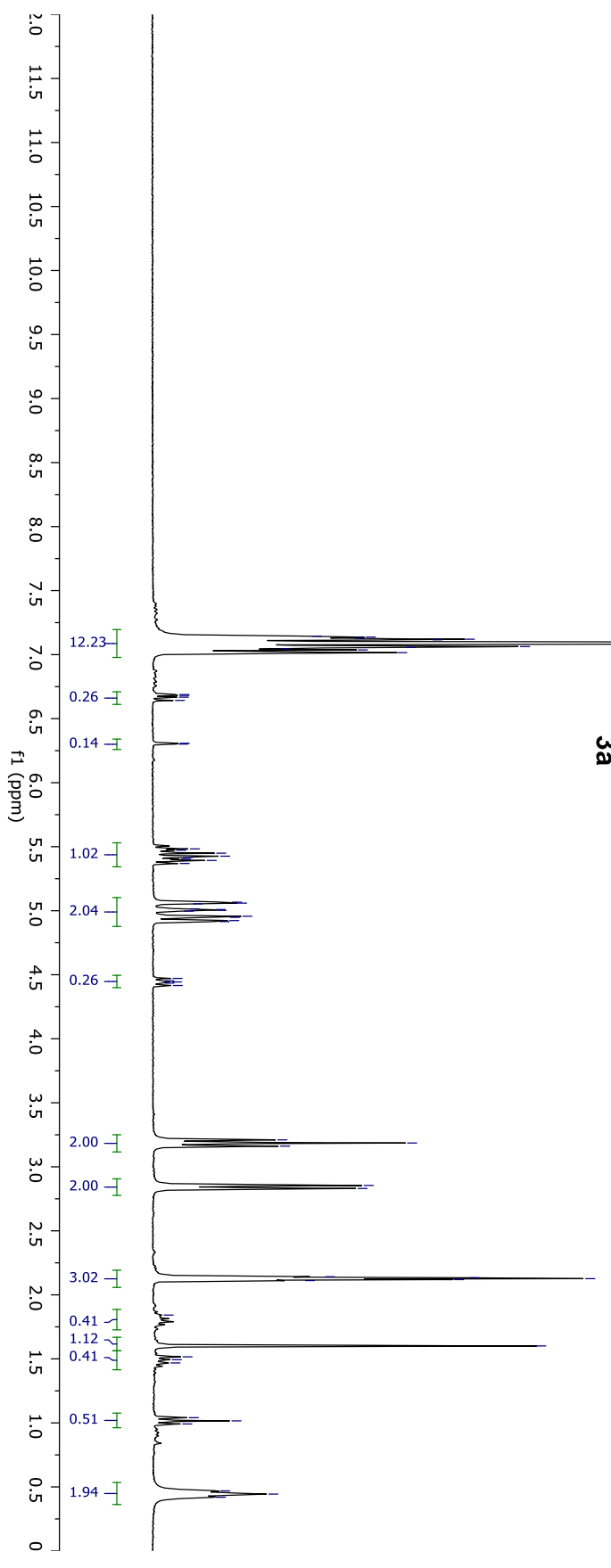

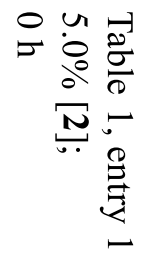
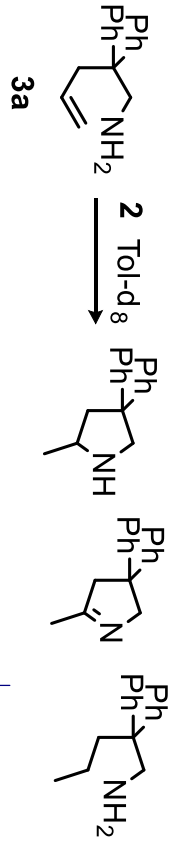

Figure S1. ${ }^{1} \mathrm{H}$ NMR spectrum for Table 1 , entry $1, \mathrm{t} 0$. 


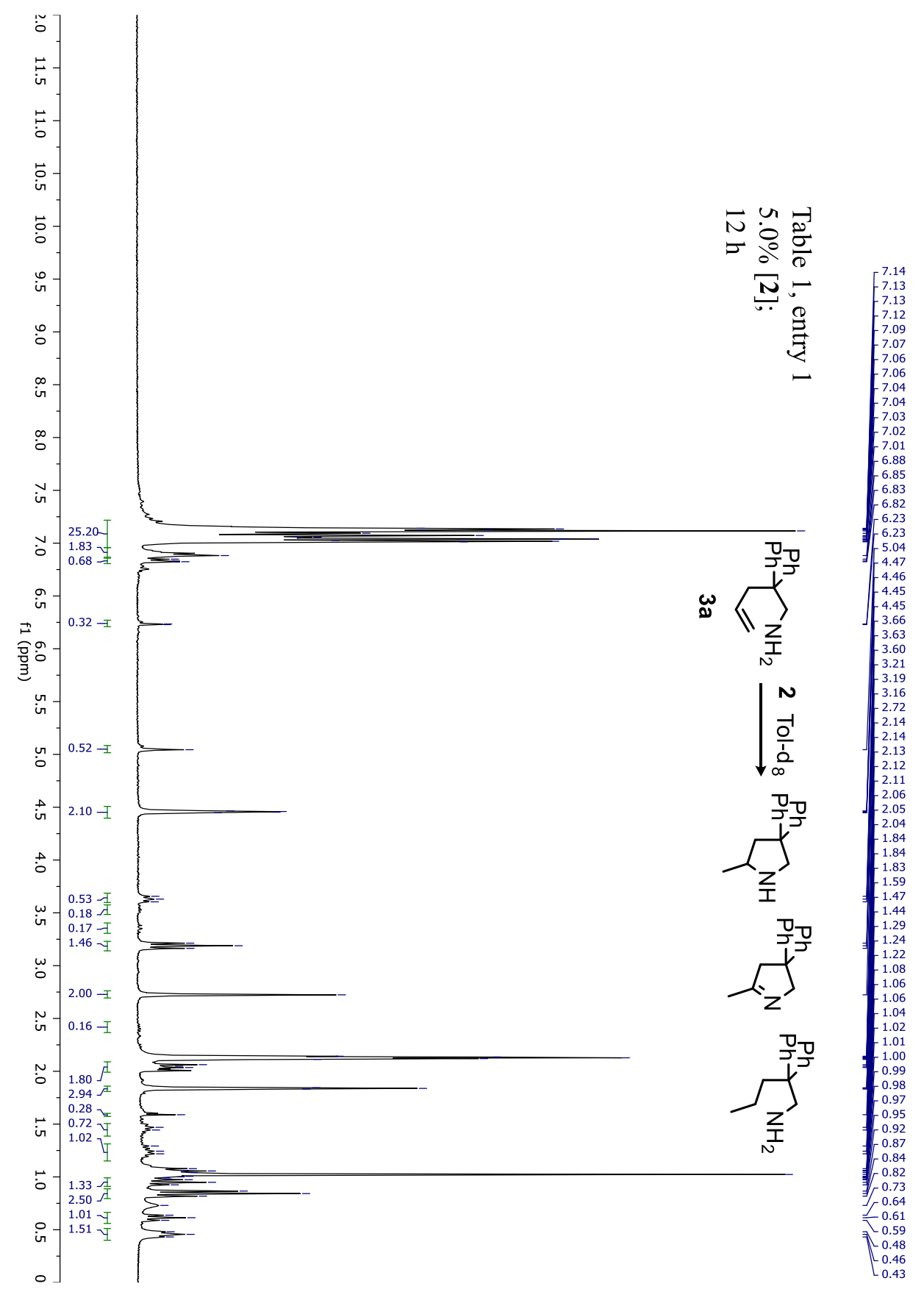

Figure S2. ${ }^{1} \mathrm{H}$ NMR spectrum for Table 1 , entry 1. 


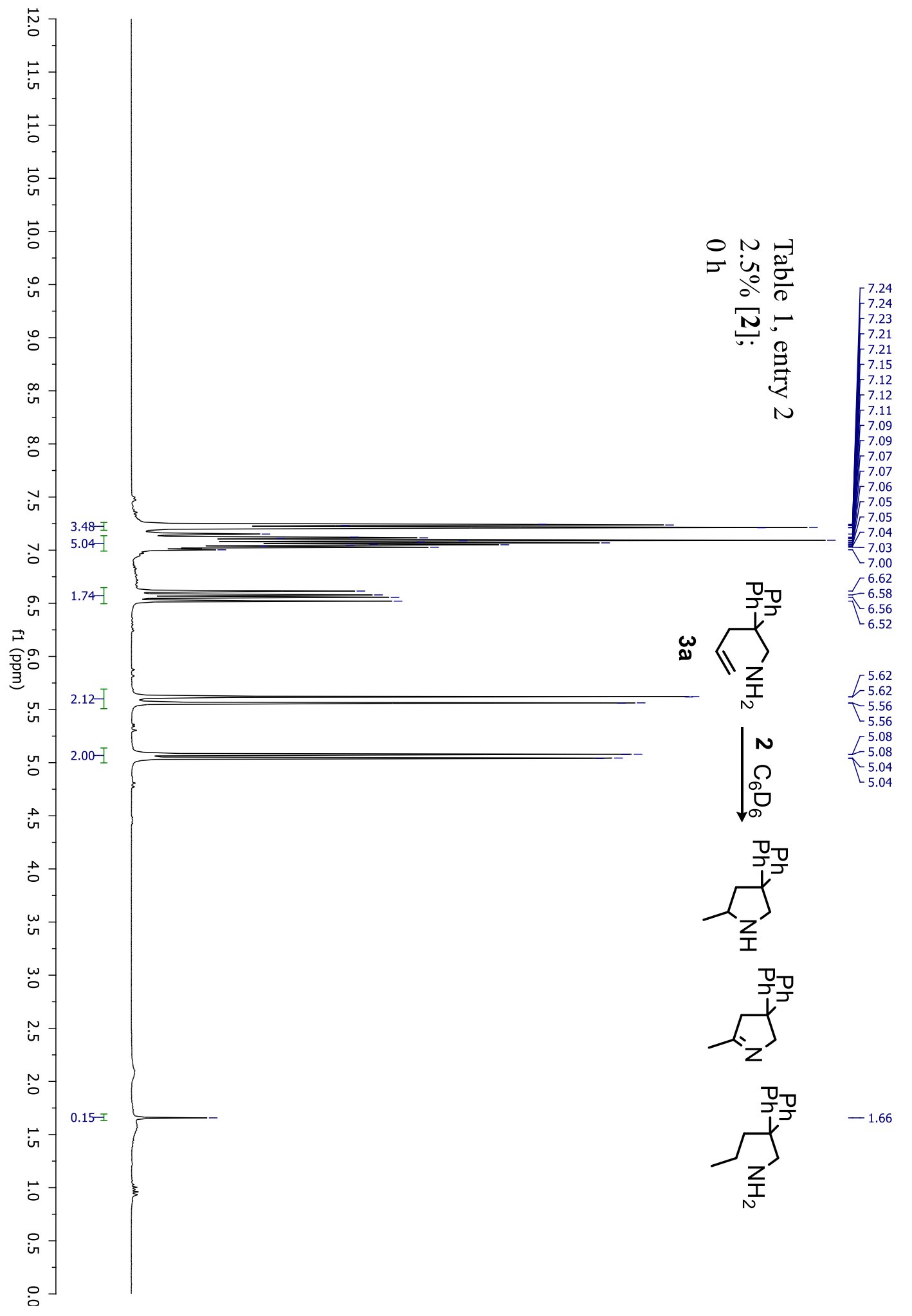

Figure S3. ${ }^{1} \mathrm{H}$ NMR spectrum for Table 1, entry 2 , t0. 


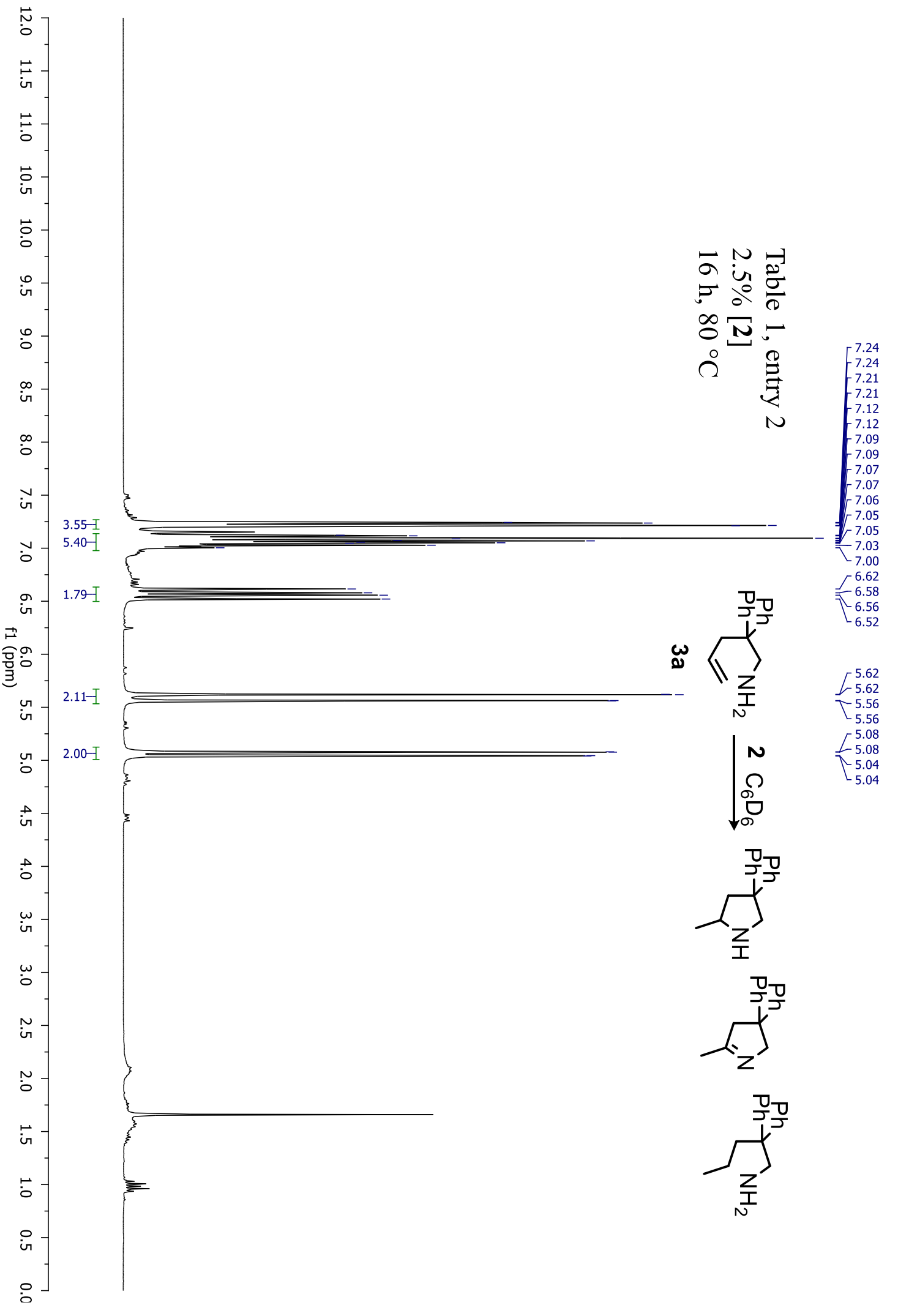

Figure S4. ${ }^{1} \mathrm{H}$ NMR spectrum for Table 1, entry 2. 


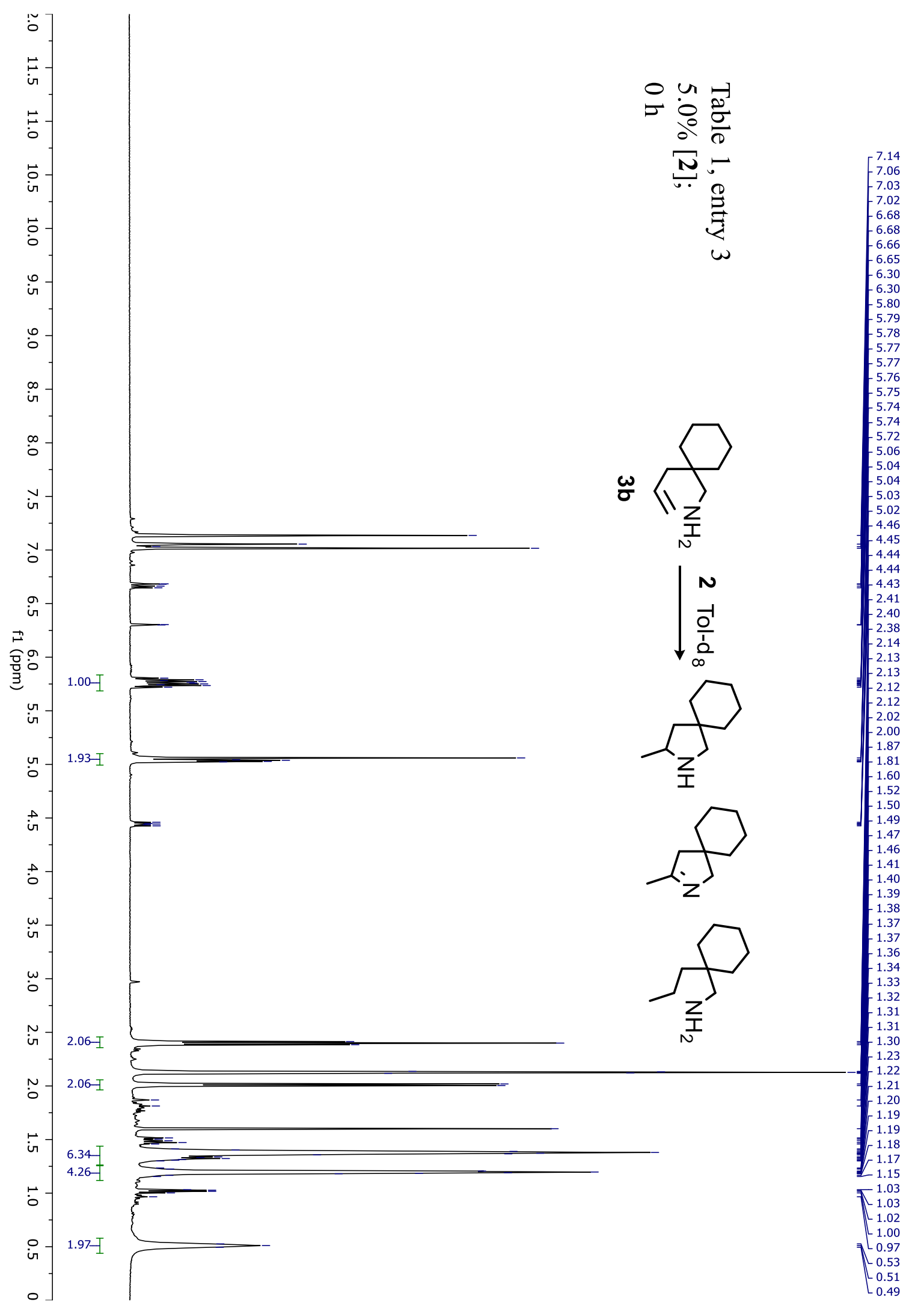

Figure S5. ${ }^{1} \mathrm{H}$ NMR spectrum for Table 1 , entry 3 , t0. 


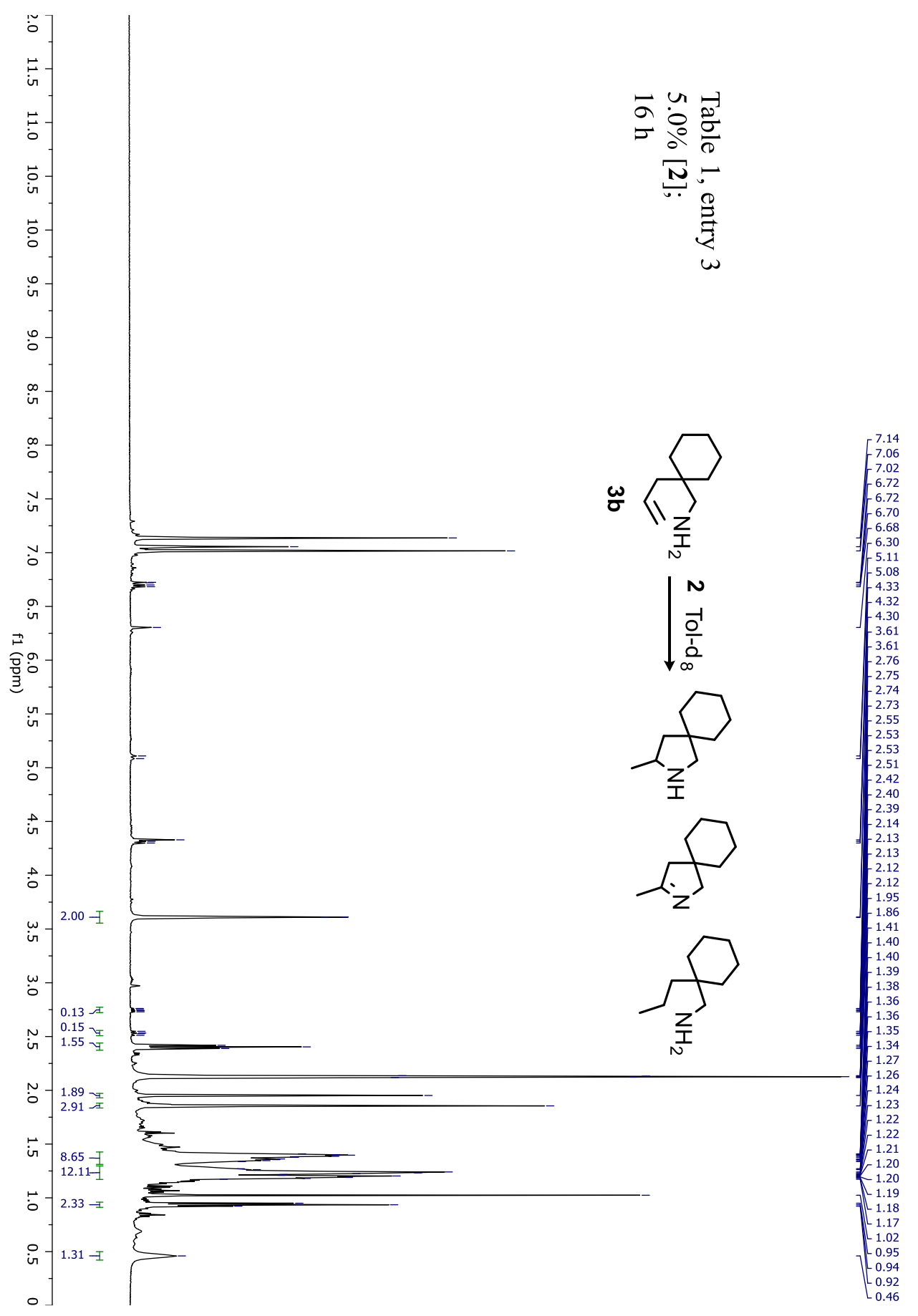

Figure S6. ${ }^{1} \mathrm{H}$ NMR spectrum for Table 1, entry 3. 


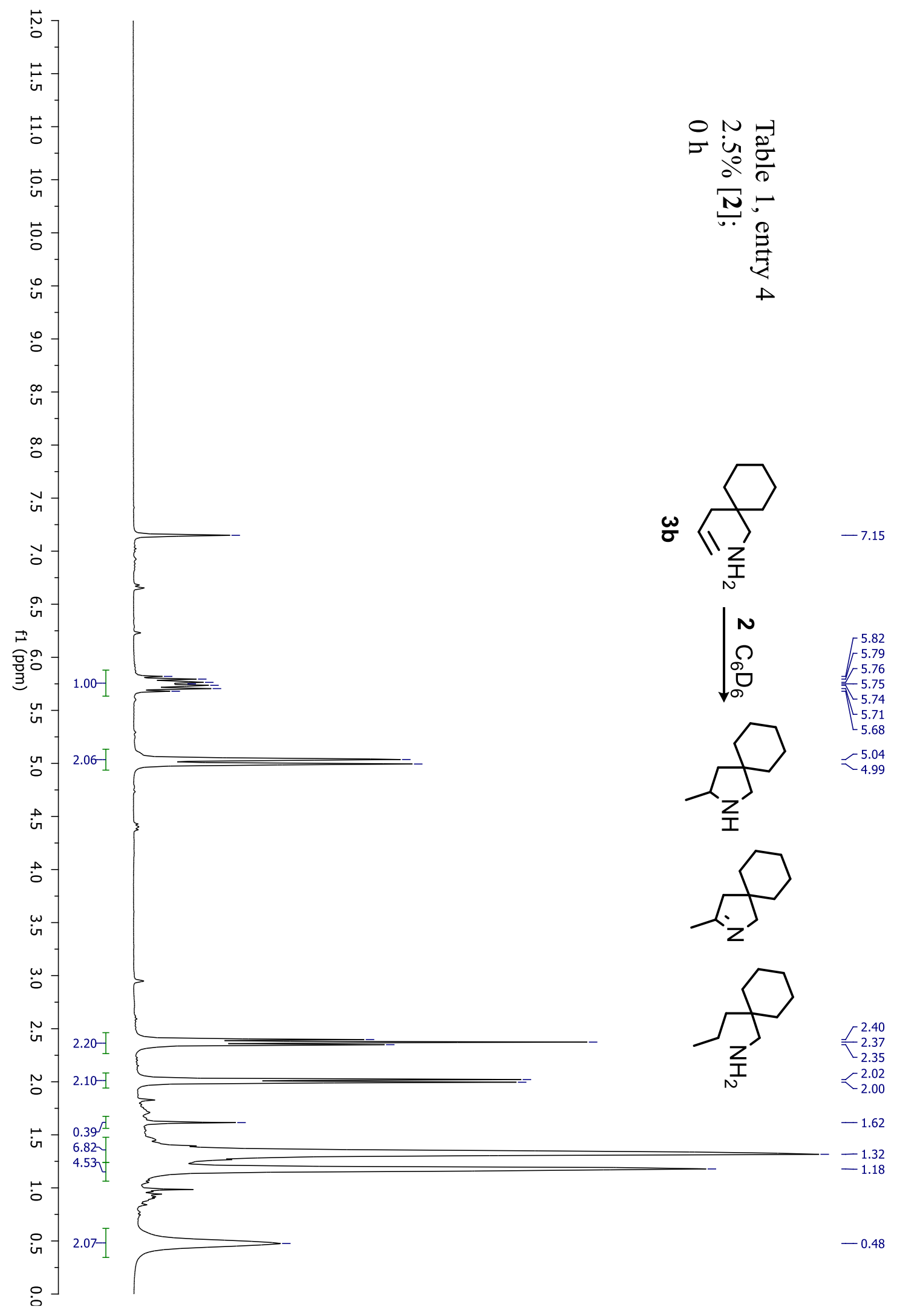

Figure S7. ${ }^{1} \mathrm{H}$ NMR spectrum for Table 1 , entry 4 , t0. 


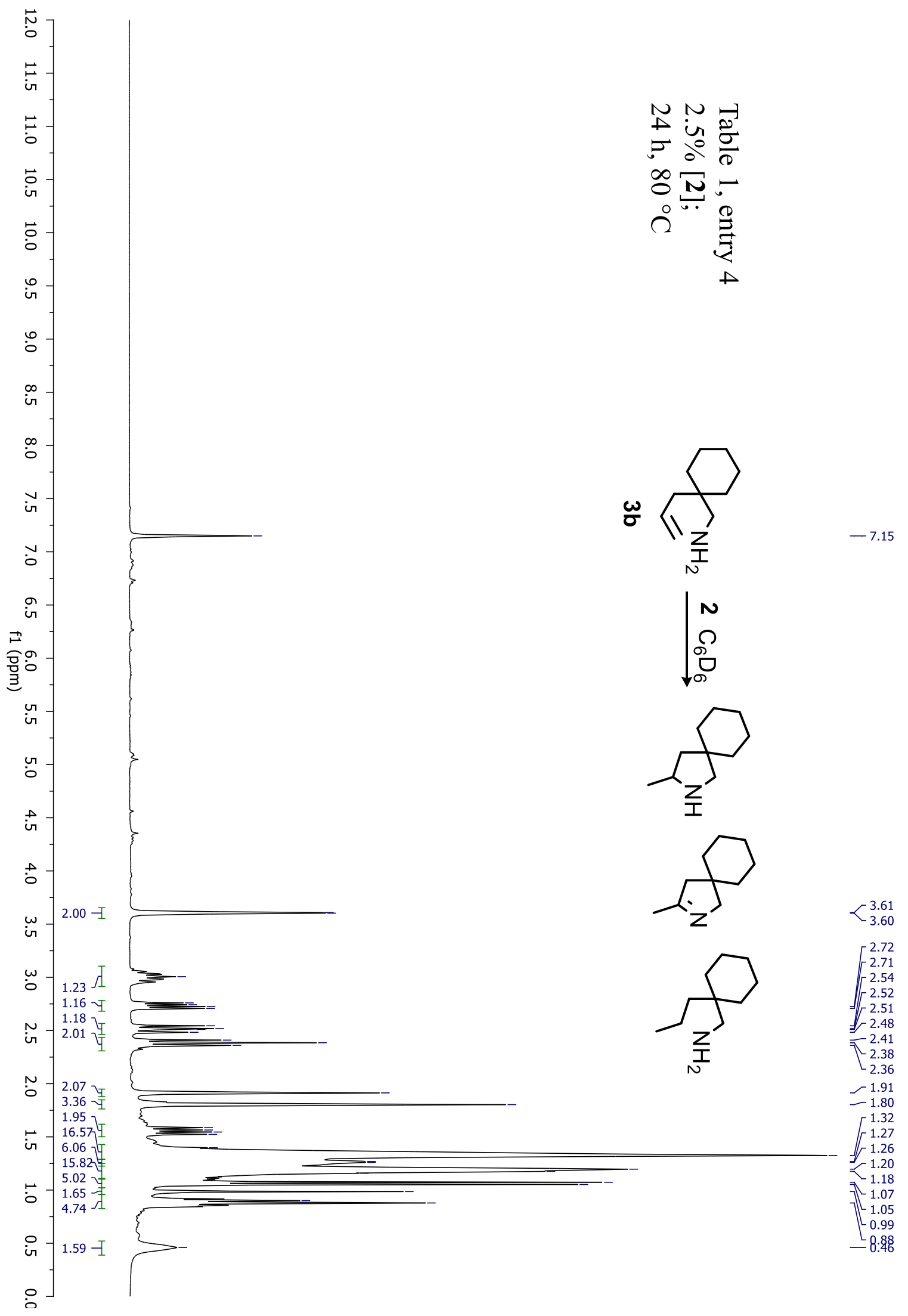

Figure S8. ${ }^{1} \mathrm{H}$ NMR spectrum for Table 1, entry 4. 


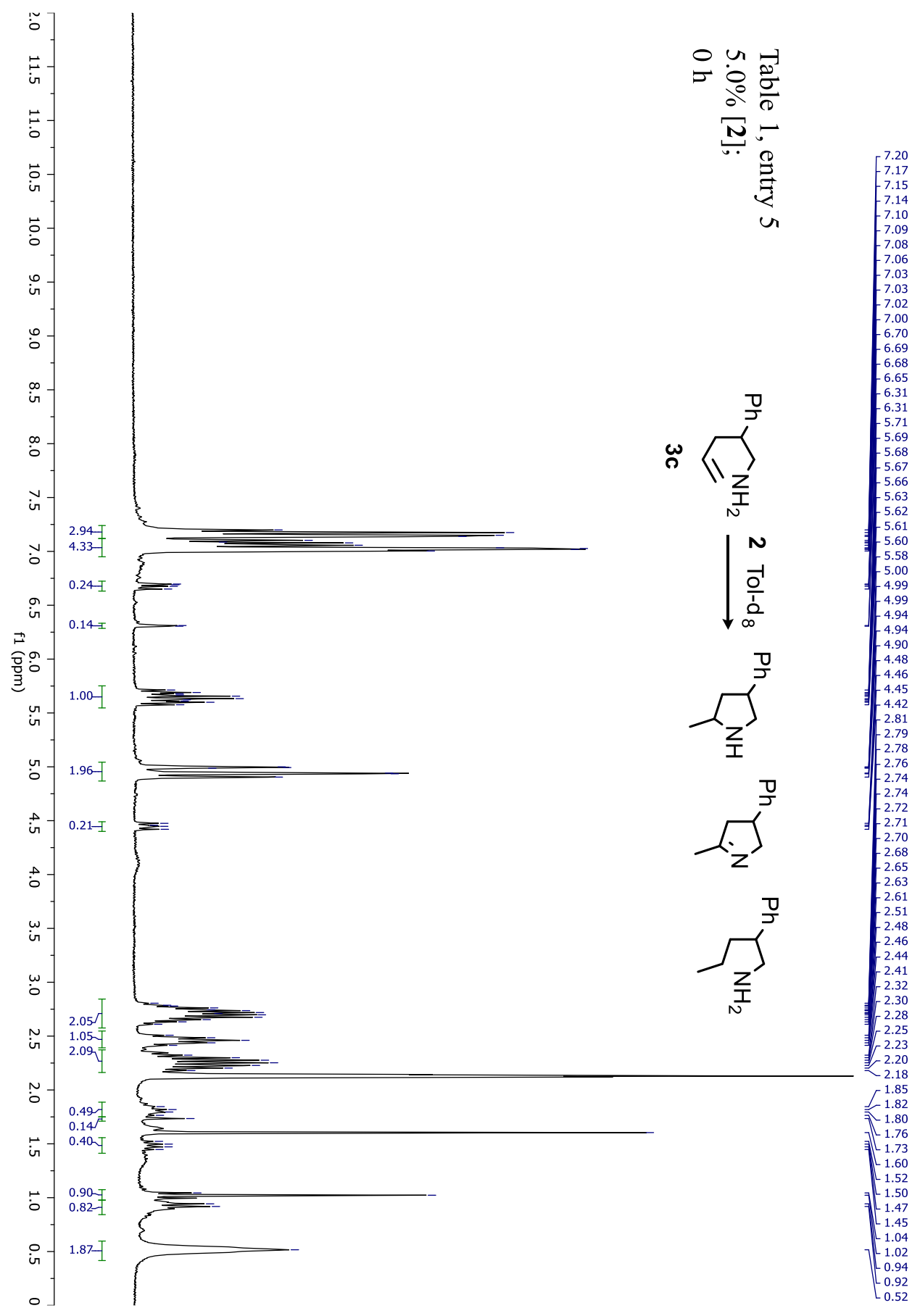

Figure S9. ${ }^{1} \mathrm{H}$ NMR spectrum for Table 1, entry 5, t0. 


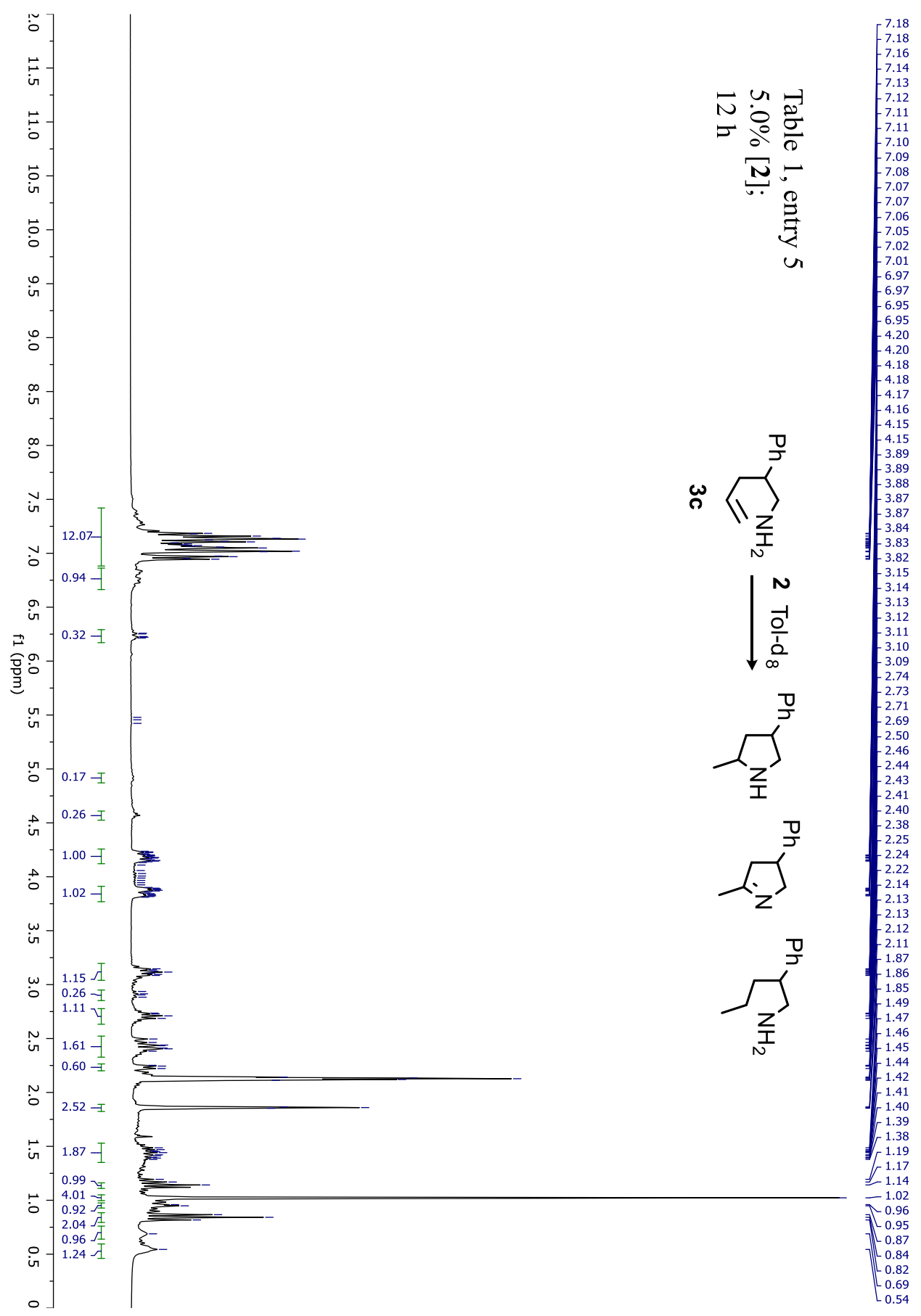

Figure S10. ${ }^{1} \mathrm{H}$ NMR spectrum for Table 1, entry 5. 


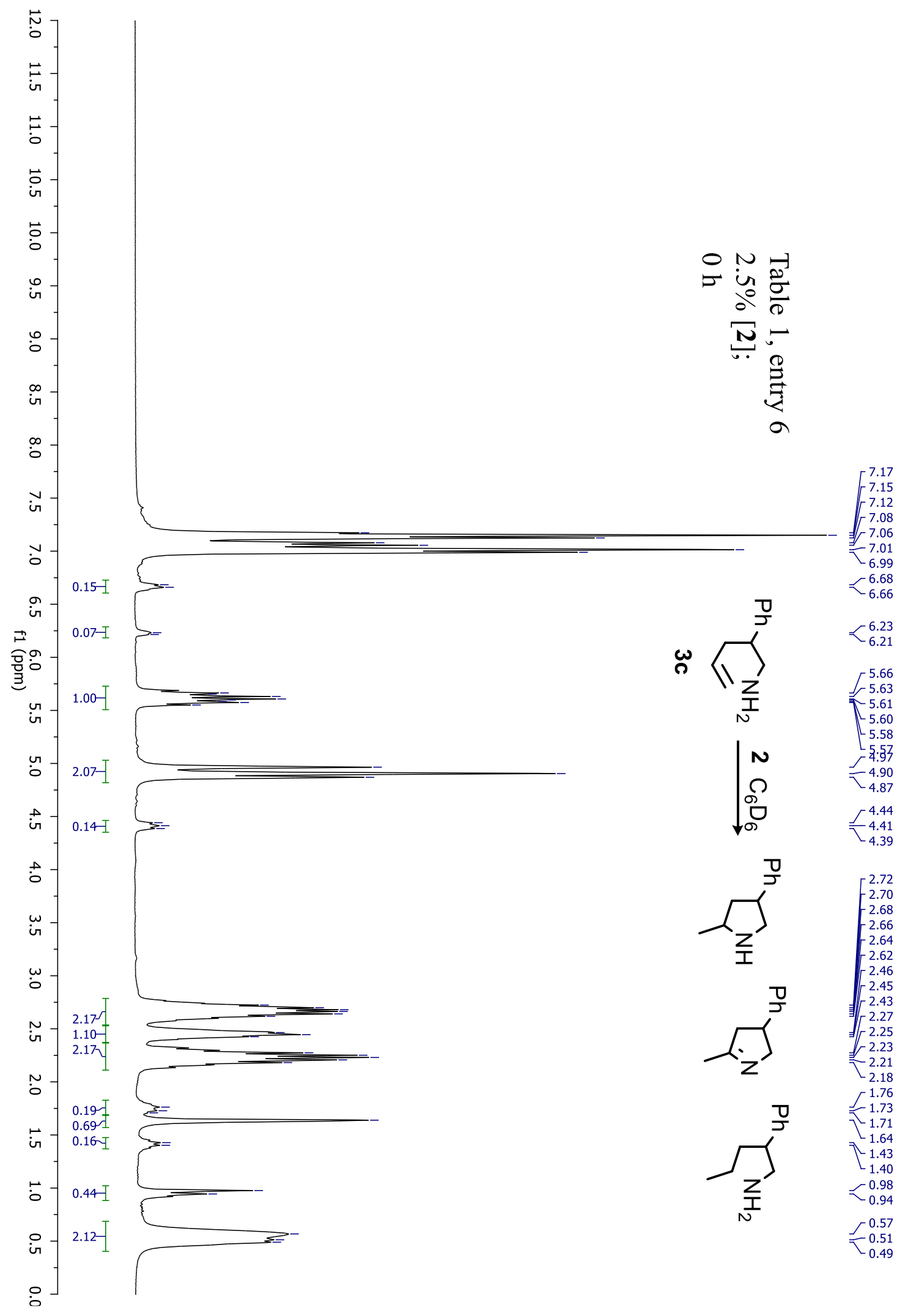

Figure S11. ${ }^{1} \mathrm{H}$ NMR spectrum for Table 1 , entry 6, t0. 


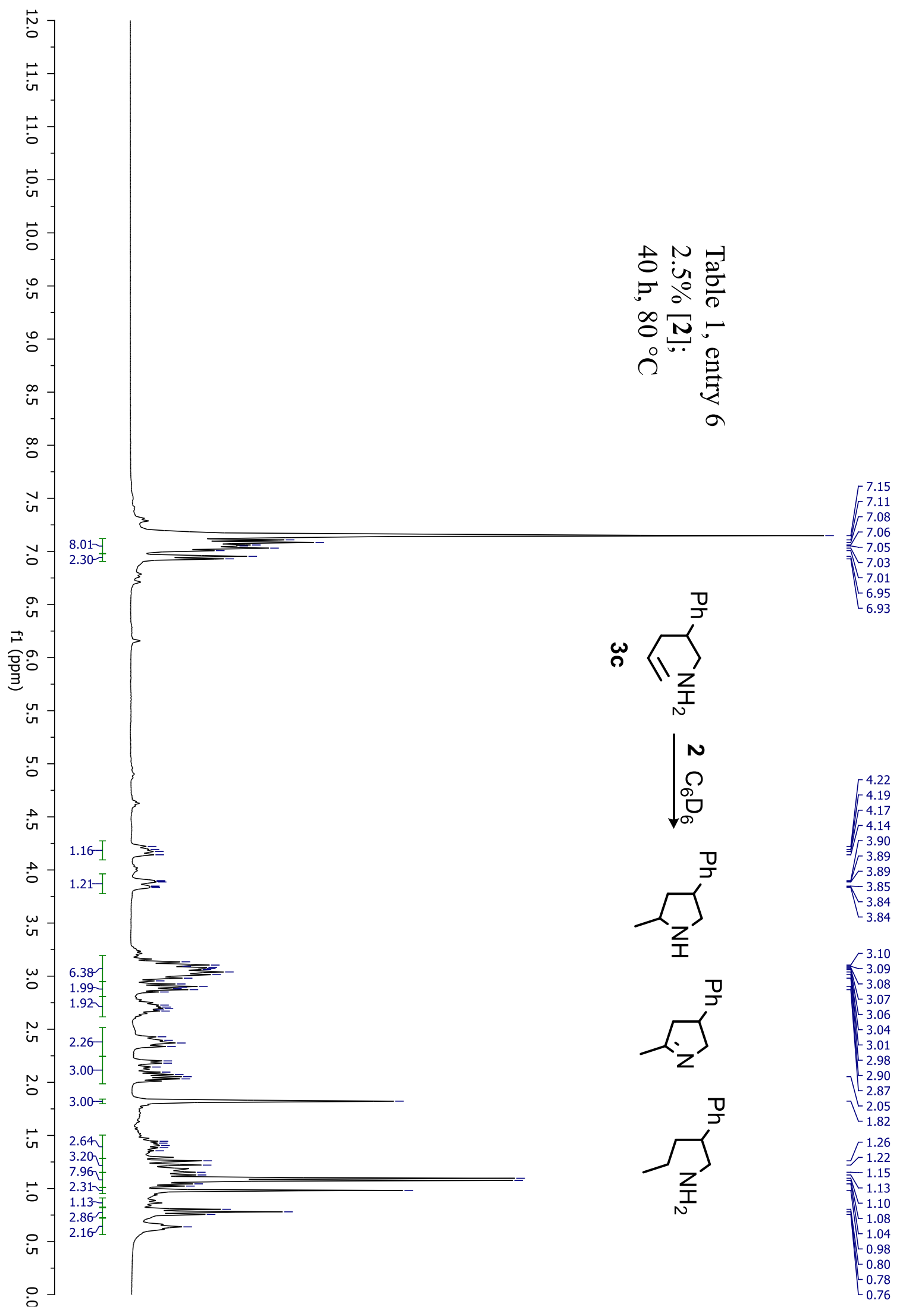

Figure S12. ${ }^{1} \mathrm{H}$ NMR spectrum for Table 1, entry 6. 


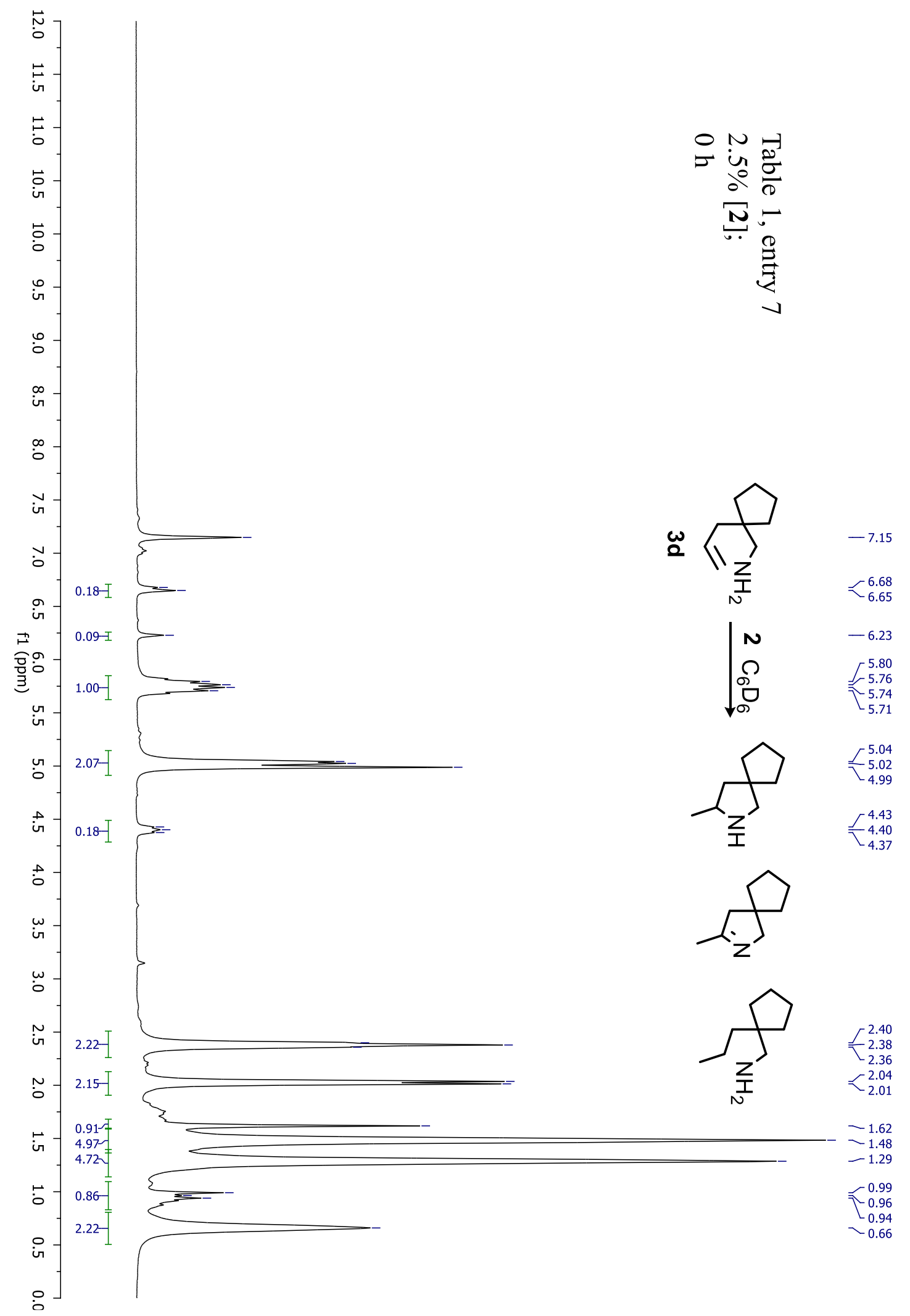

Figure S13. ${ }^{1} \mathrm{H}$ NMR spectrum for Table 1, entry 7, t0. 


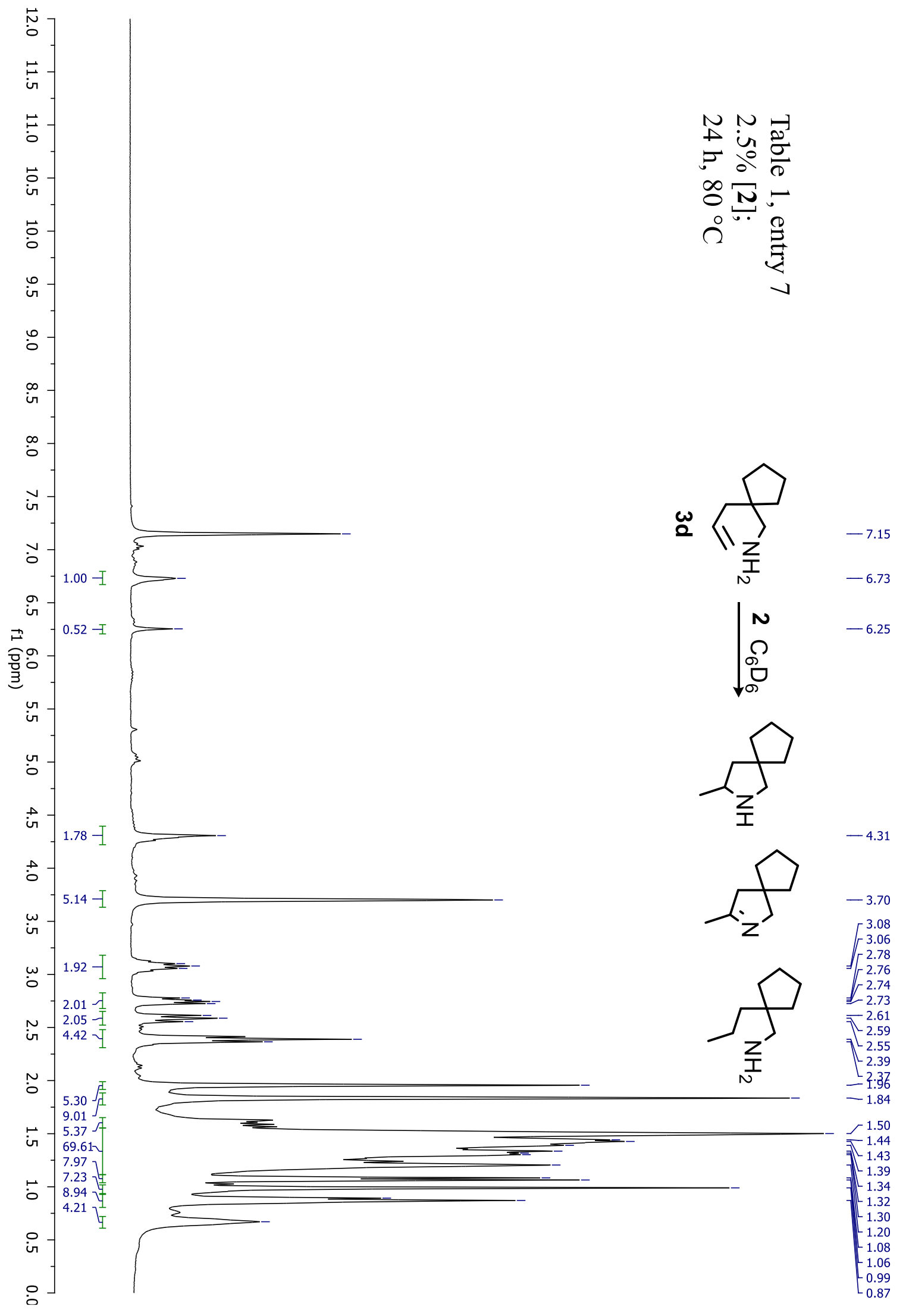

Figure S14. ${ }^{1} \mathrm{H}$ NMR spectrum for Table 1, entry 7. 


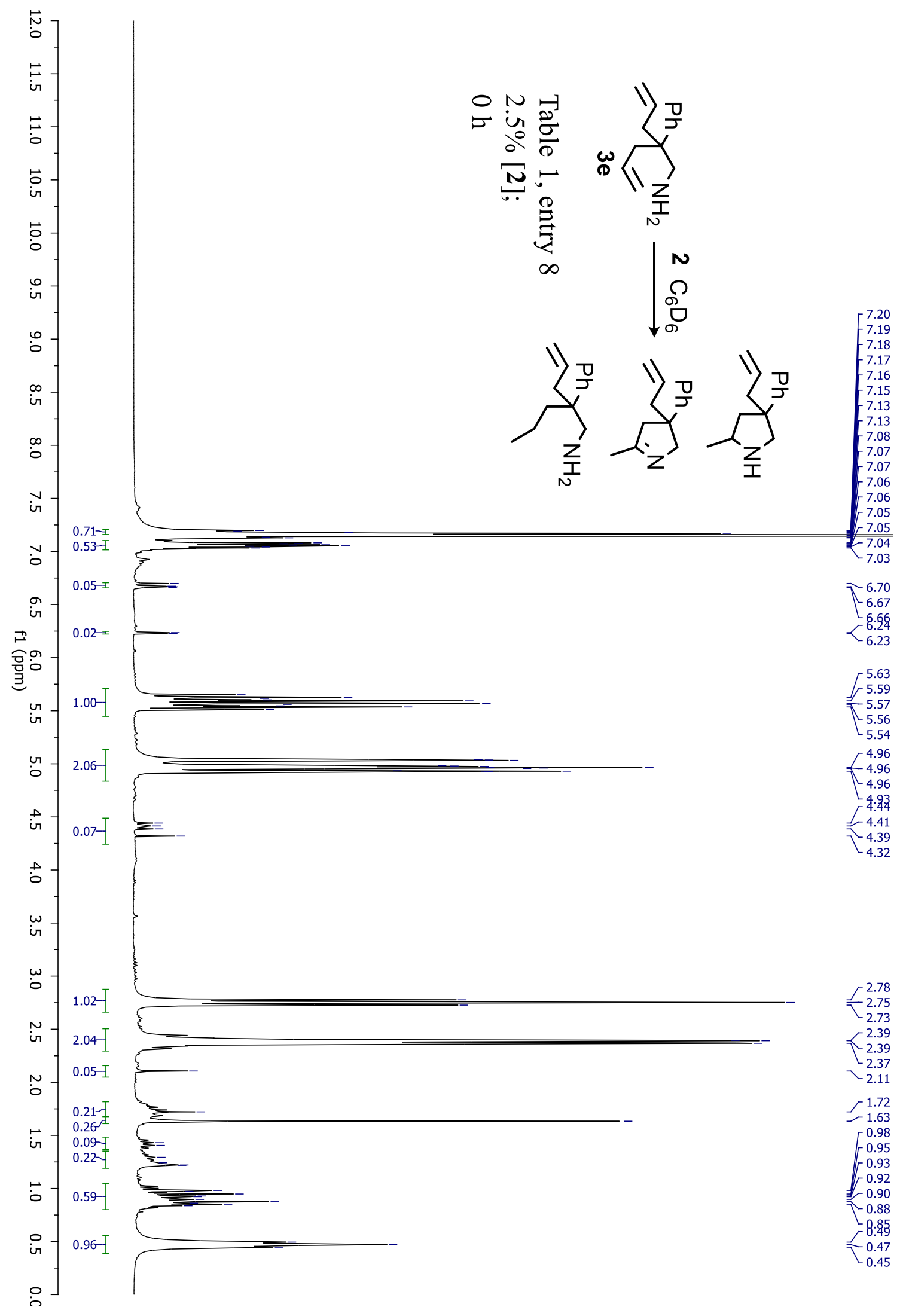

Figure S15. ${ }^{1} \mathrm{H}$ NMR spectrum for Table 1 , entry 8 , to. 


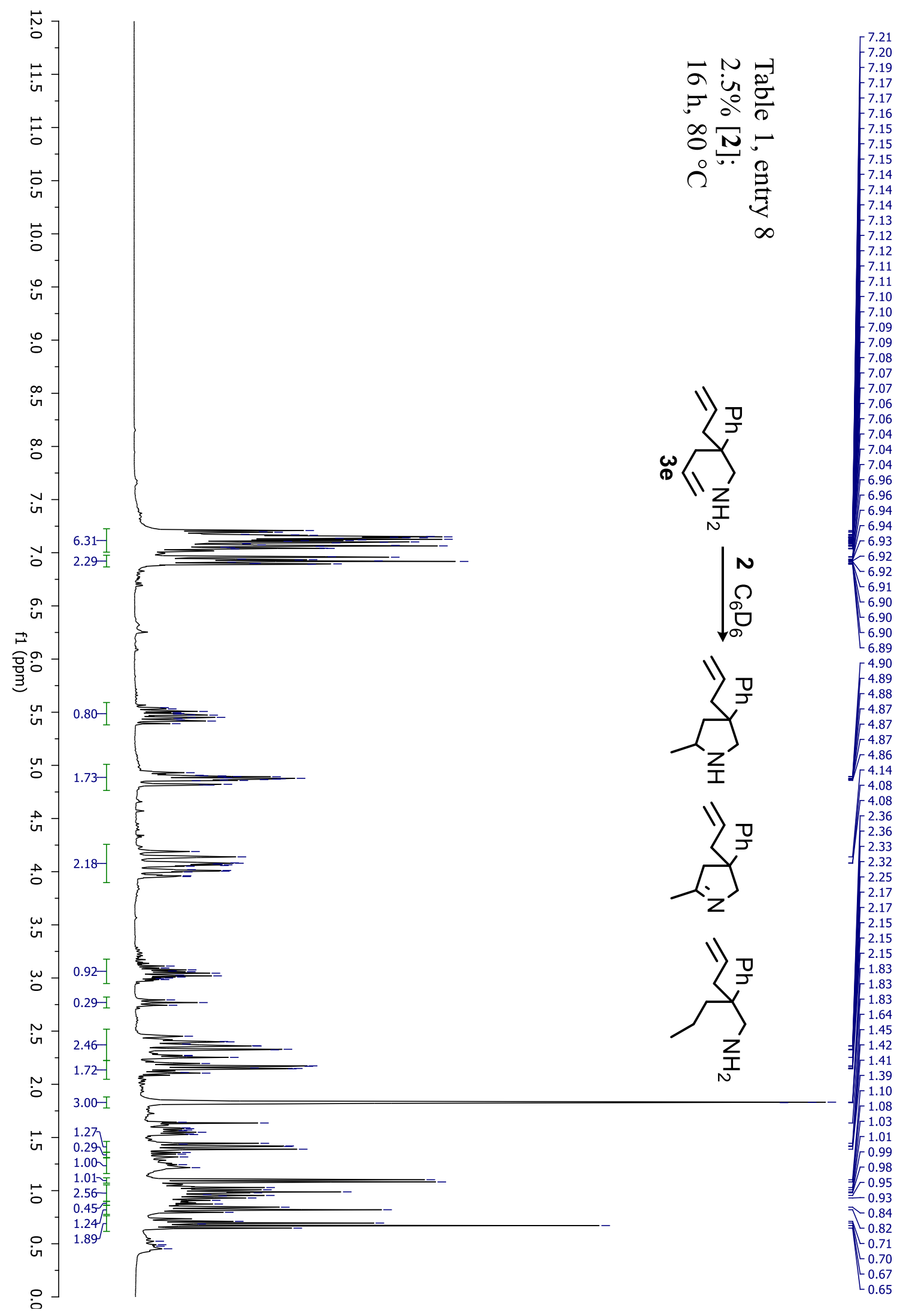

Figure S16. ${ }^{1} \mathrm{H}$ NMR spectrum for Table 1, entry 8. 

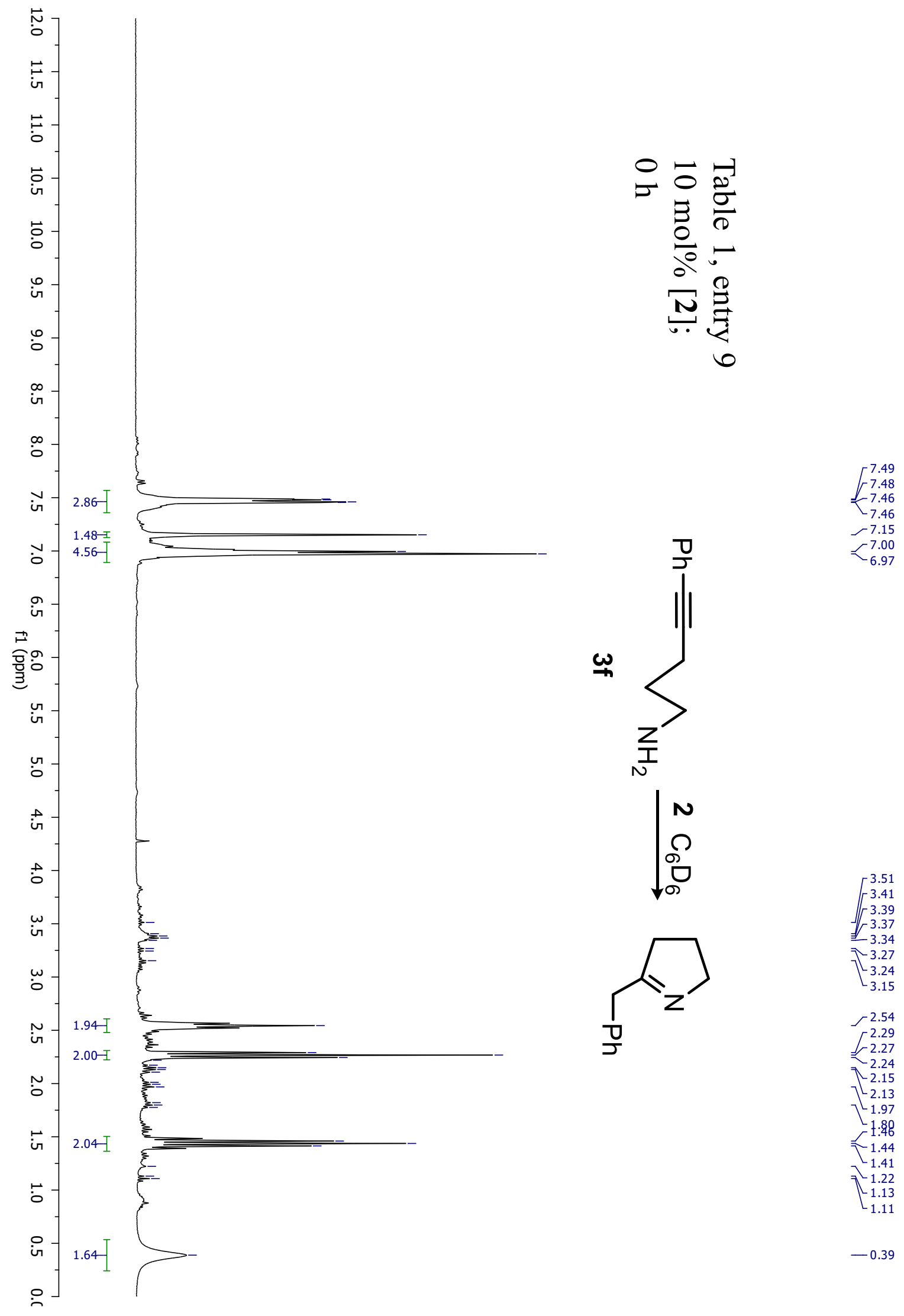

2.54

2.29
-2.27

2.24

2.15

]$_{1.97}^{2.13}$

1.80
-1.44

$\tau_{1.41}^{1.44}$

- 1.22

$1_{1.13}^{-1.22}$

$-0.39$

Figure S17. ${ }^{1} \mathrm{H}$ NMR spectrum for Table 1, entry 9, t0. 


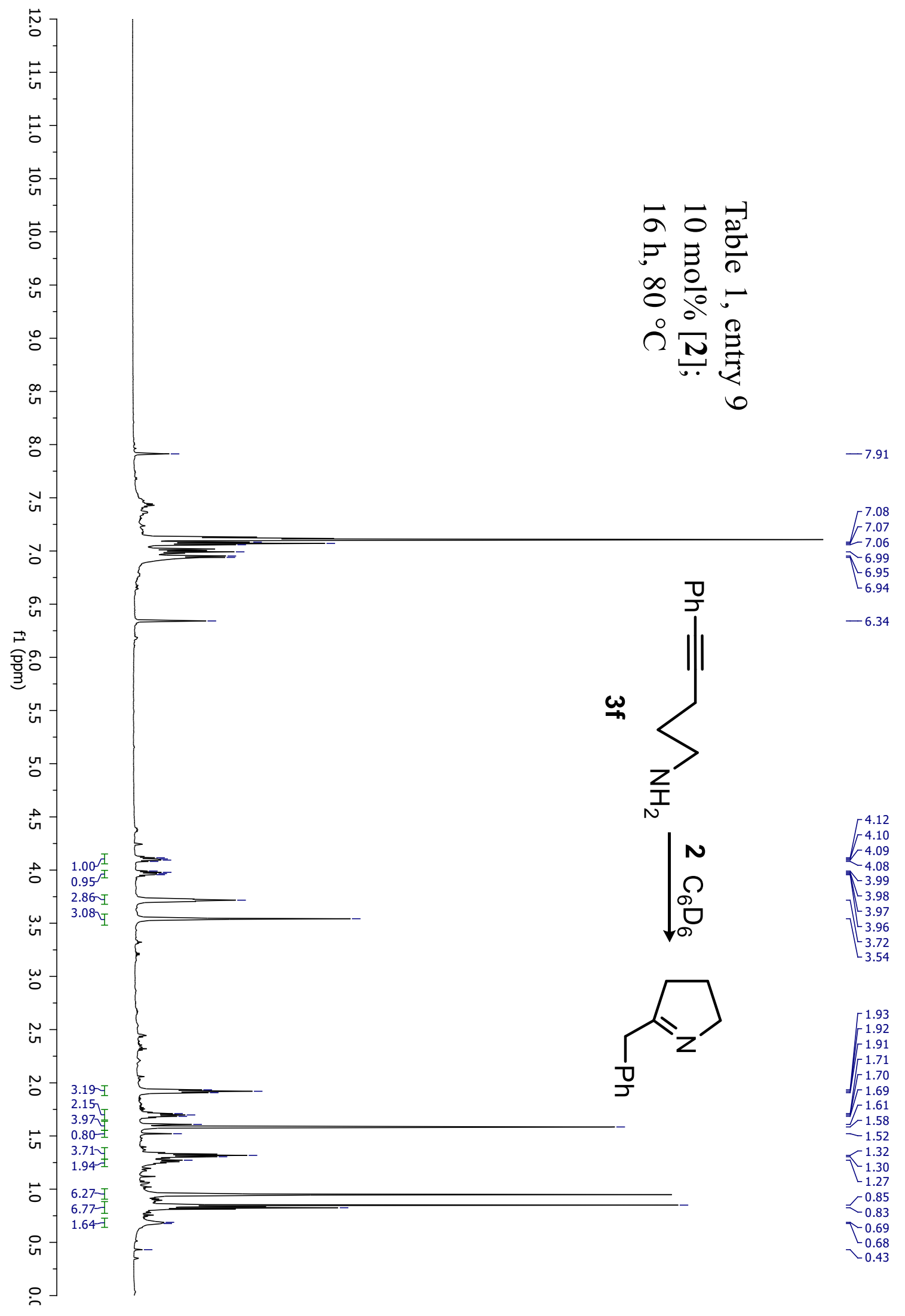

Figure S18. ${ }^{1} \mathrm{H}$ NMR spectrum for Table 1, entry 9. 


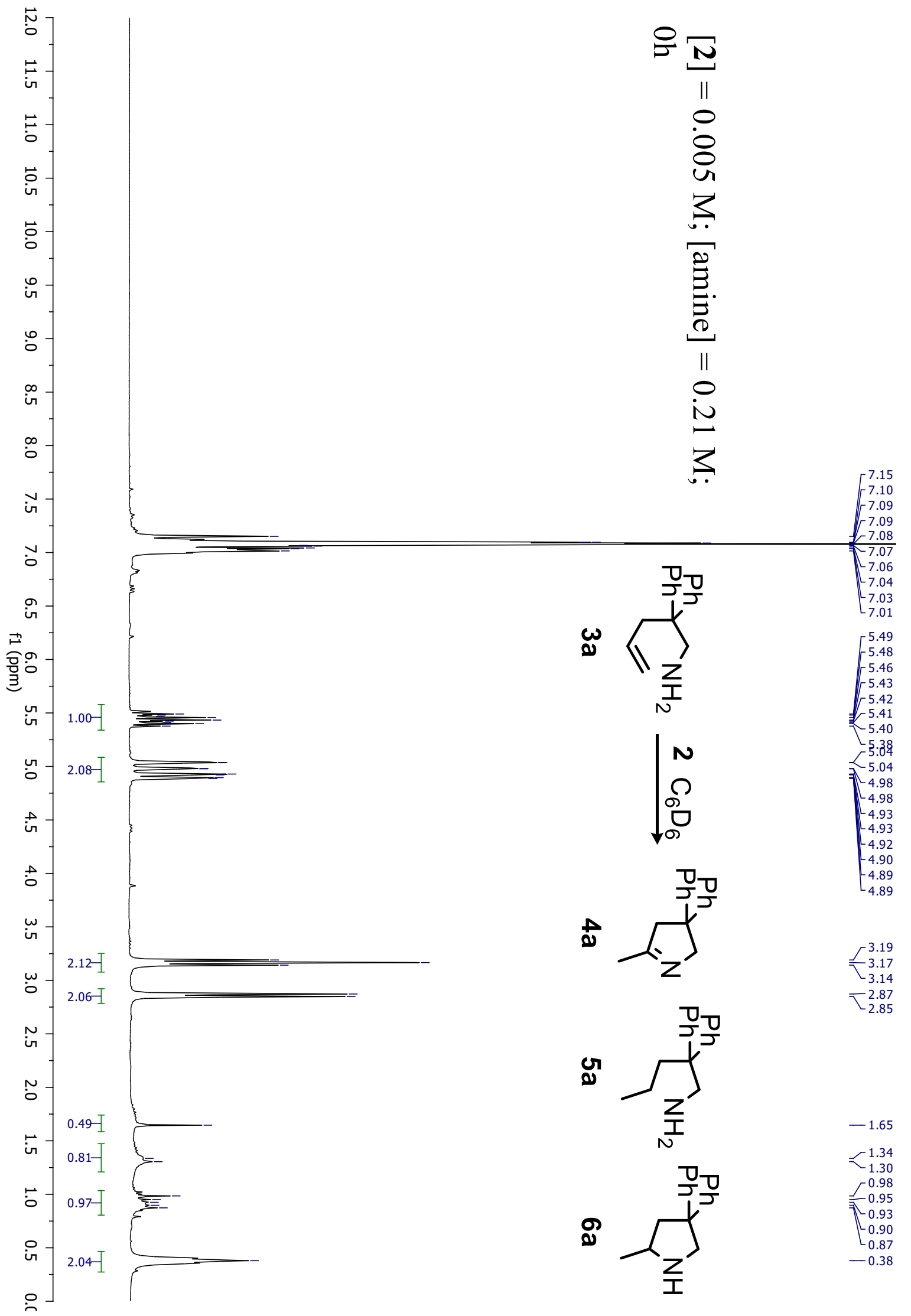

Figure S19. ${ }^{1} \mathrm{H}$ NMR spectrum for kinetic study, t0. 


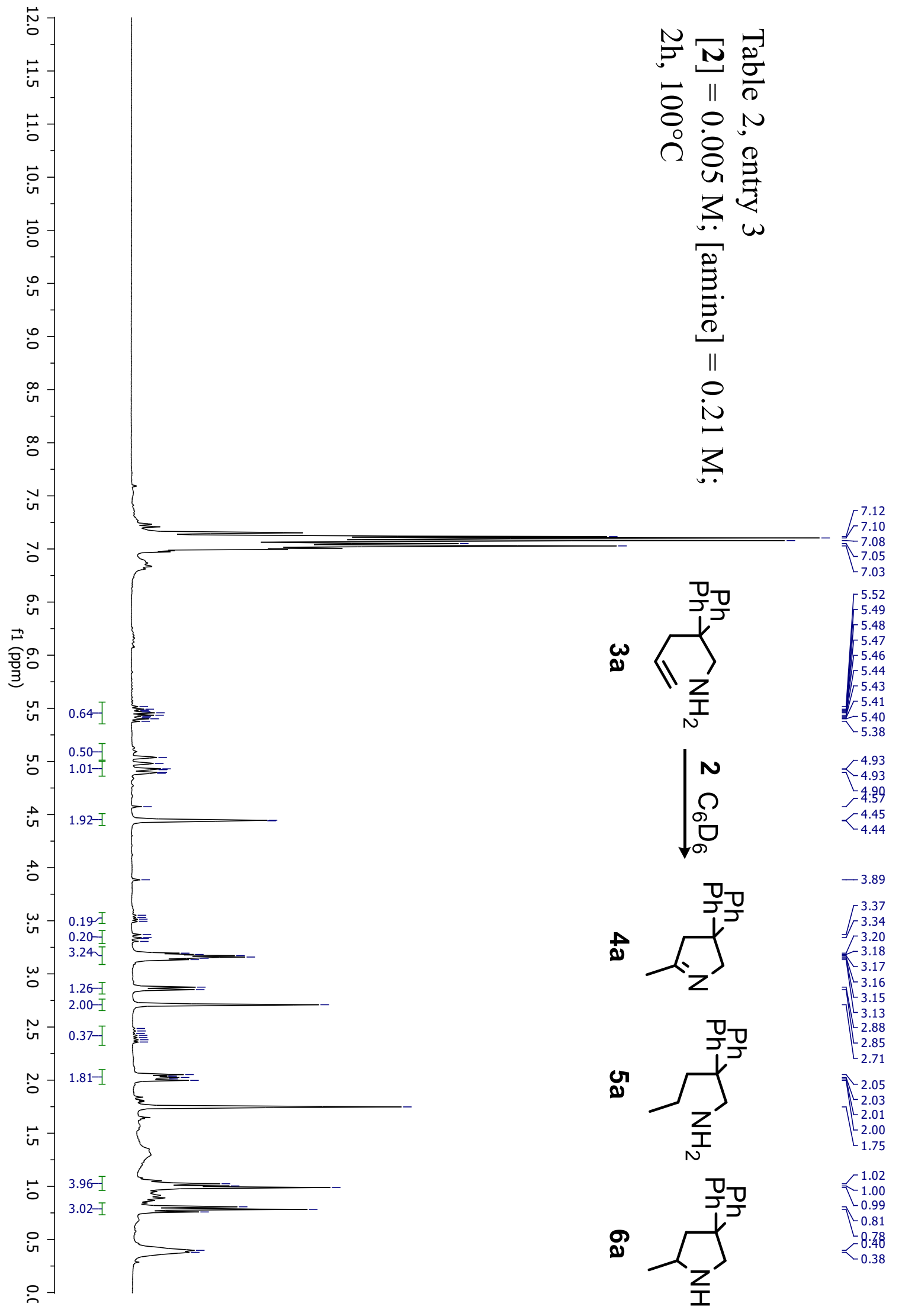

Figure S20. ${ }^{1} \mathrm{H}$ NMR spectrum for Table 2, entry 3. 


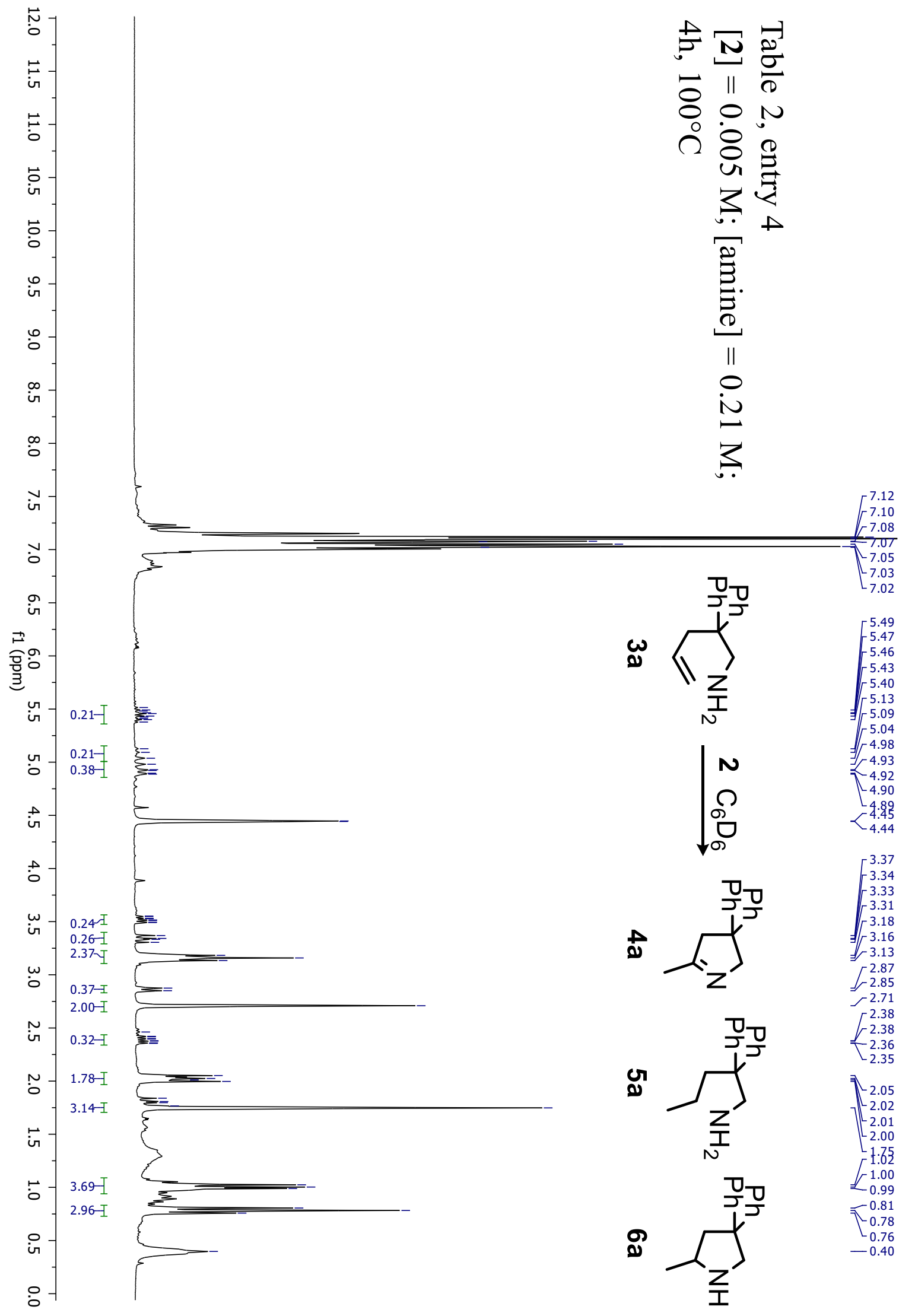

Figure S21. ${ }^{1} \mathrm{H}$ NMR spectrum for Table 2, entry 4. 


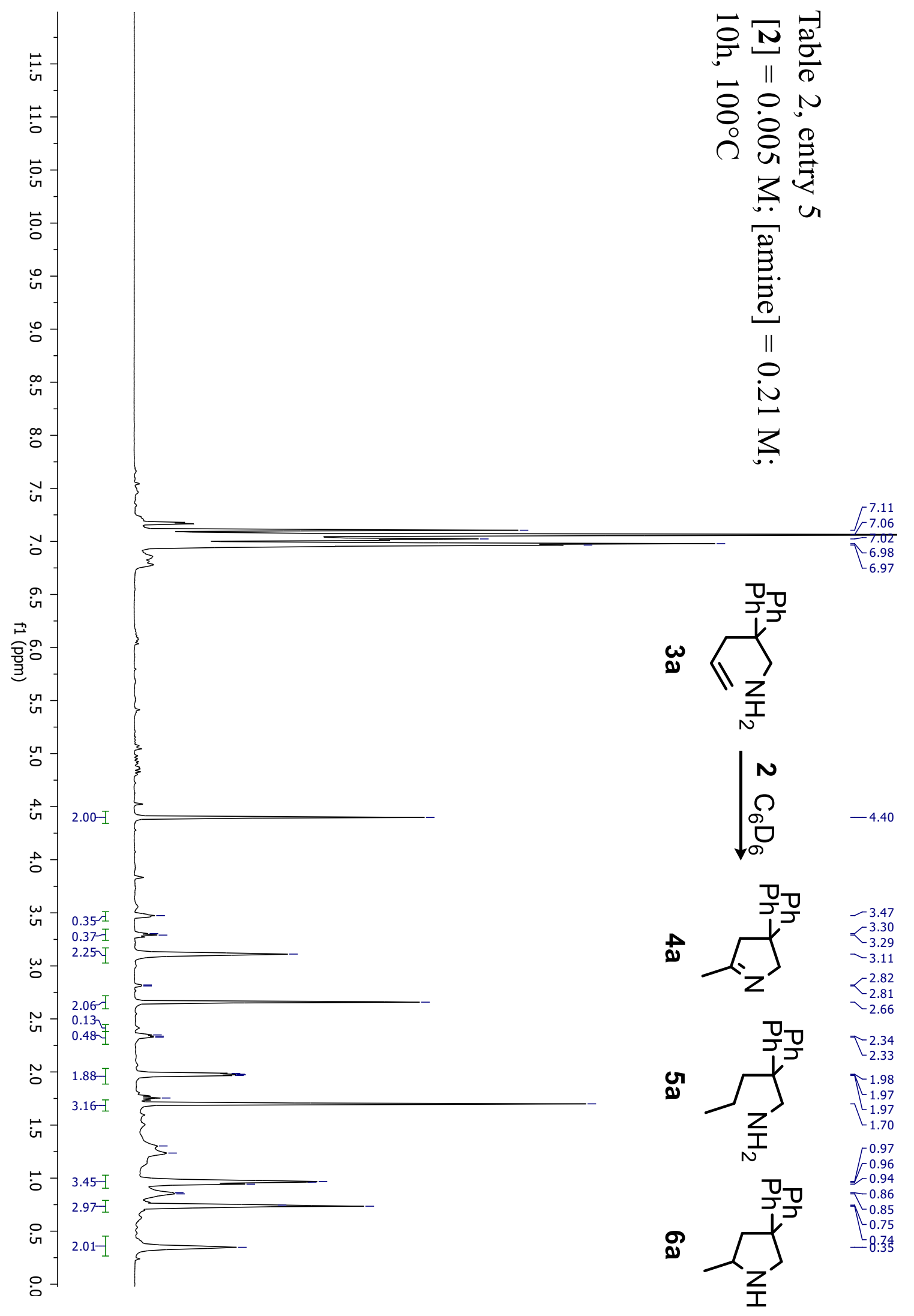

Figure S22. ${ }^{1} \mathrm{H}$ NMR spectrum for Table 2, entry 5. 


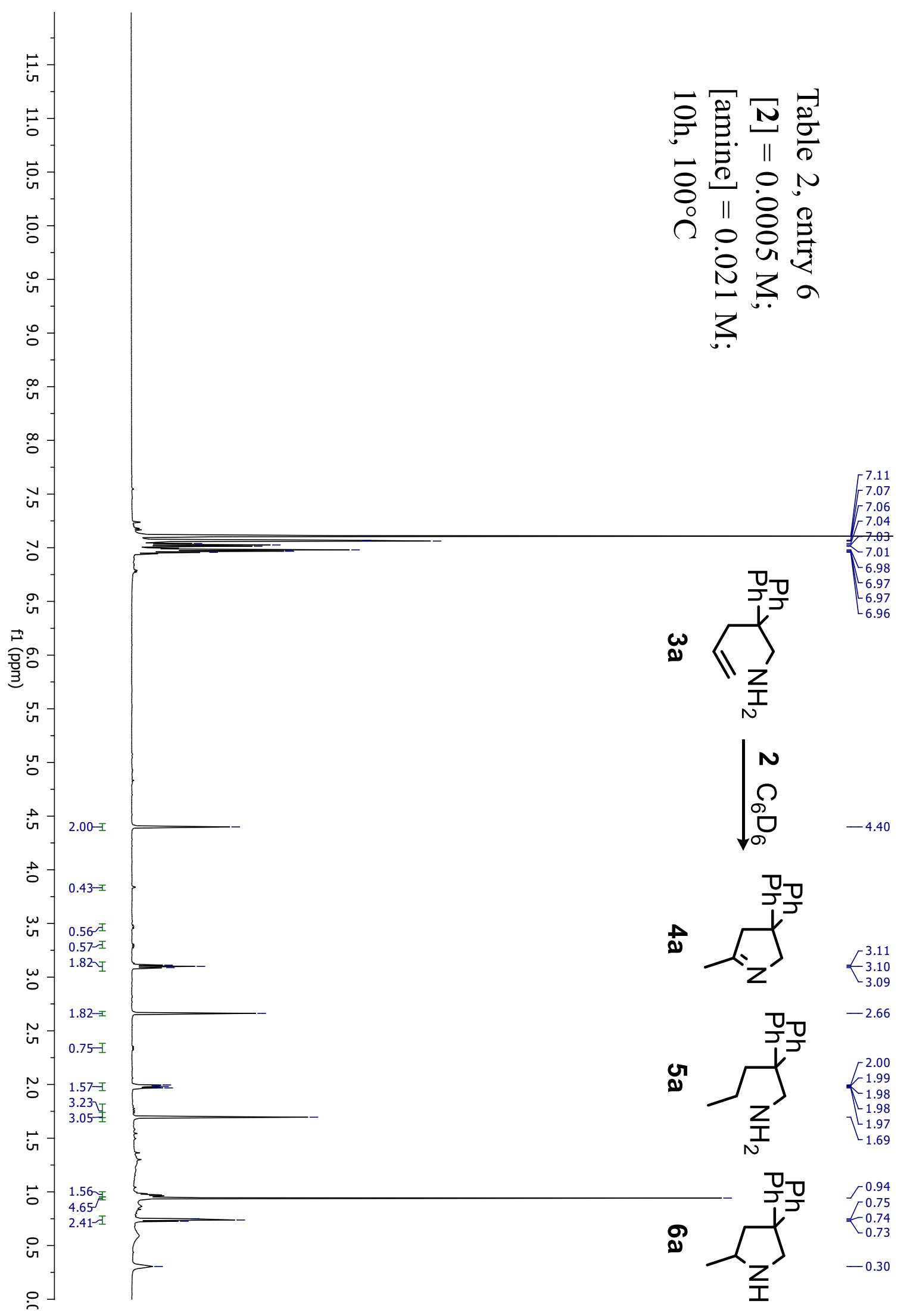

Figure S23. ${ }^{1} \mathrm{H}$ NMR spectrum for Table 2, entry 6. 


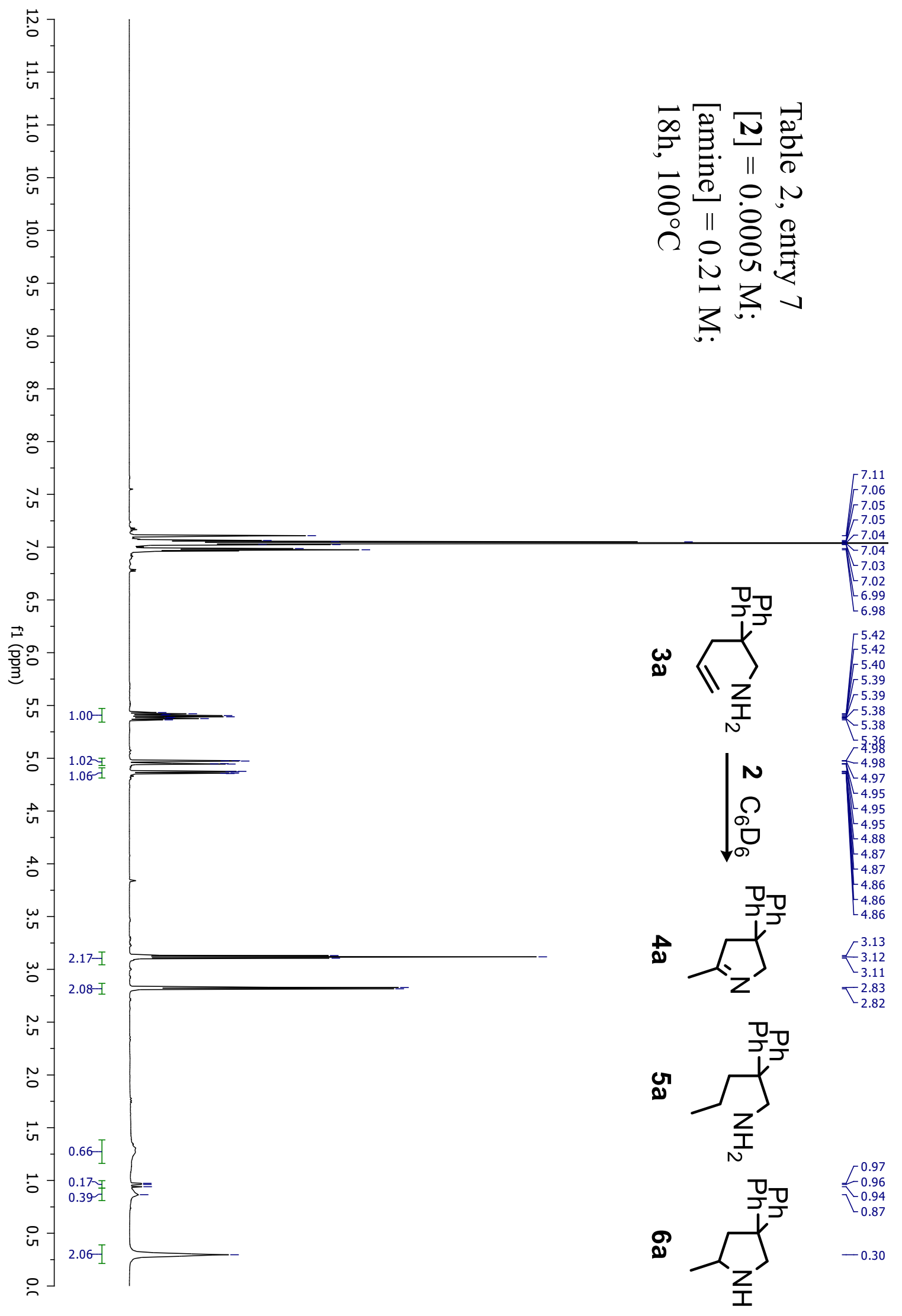

Figure S24. ${ }^{1} \mathrm{H}$ NMR spectrum for Table 2, entry 7. 


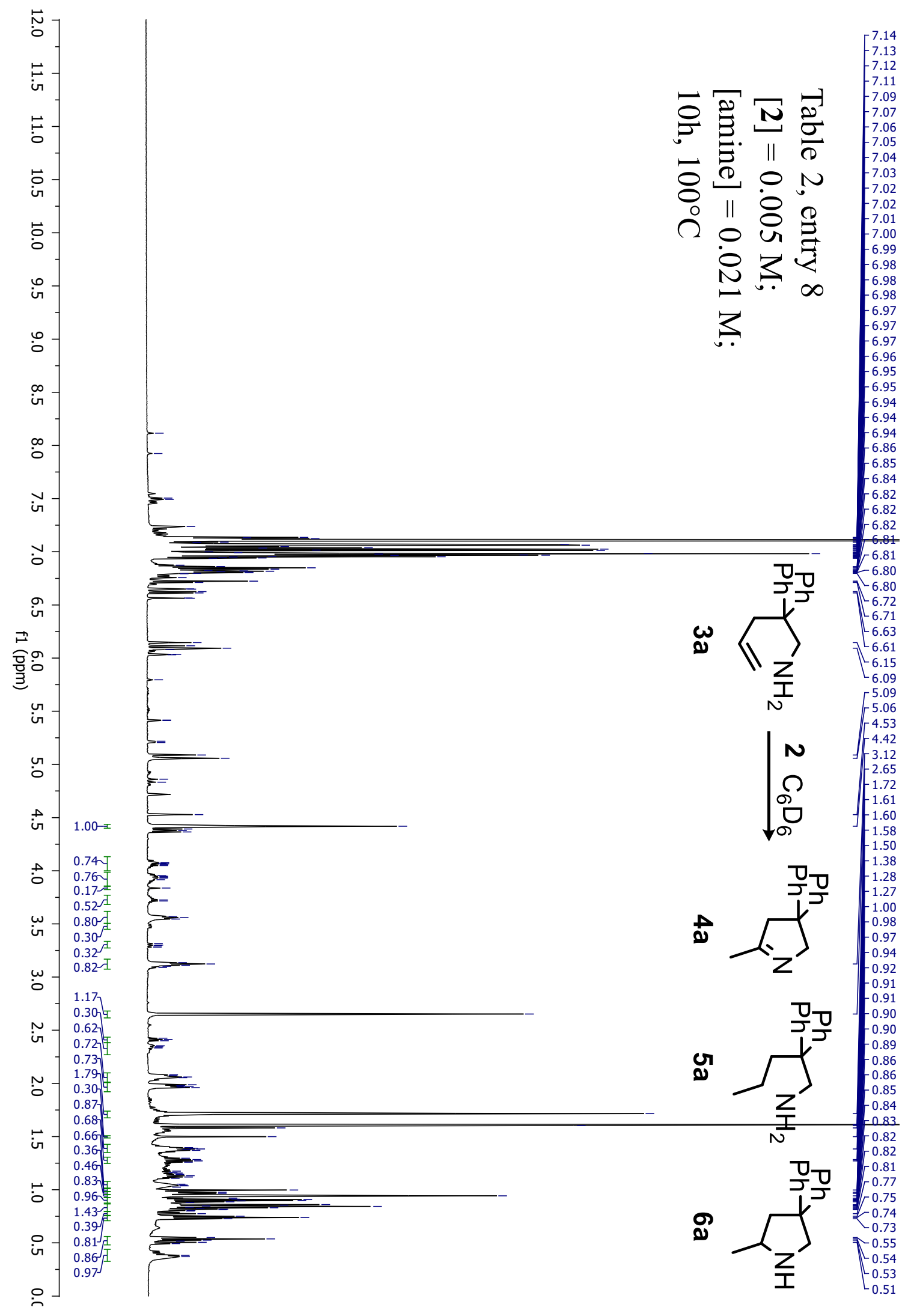

Figure S25. ${ }^{1} \mathrm{H}$ NMR spectrum for Table 2, entry 8. 


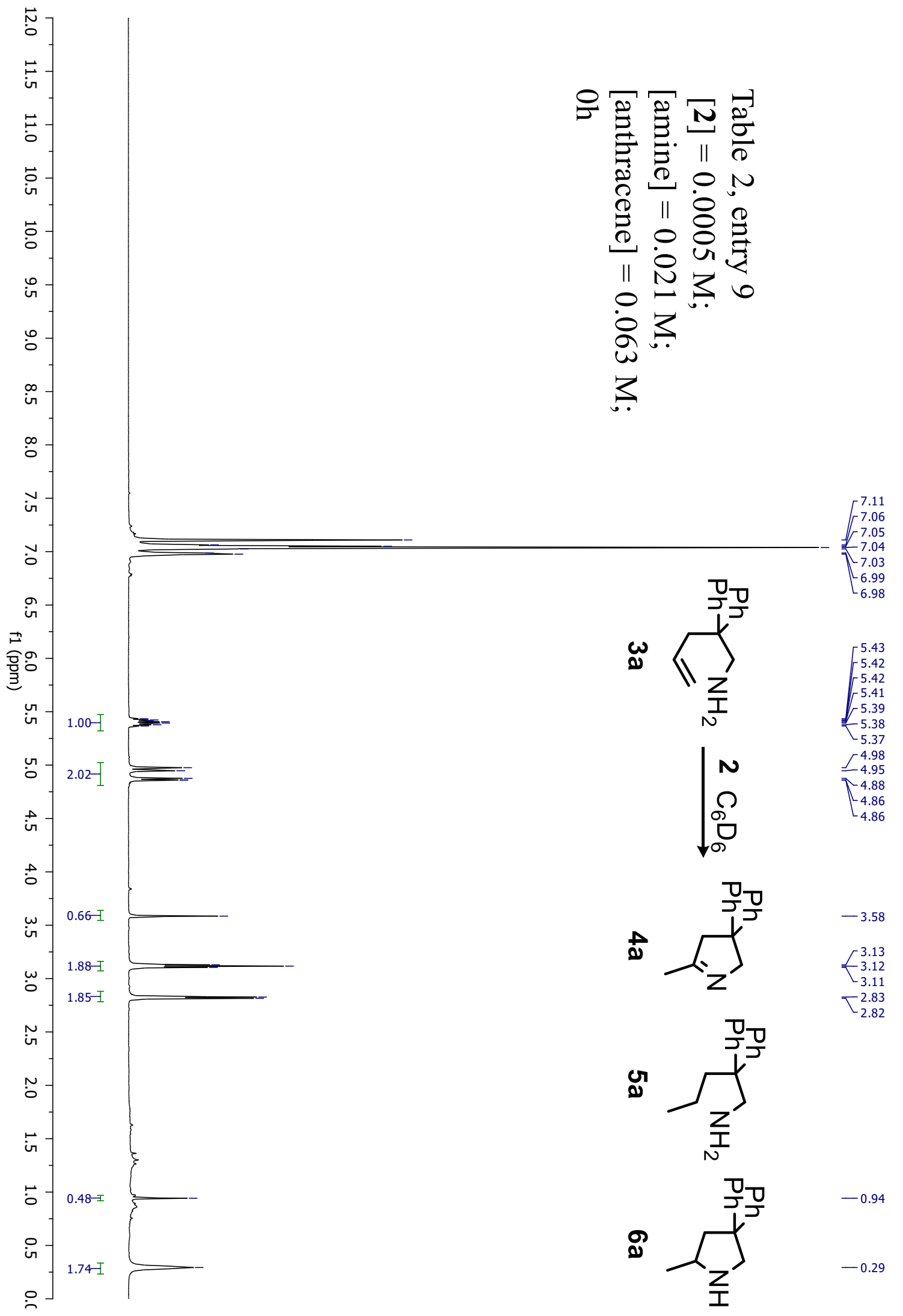

Figure S26. ${ }^{1} \mathrm{H}$ NMR spectrum for Table 2, entry 9. 


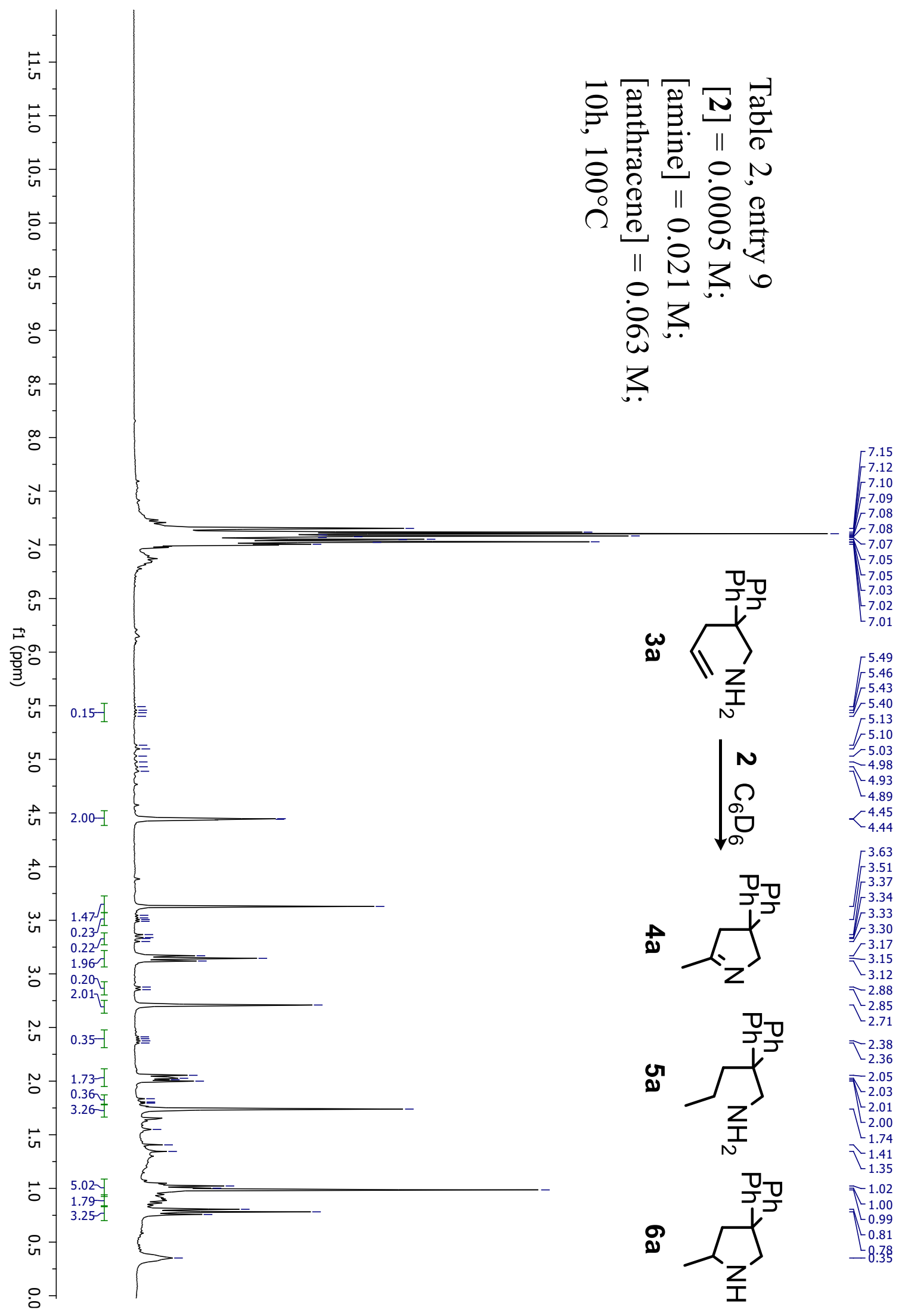

Figure S27. ${ }^{1} \mathrm{H}$ NMR spectrum for Table 2, entry 9. 


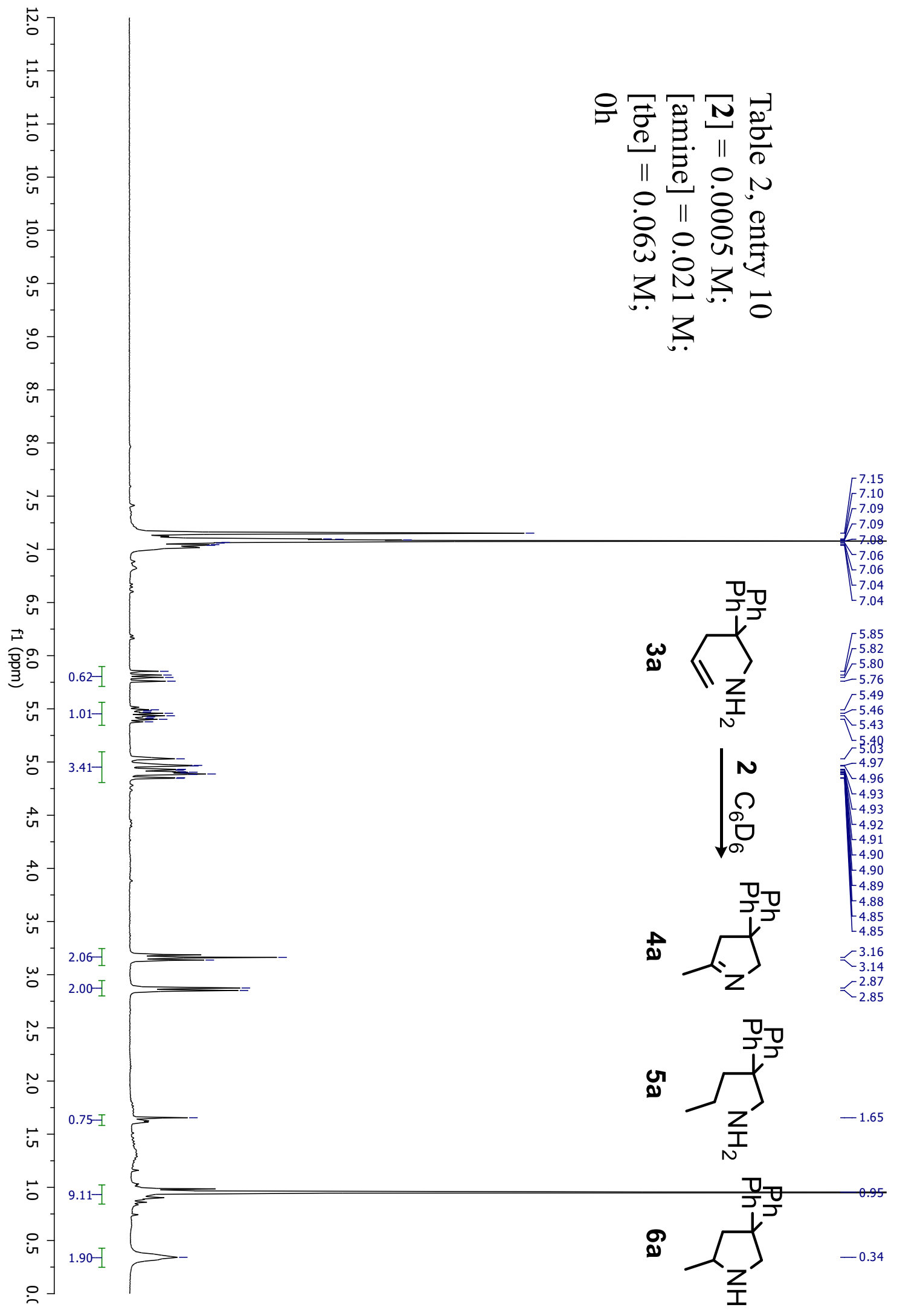

Figure S28. ${ }^{1} \mathrm{H}$ NMR spectrum for Table 2, entry 10. 


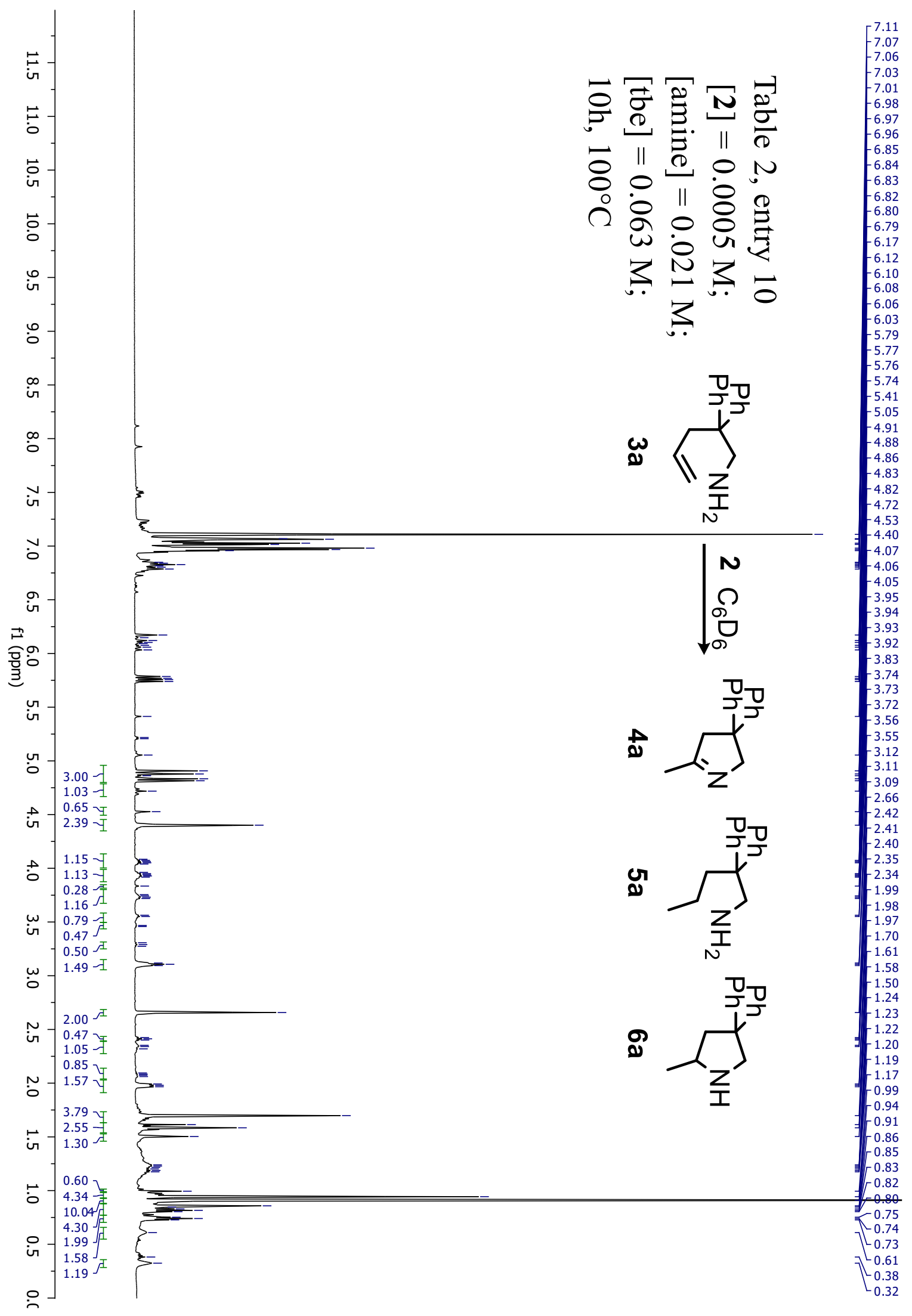

Figure S29. ${ }^{1} \mathrm{H}$ NMR spectrum for Table 2, entry 10. 


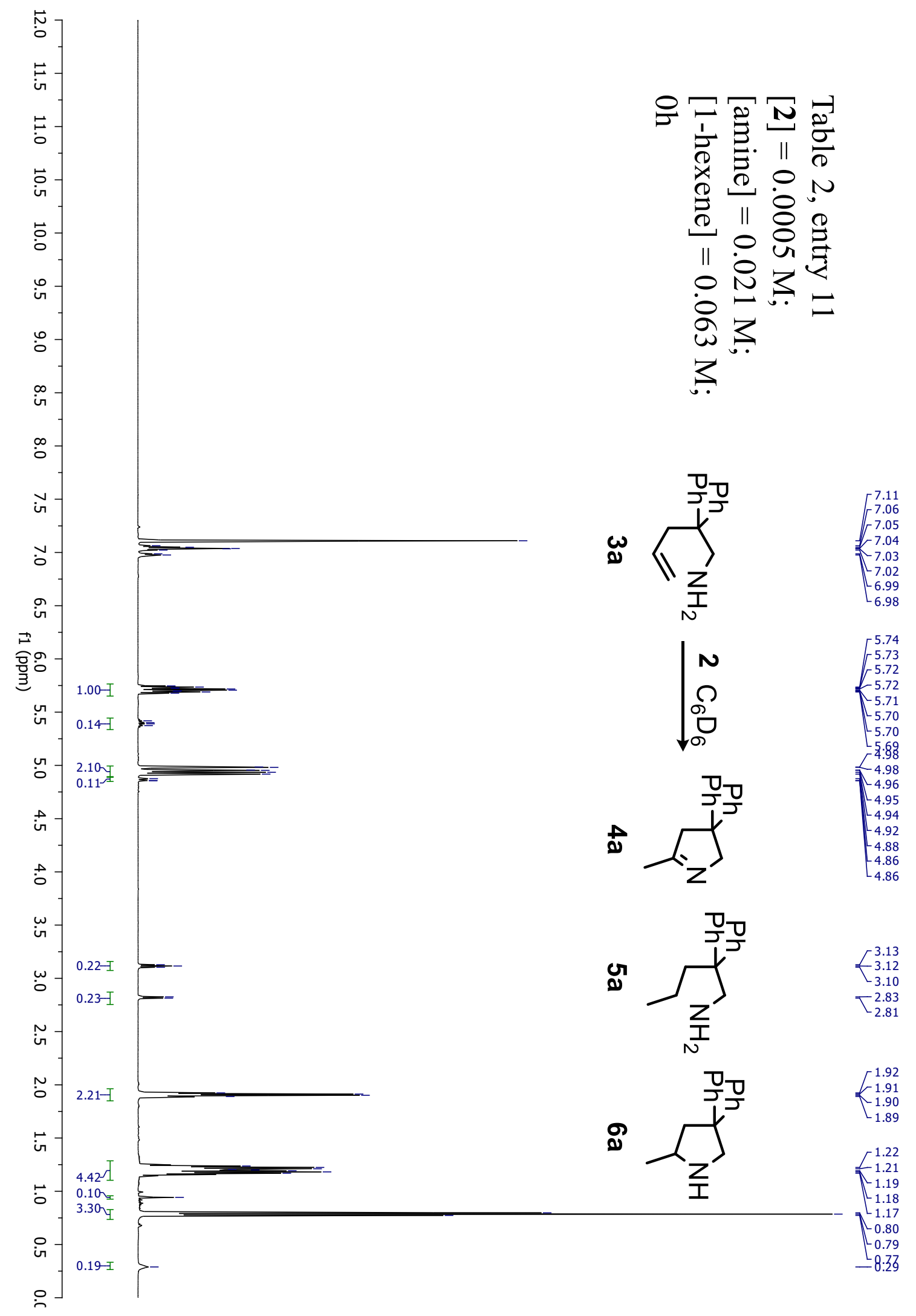

Figure S30. ${ }^{1} \mathrm{H}$ NMR spectrum for Table 2, entry 11. 


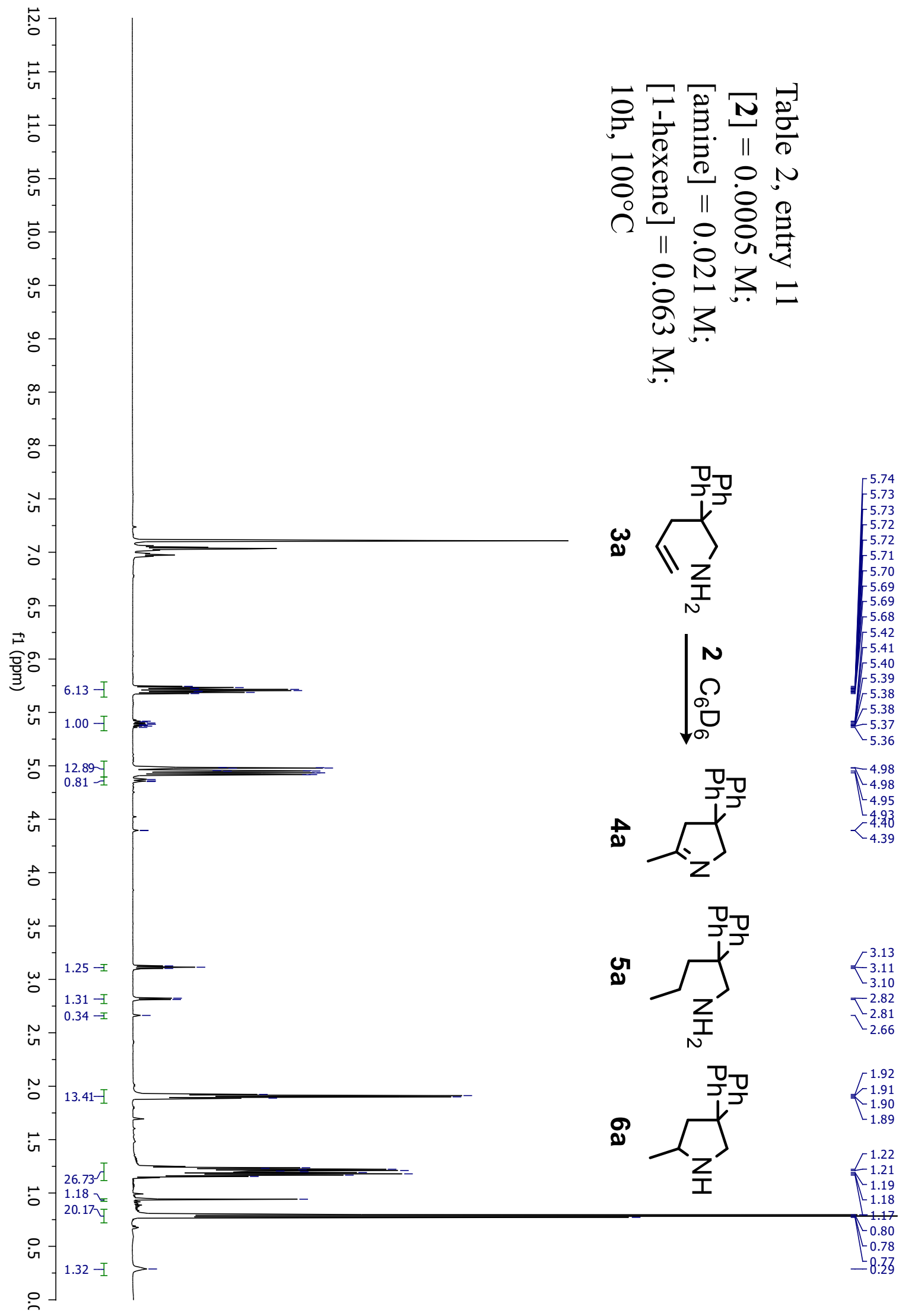

Figure S31. ${ }^{1} \mathrm{H}$ NMR spectrum for Table 2, entry 11. 


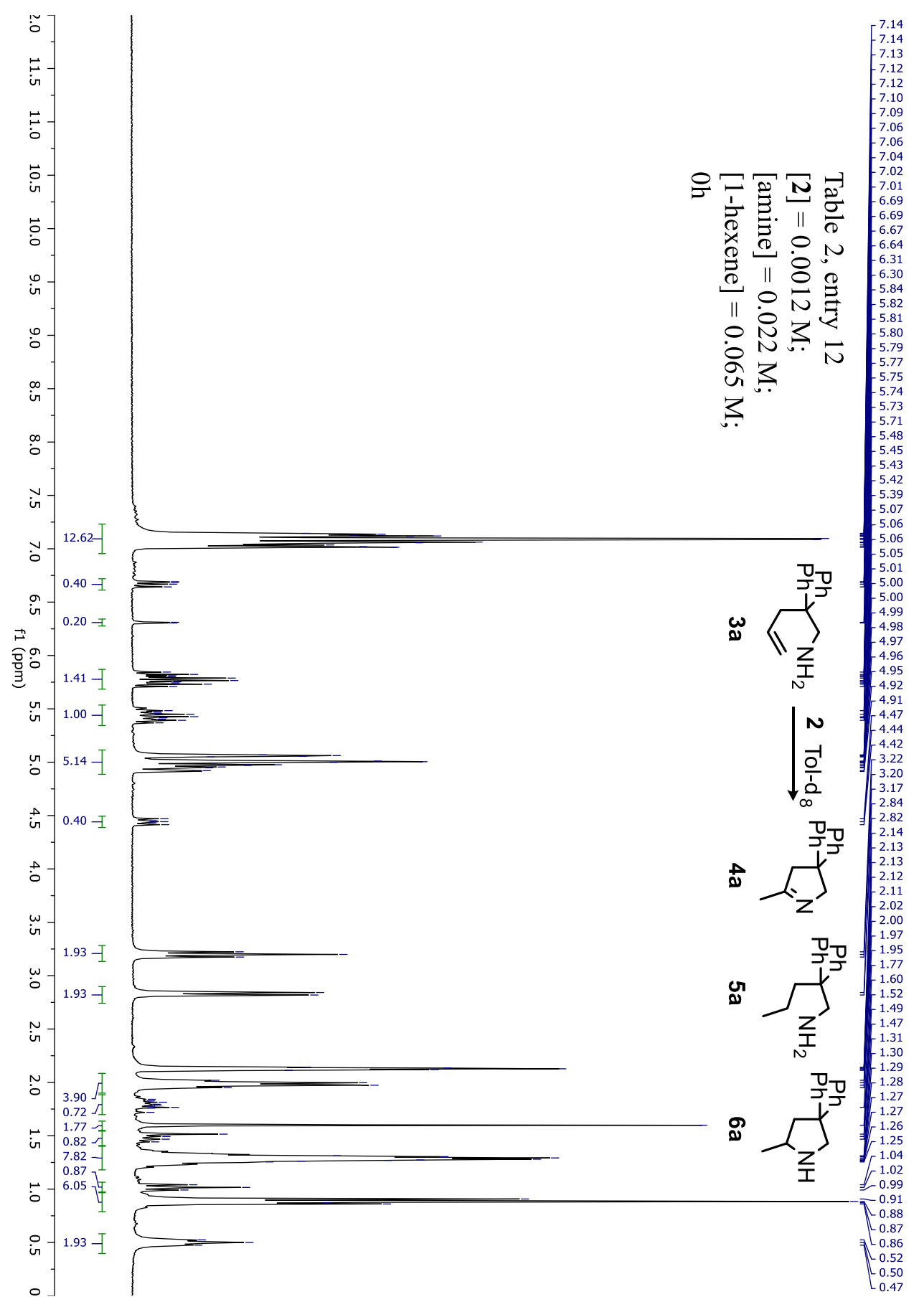

Figure S32. ${ }^{1} \mathrm{H}$ NMR spectrum for Table 2, entry 12. 


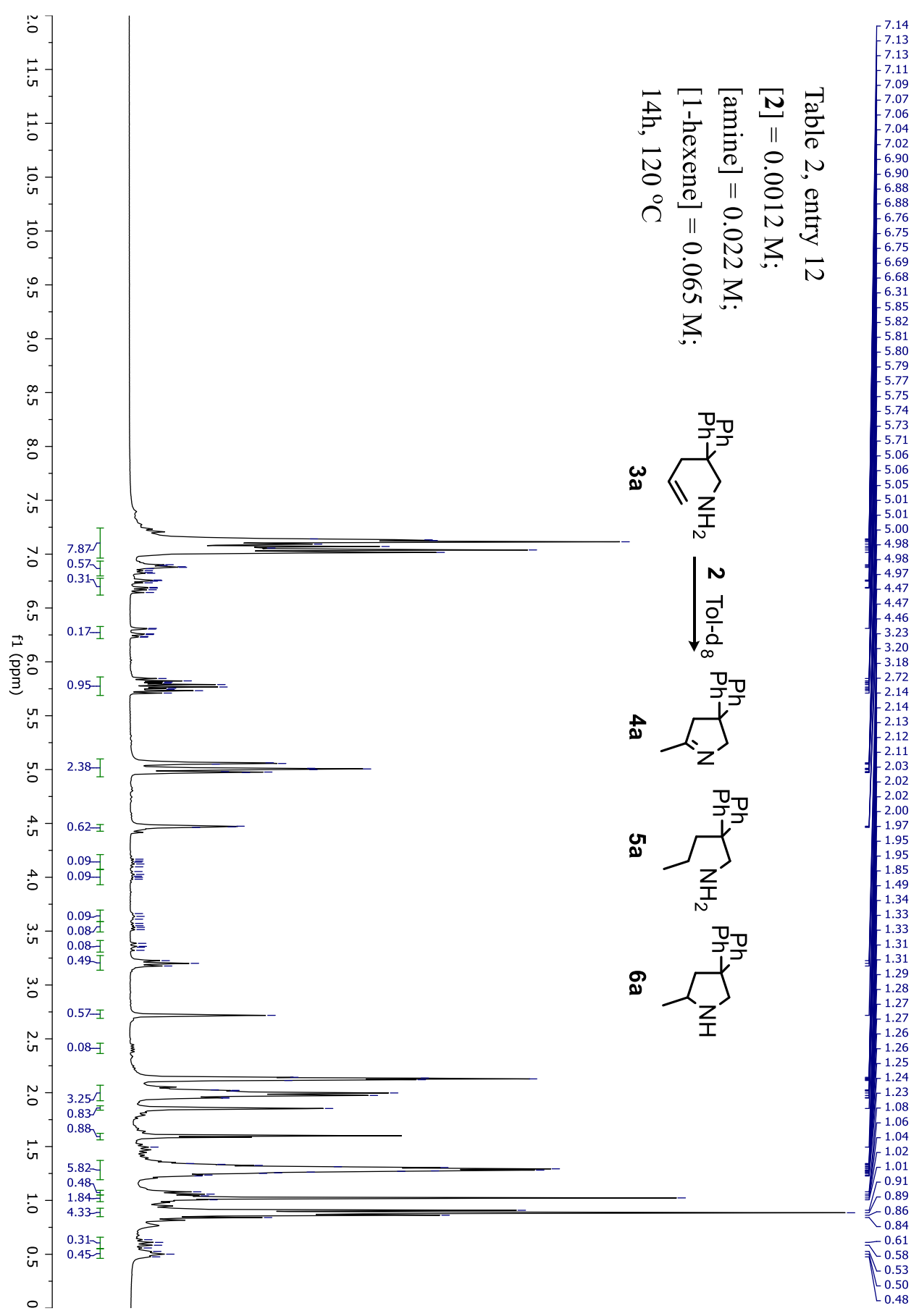

Figure S33. ${ }^{1} \mathrm{H}$ NMR spectrum for Table 2, entry 12. 
Spectrum of 2:
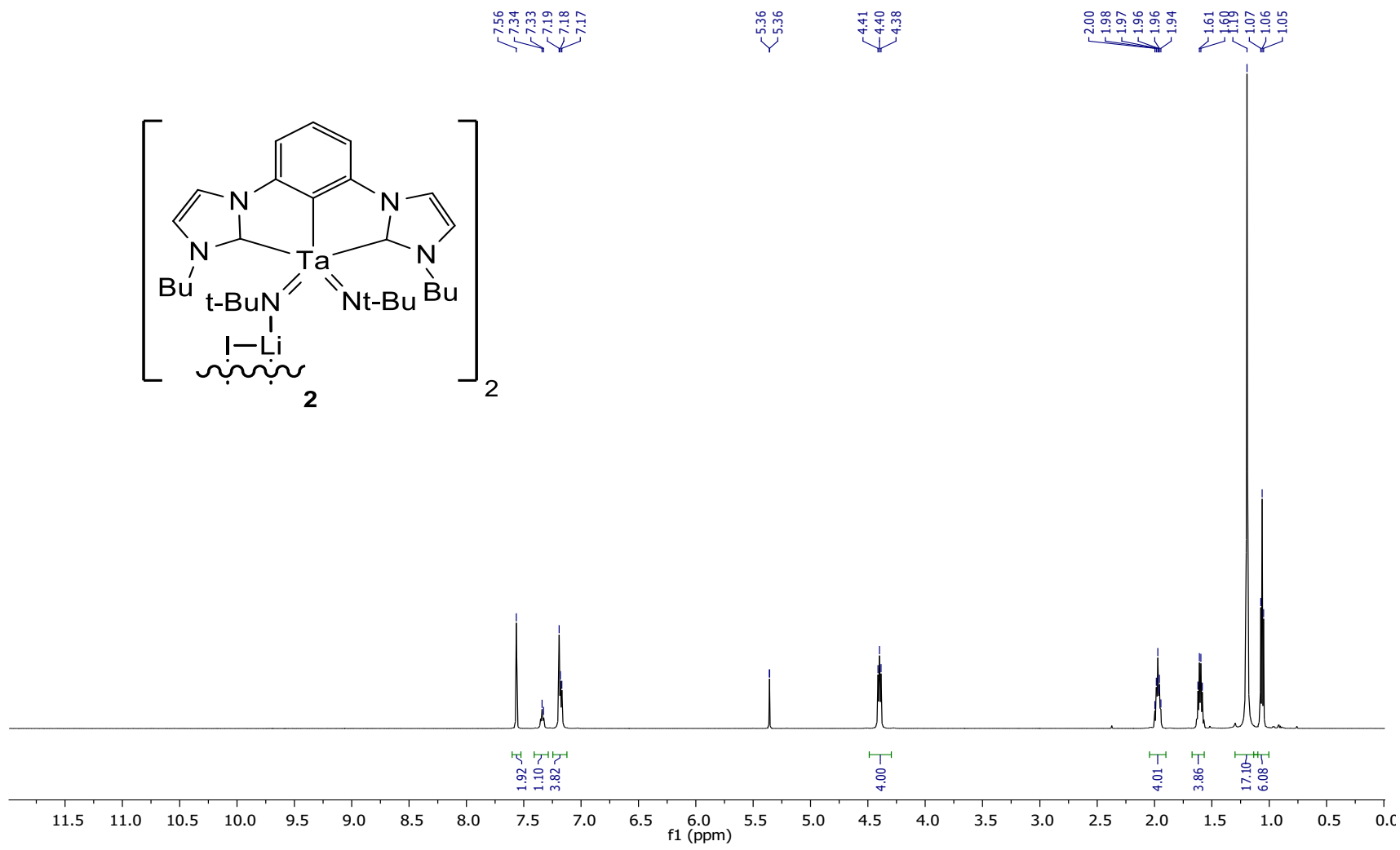

Figure S34. ${ }^{1} \mathrm{H}$ NMR spectrum for complex 2.
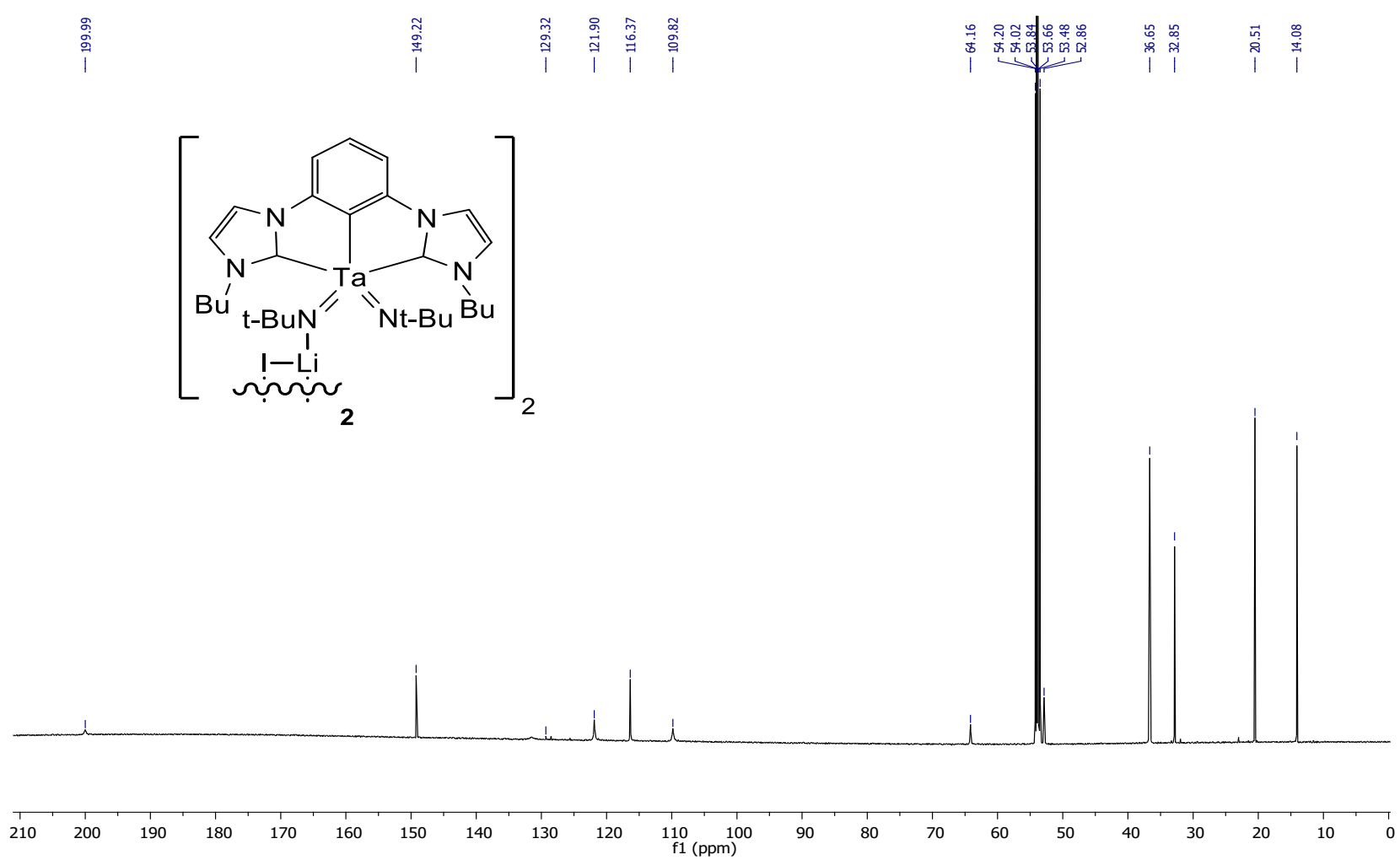

Figure S35. ${ }^{13} \mathrm{C}$ NMR spectrum for complex 2. 


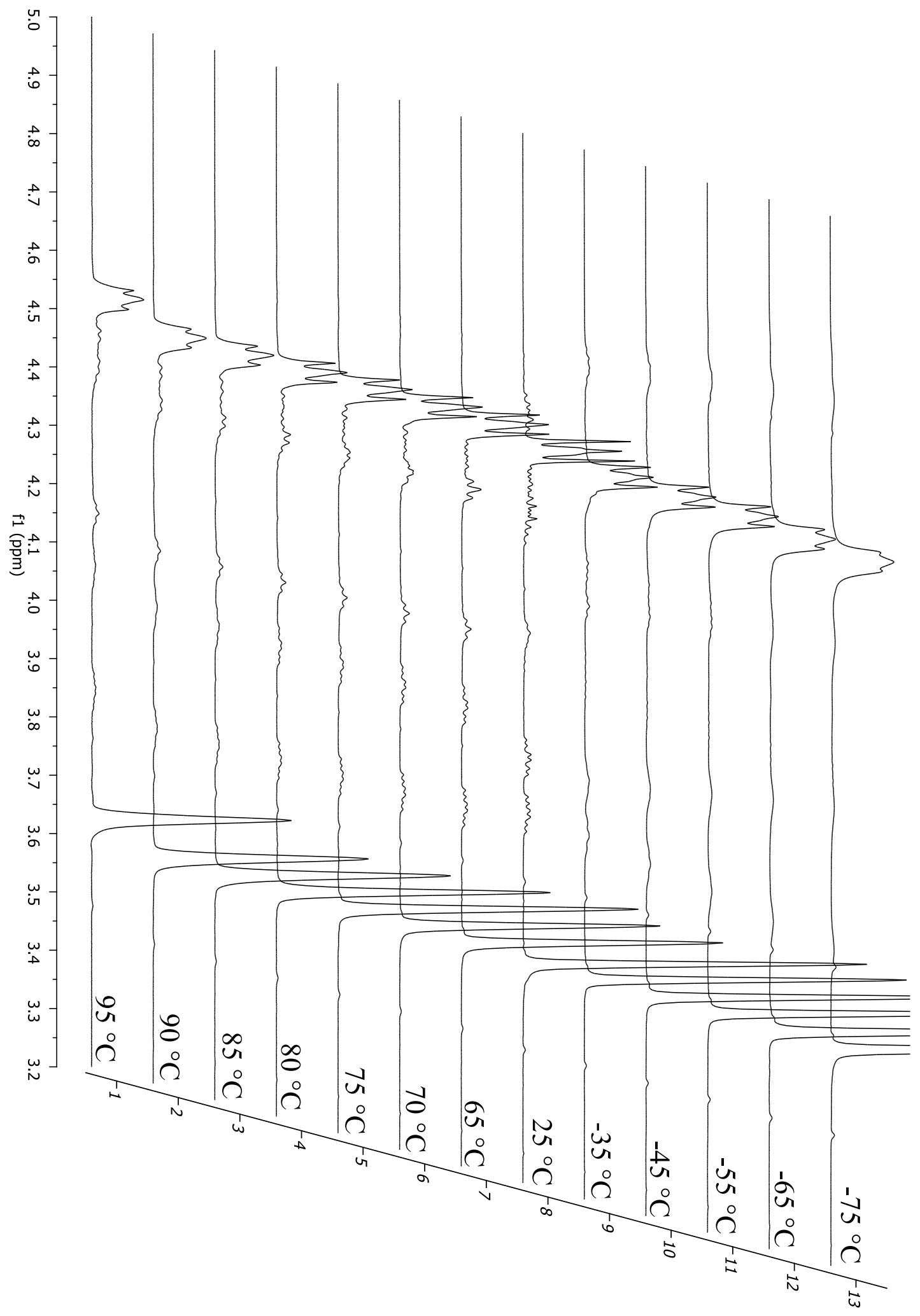

Figure S36. VT-NMR spectra of 2 were collected on a Bruker Avance $500 \mathrm{MHz}$ NMR instrument. THF-d8 was used as solvent. 
HRMS:

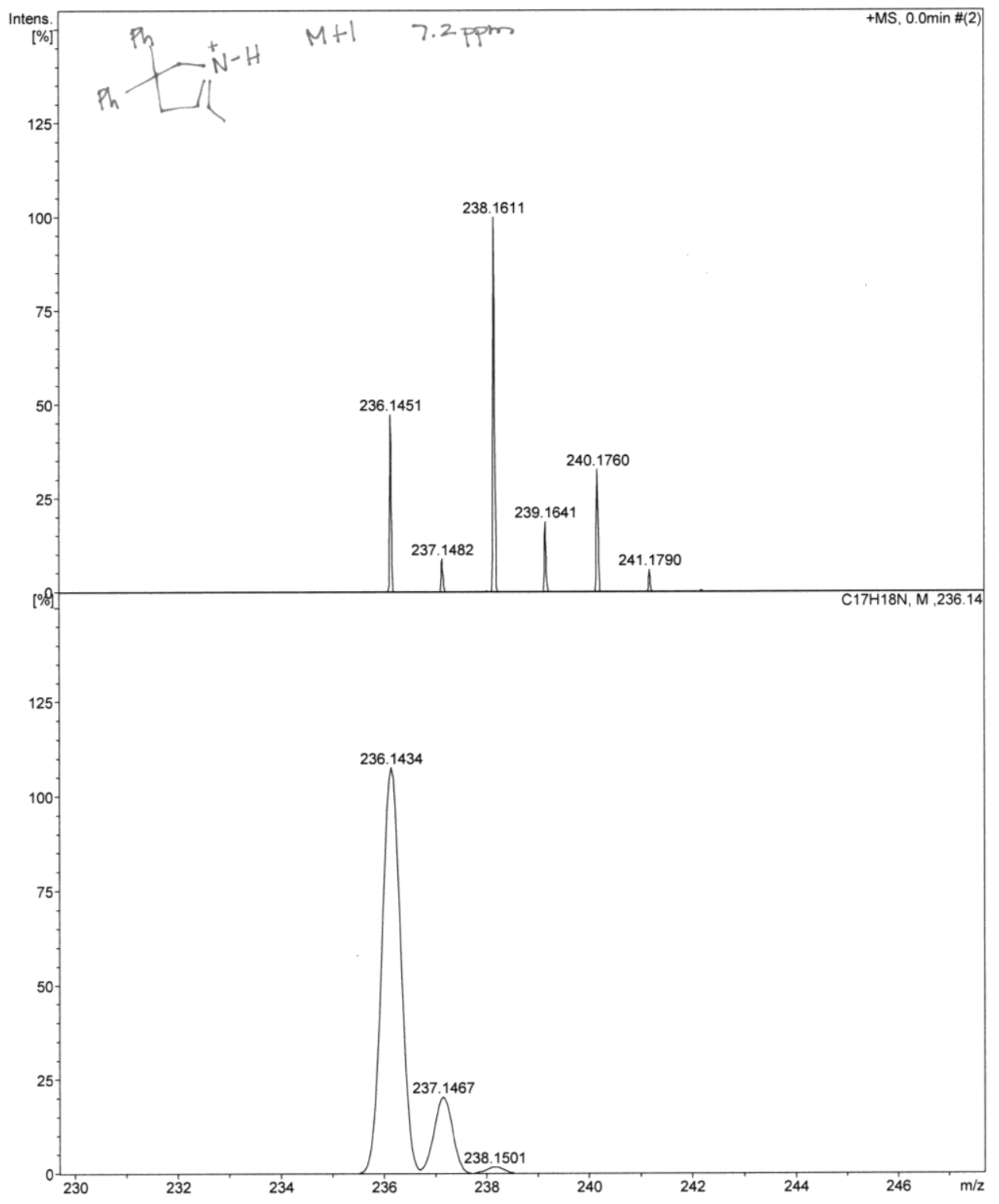

Figure S37. HRMS spectrum of catalytic trial (Entry 1, OA product)

HRMS (ESI) $m / z$ calculated for $\mathrm{C}_{17} \mathrm{H}_{18} \mathrm{~N}[\mathrm{M}+\mathrm{H}]^{+} 236.1434$ (bottom), found 236.1451 (top) 


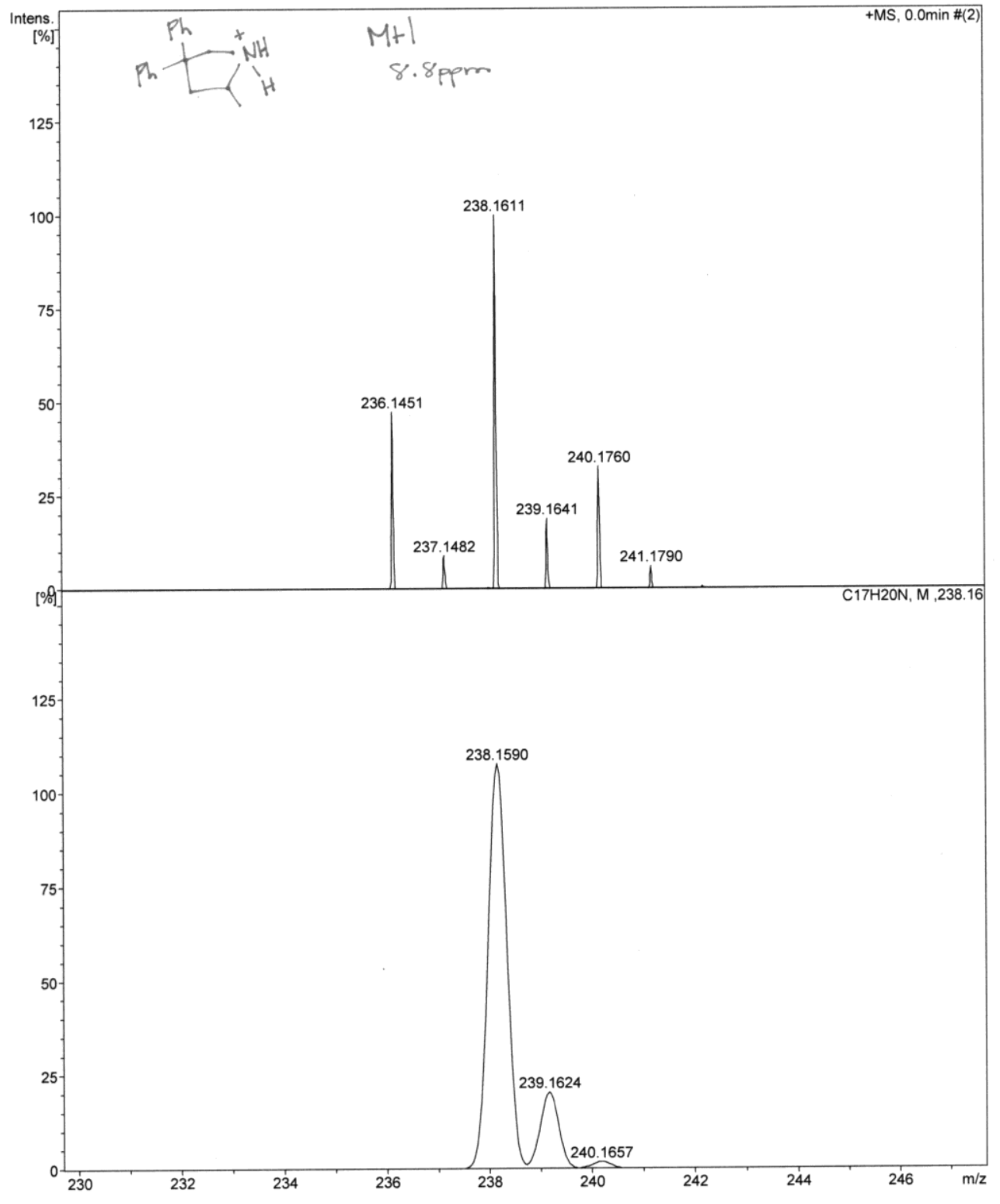

Figure S38. HRMS spectrum of catalytic trial (Entry 1, HA product)

HRMS (ESI) $m / z$ calculated for $\mathrm{C}_{17} \mathrm{H}_{20} \mathrm{~N}[\mathrm{M}+\mathrm{H}]^{+} 238.1590$ (bottom), found 238.1611 (top) 


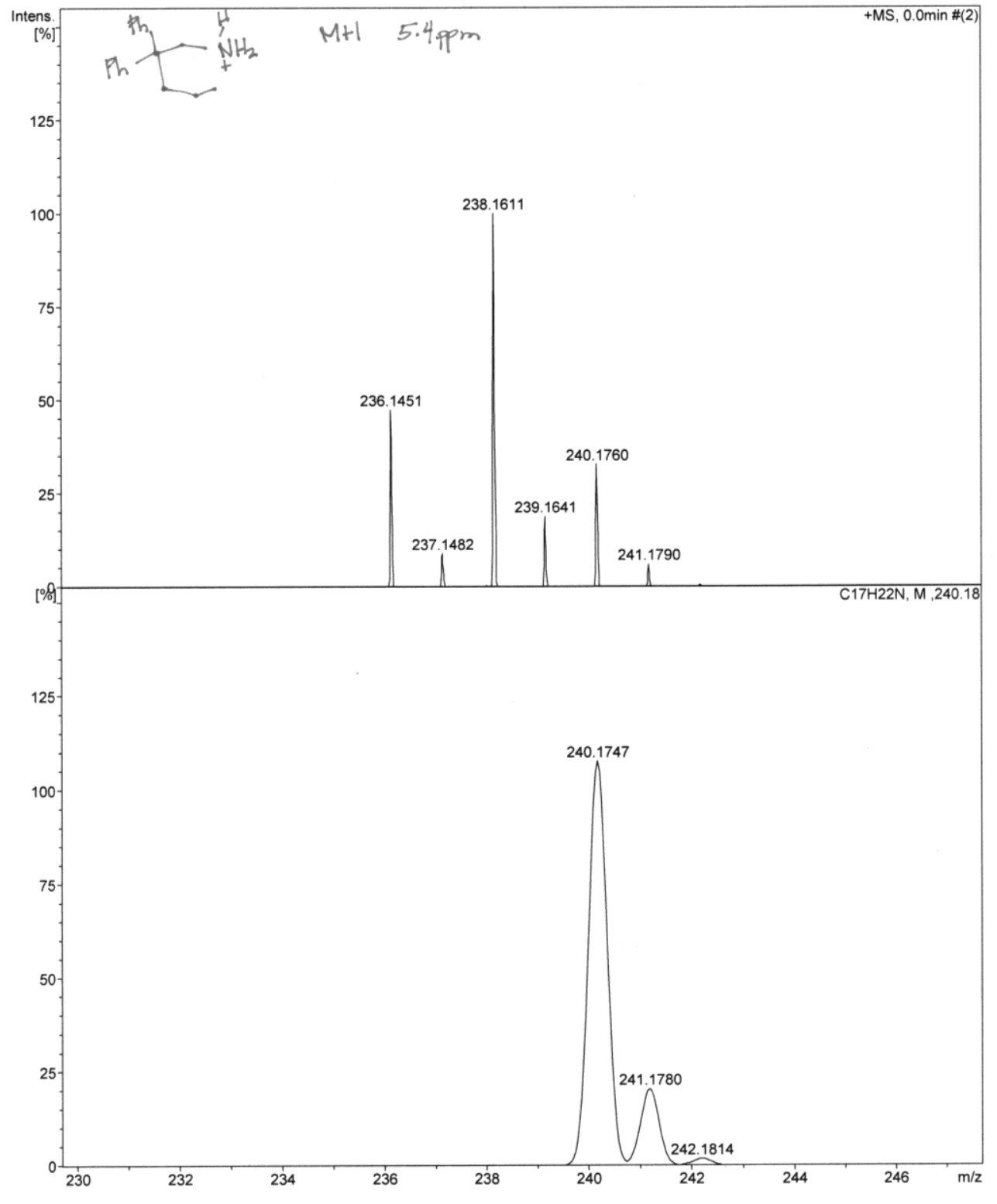

Figure S39. HRMS spectrum of catalytic trial (Entry 1, saturated product)

HRMS (ESI) $m / z$ calculated for $\mathrm{C}_{17} \mathrm{H}_{22} \mathrm{~N}[\mathrm{M}+\mathrm{H}]^{+} 240.1747$ (bottom), found 240.1760 (top) 


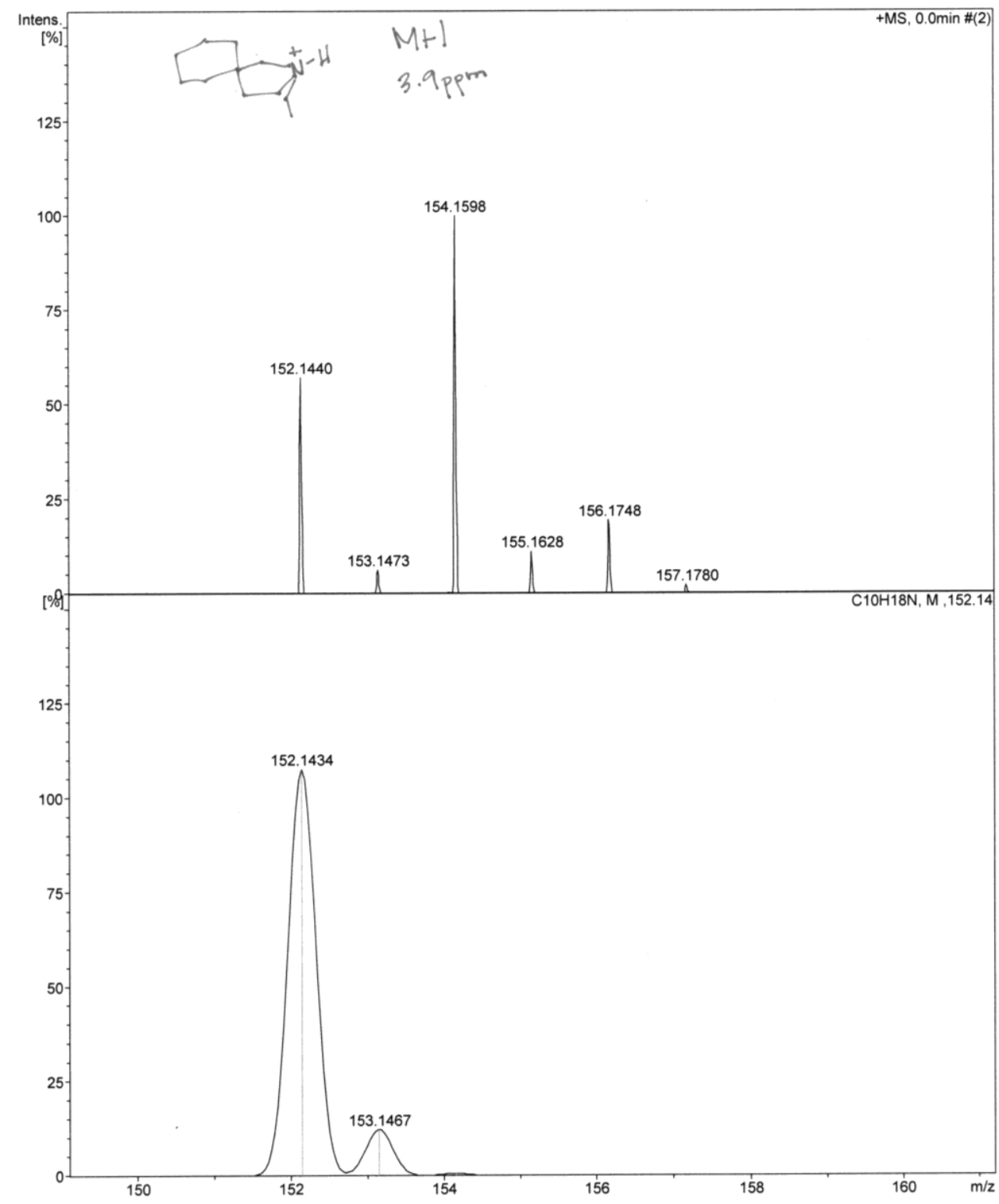

Figure S40. HRMS spectrum of catalytic trial (Entry 3, OA product)

HRMS (ESI) $m / z$ calculated for $\mathrm{C}_{10} \mathrm{H}_{18} \mathrm{~N}[\mathrm{M}+\mathrm{H}]^{+} 152.1434$ (bottom), found 152.1440 (top) 


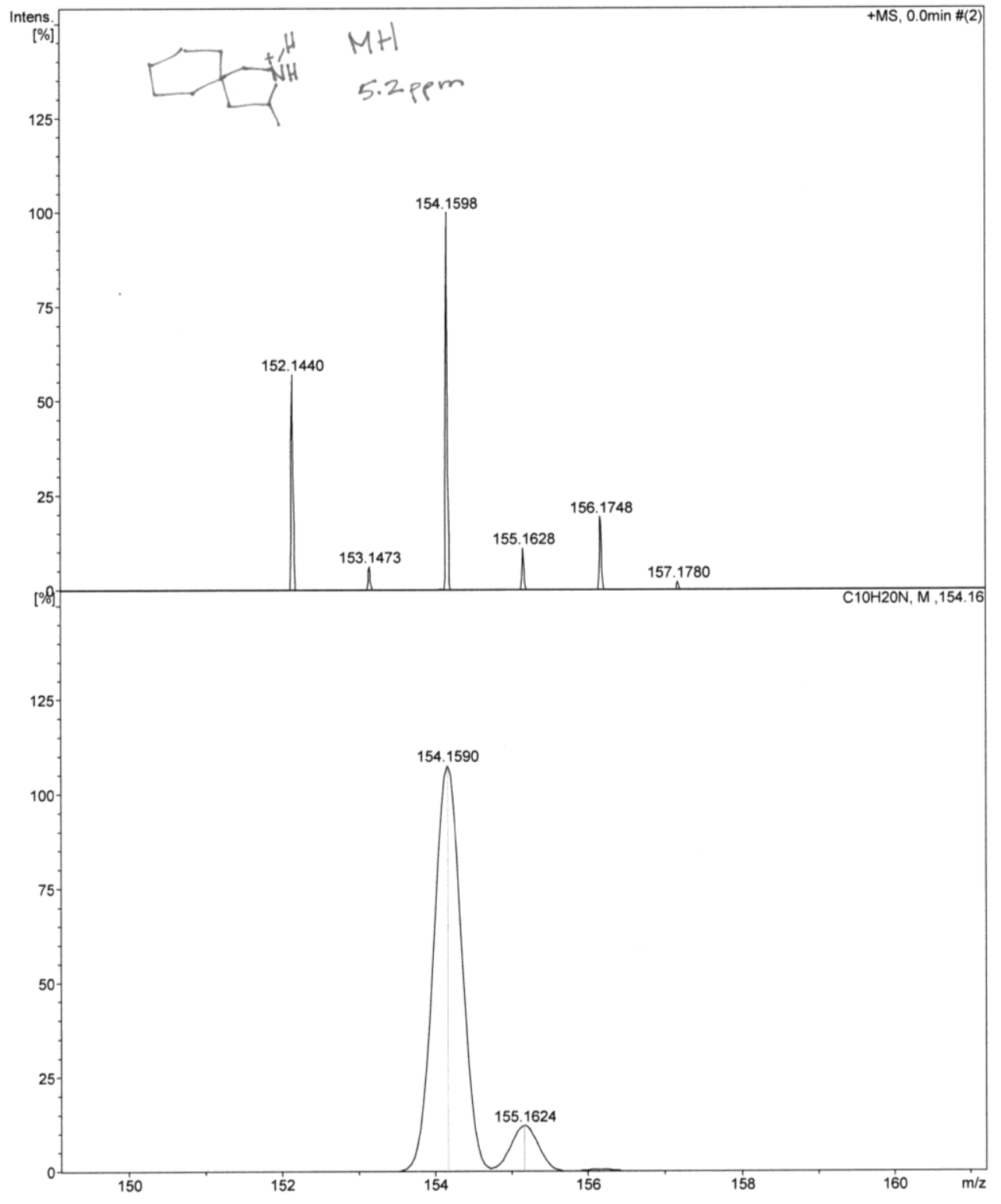

Figure S41. HRMS spectrum of catalytic trial (Entry 3, HA product)

HRMS (ESI) $m / z$ calculated for $\mathrm{C}_{10} \mathrm{H}_{20} \mathrm{~N}[\mathrm{M}+\mathrm{H}]^{+} 154.1590$ (bottom), found 154.1598 (top) 


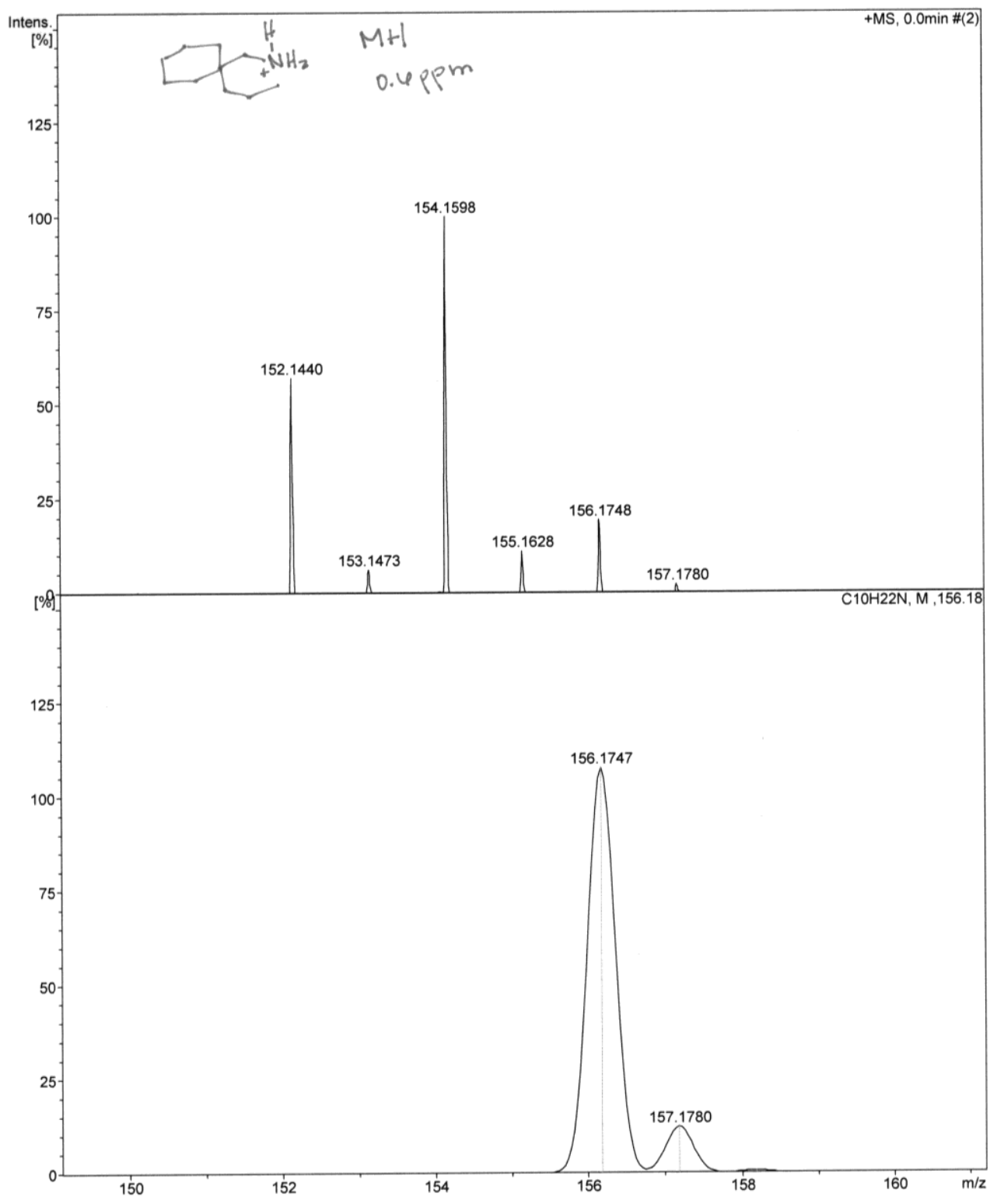

Figure S42. HRMS spectrum of catalytic trial (Entry 3, saturated product) HRMS (ESI) $m / z$ calculated for $\mathrm{C}_{10} \mathrm{H}_{22} \mathrm{~N}[\mathrm{M}+\mathrm{H}]^{+} 156.1747$ (bottom), found 156.1748 (top) 


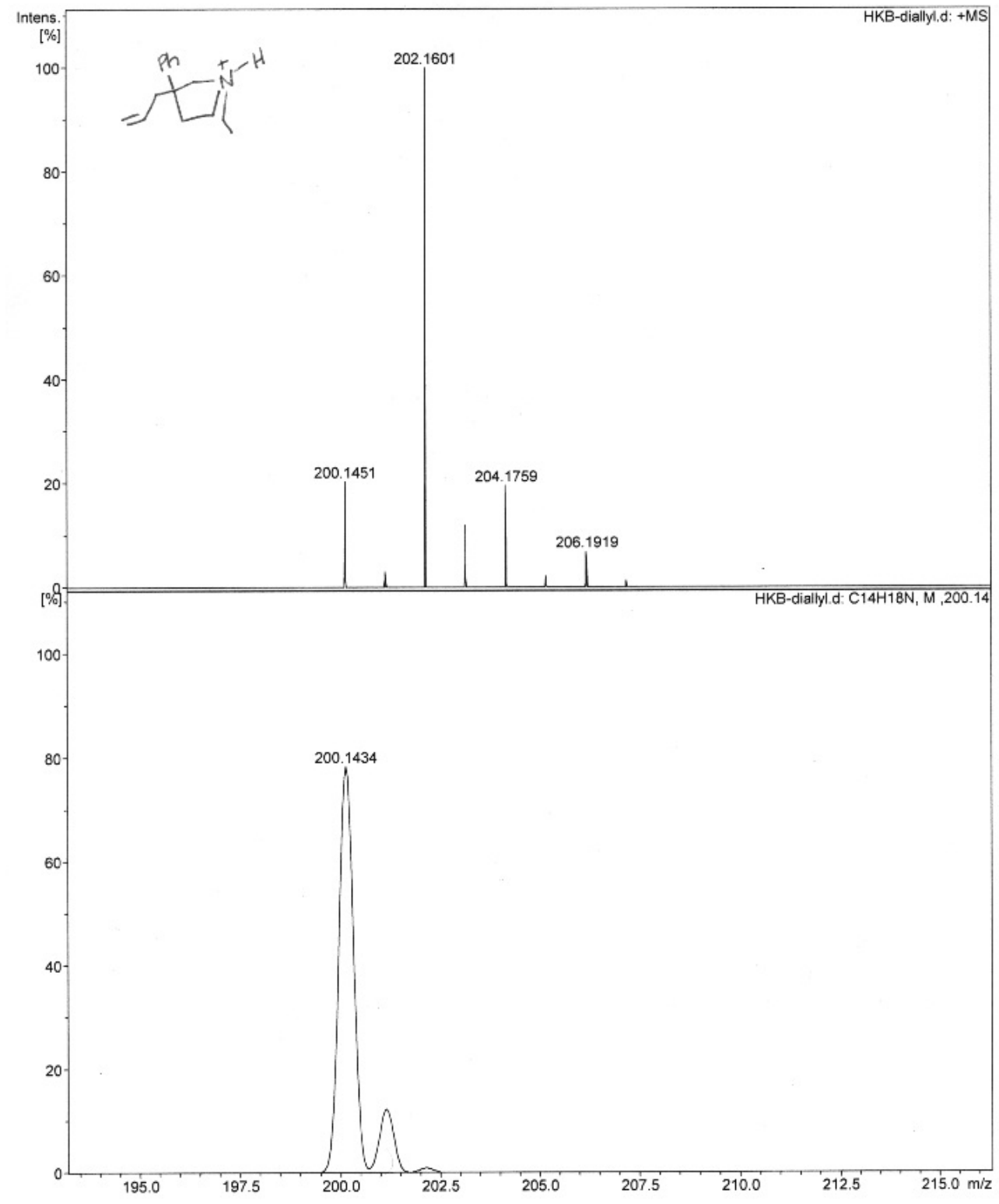

Figure S43. HRMS spectrum of catalytic trial (Entry 5, OA product)

HRMS (ESI) $m / z$ calculated for $\mathrm{C}_{14} \mathrm{H}_{18} \mathrm{~N}[\mathrm{M}+\mathrm{H}]^{+} 200.1434$ (bottom), found 200.1451 (top) 


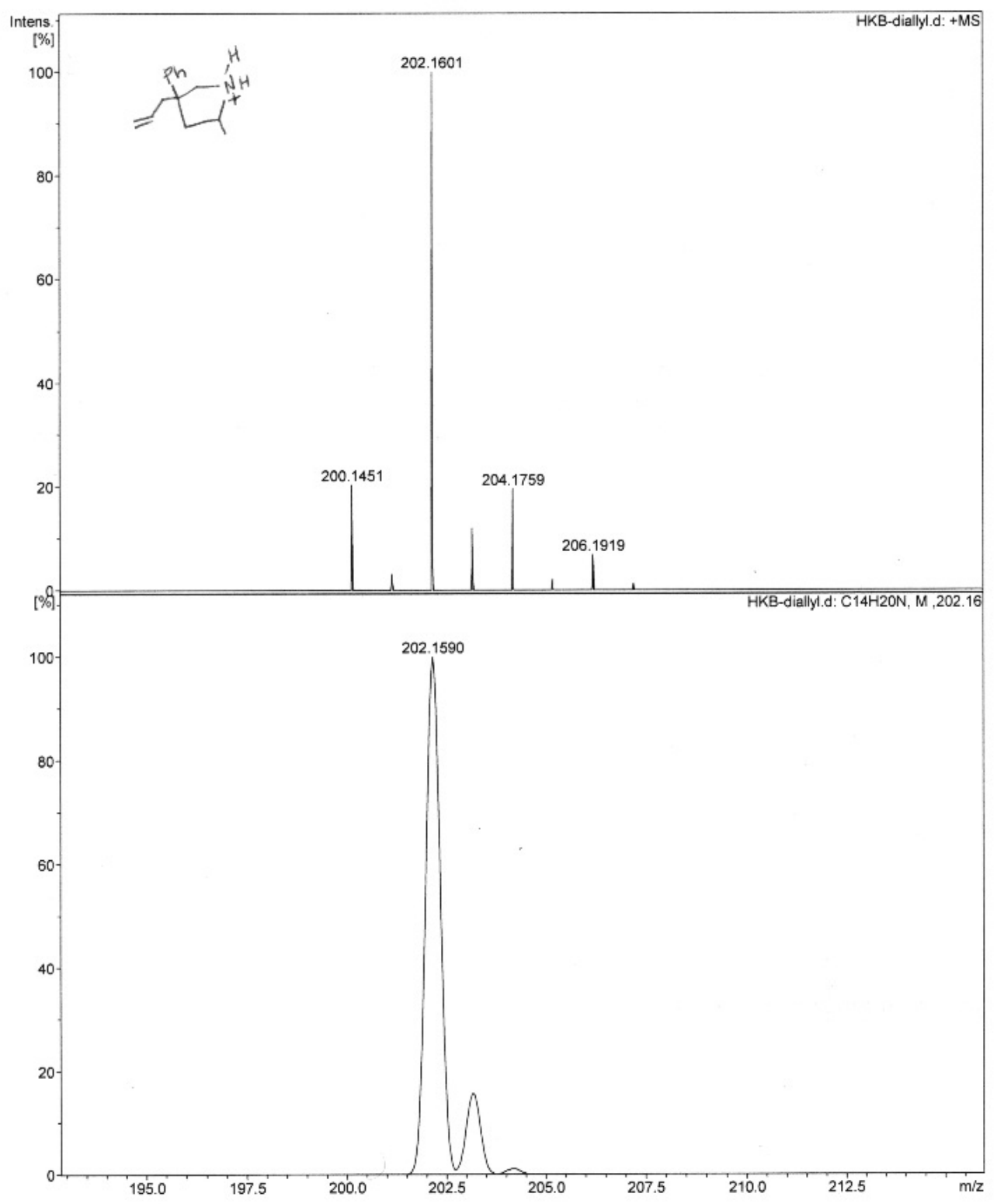

Figure S44. HRMS spectrum of catalytic trial (Entry 5, HA product) HRMS (ESI) $m / z$ calculated for $\mathrm{C}_{14} \mathrm{H}_{20} \mathrm{~N}[\mathrm{M}+\mathrm{H}]^{+} 202.1590$ (bottom), found 202.1601 (top) 


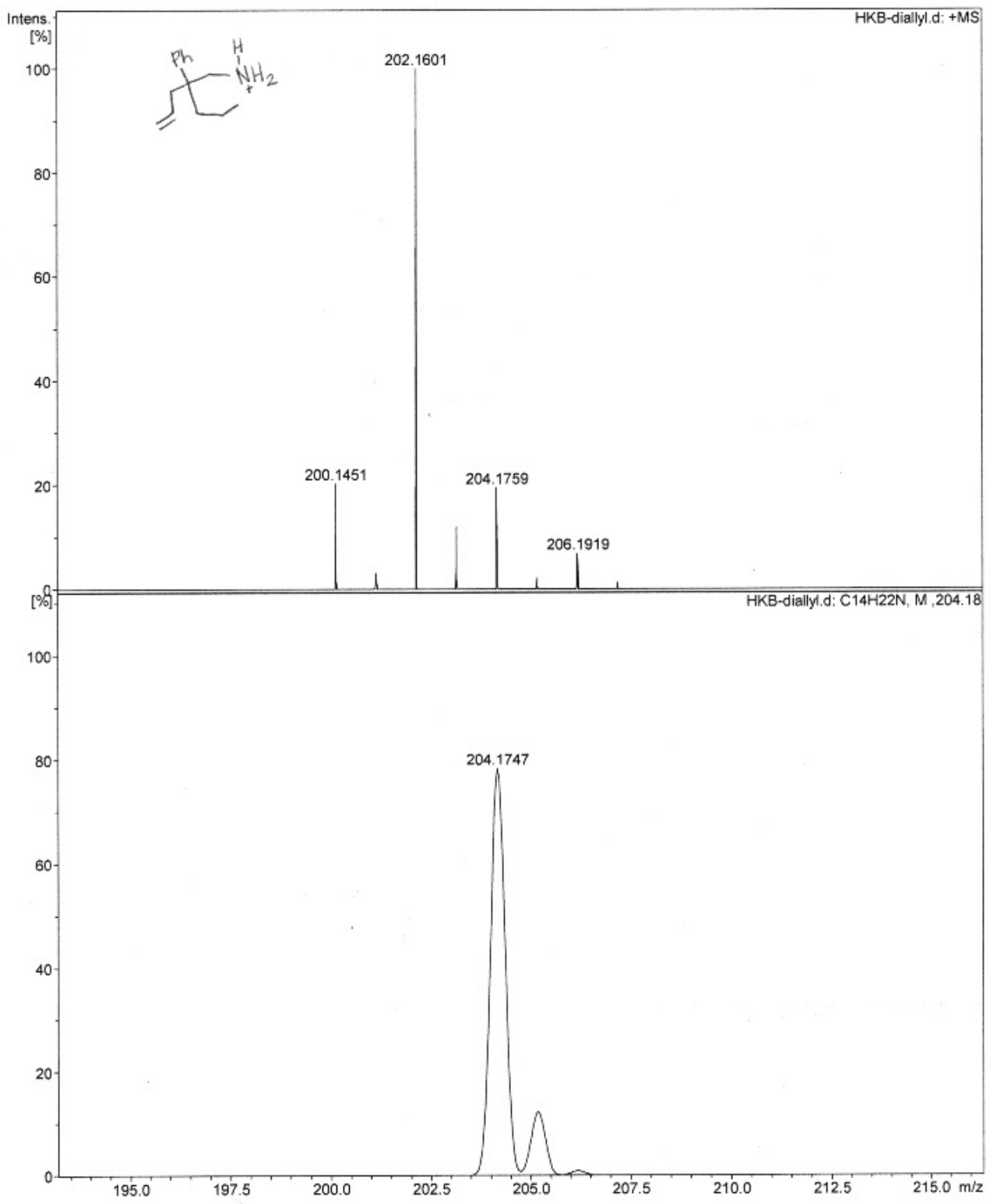

Figure S45. HRMS spectrum of catalytic trial (Entry 5, saturated product) HRMS (ESI) $m / z$ calculated for $\mathrm{C}_{14} \mathrm{H}_{22} \mathrm{~N}[\mathrm{M}+\mathrm{H}]^{+} 204.1747$ (bottom), found 204.1759 (top) 


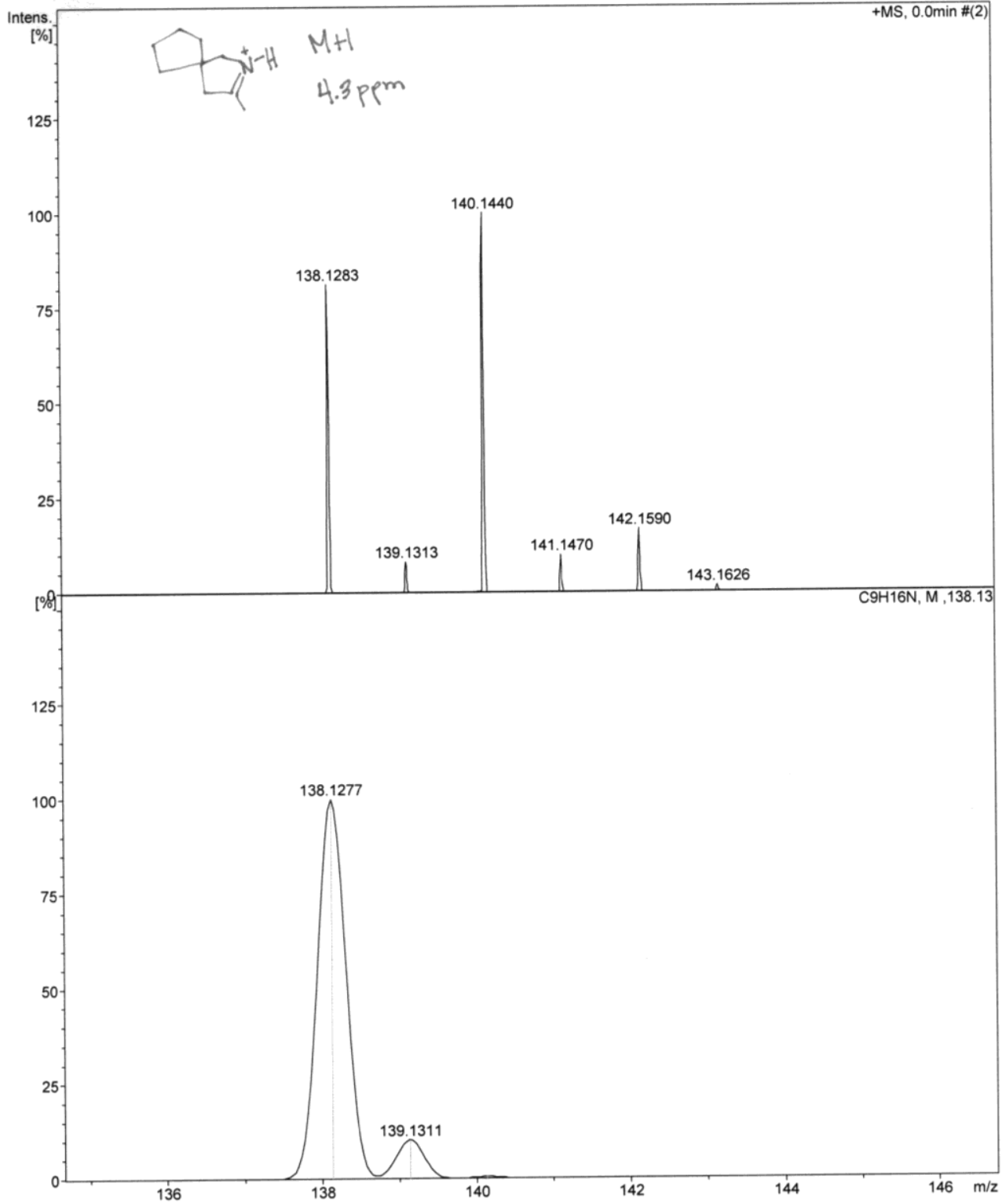

Figure S46. HRMS spectrum of catalytic trial (Entry 7, OA product)

HRMS (ESI) $m / z$ calculated for $\mathrm{C}_{9} \mathrm{H}_{16} \mathrm{~N}[\mathrm{M}+\mathrm{H}]^{+} 138.1277$ (bottom), found 138.1283 (top) 


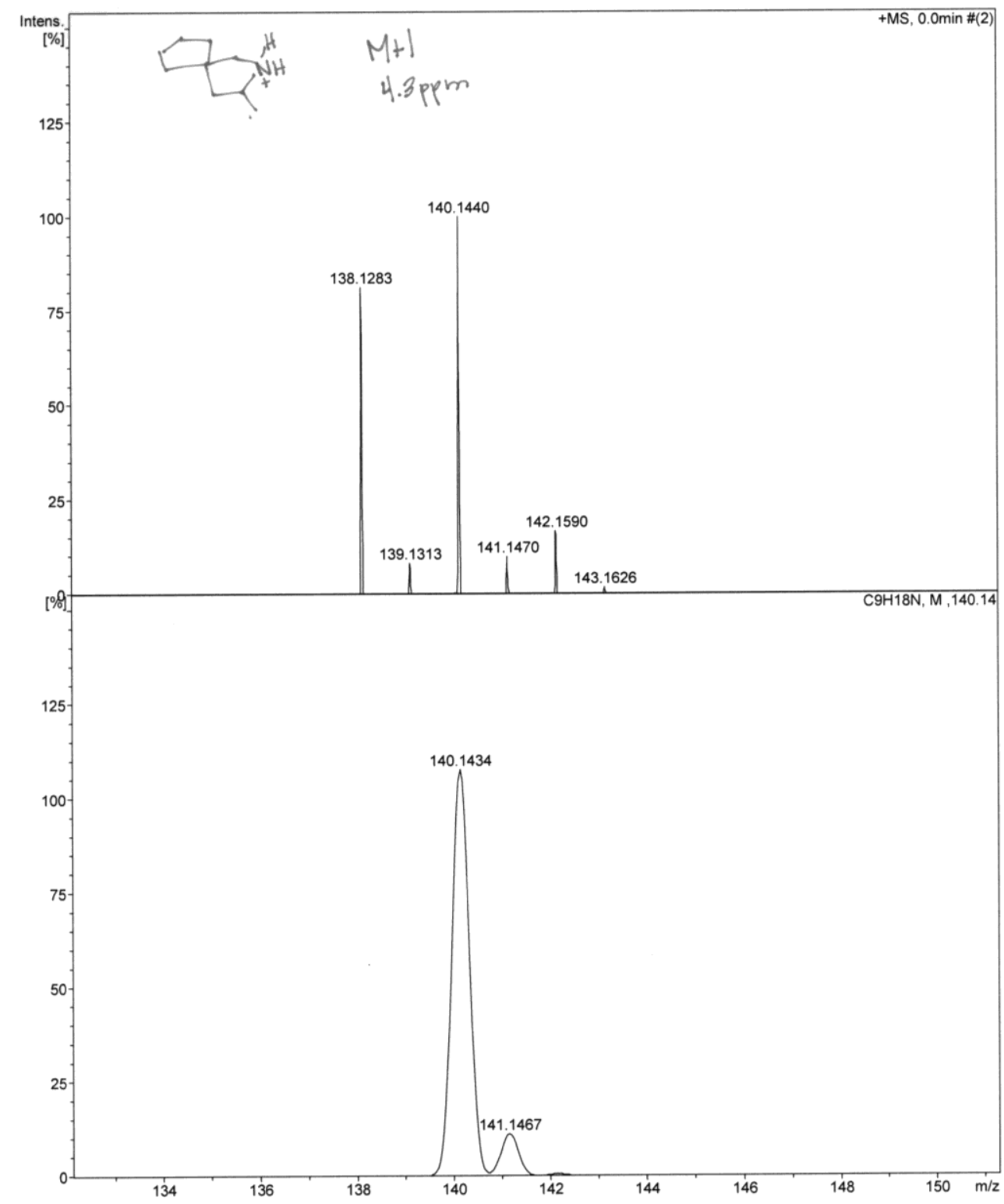

Figure S47. HRMS spectrum of catalytic trial (Entry 7, HA product) HRMS (ESI) $m / z$ calculated for $\mathrm{C}_{9} \mathrm{H}_{18} \mathrm{~N}[\mathrm{M}+\mathrm{H}]^{+} 140.1434$ (bottom), found 140.1440 (top) 


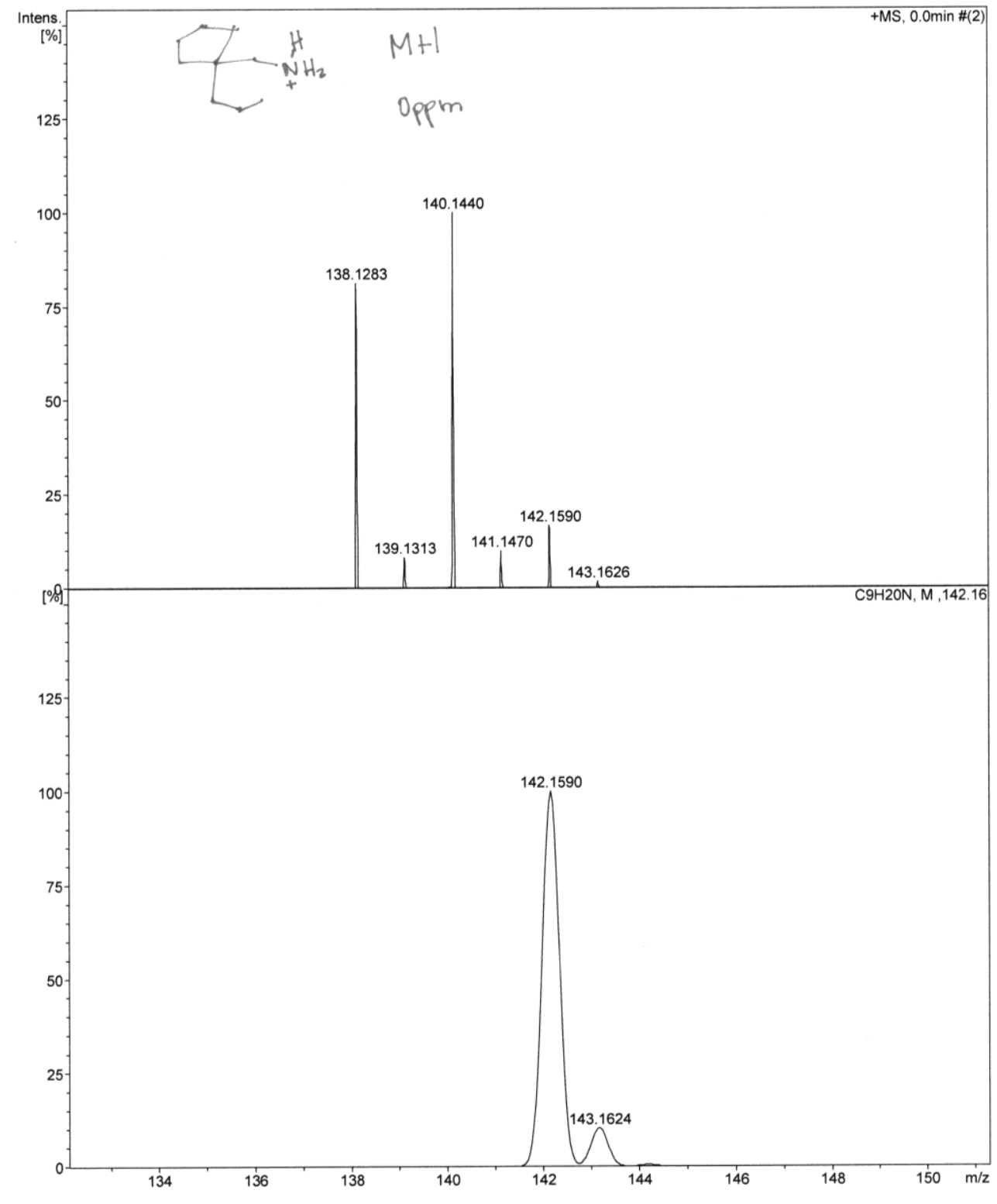

Figure S48. HRMS spectrum of catalytic trial (Entry 7, saturated product)

HRMS (ESI) $m / z$ calculated for $\mathrm{C}_{9} \mathrm{H}_{20} \mathrm{~N}[\mathrm{M}+\mathrm{H}]^{+} 142.1590$ (bottom), found 142.1590 (top) 


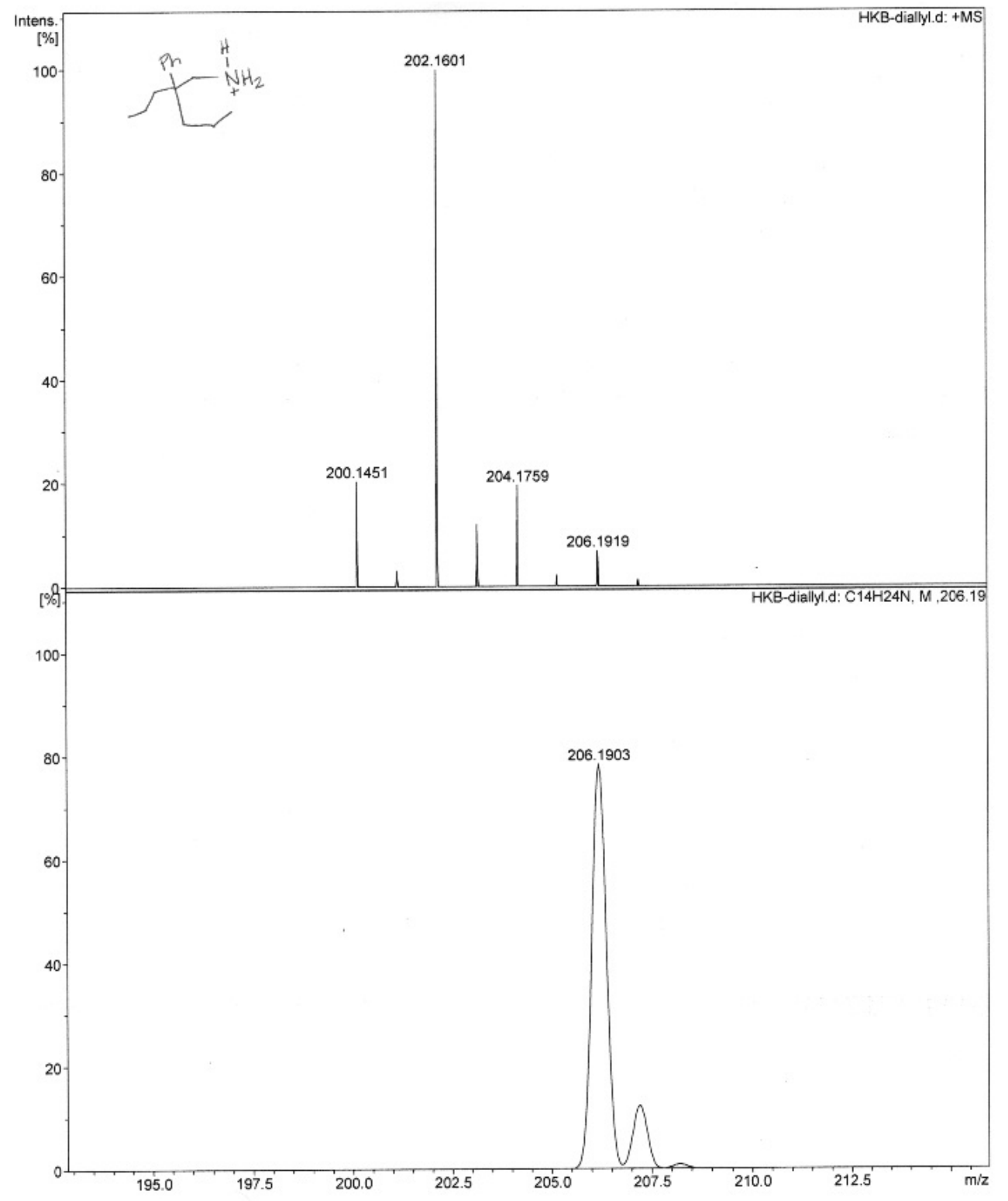

Figure S49. HRMS spectrum of catalytic trial (Entry 8, internal hydrogen acceptor) HRMS (ESI) $m / z$ calculated for $\mathrm{C}_{14} \mathrm{H}_{24} \mathrm{~N}[\mathrm{M}+\mathrm{H}]^{+} 206.1903$ (bottom), found 206.1919 (top) 


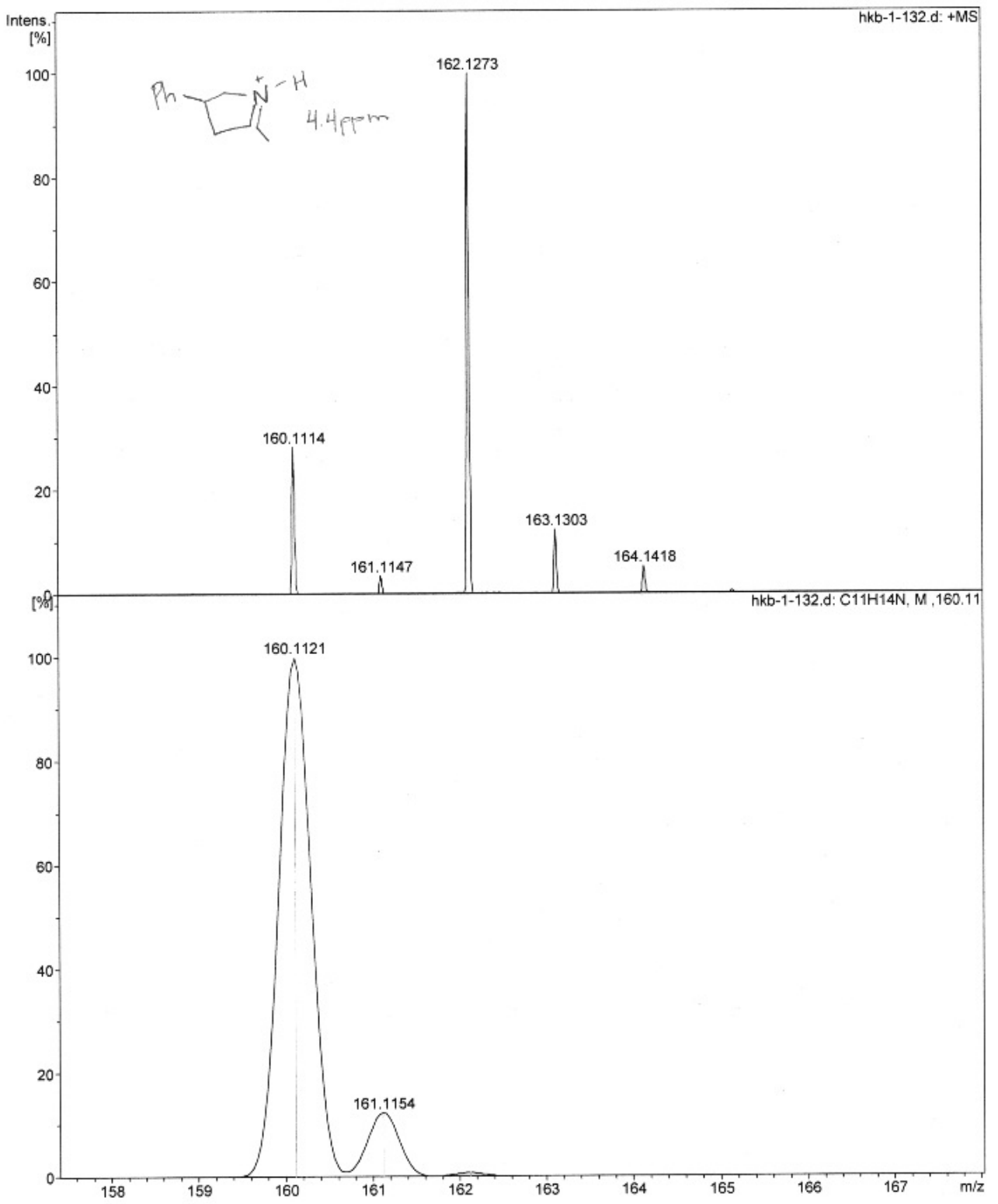

Figure S50. HRMS spectrum of catalytic trial (Entry 8, OA product)

HRMS (ESI) $m / z$ calculated for $\mathrm{C}_{11} \mathrm{H}_{14} \mathrm{~N}[\mathrm{M}+\mathrm{H}]^{+} 160.1121$ (bottom), found 160.1114 (top) 


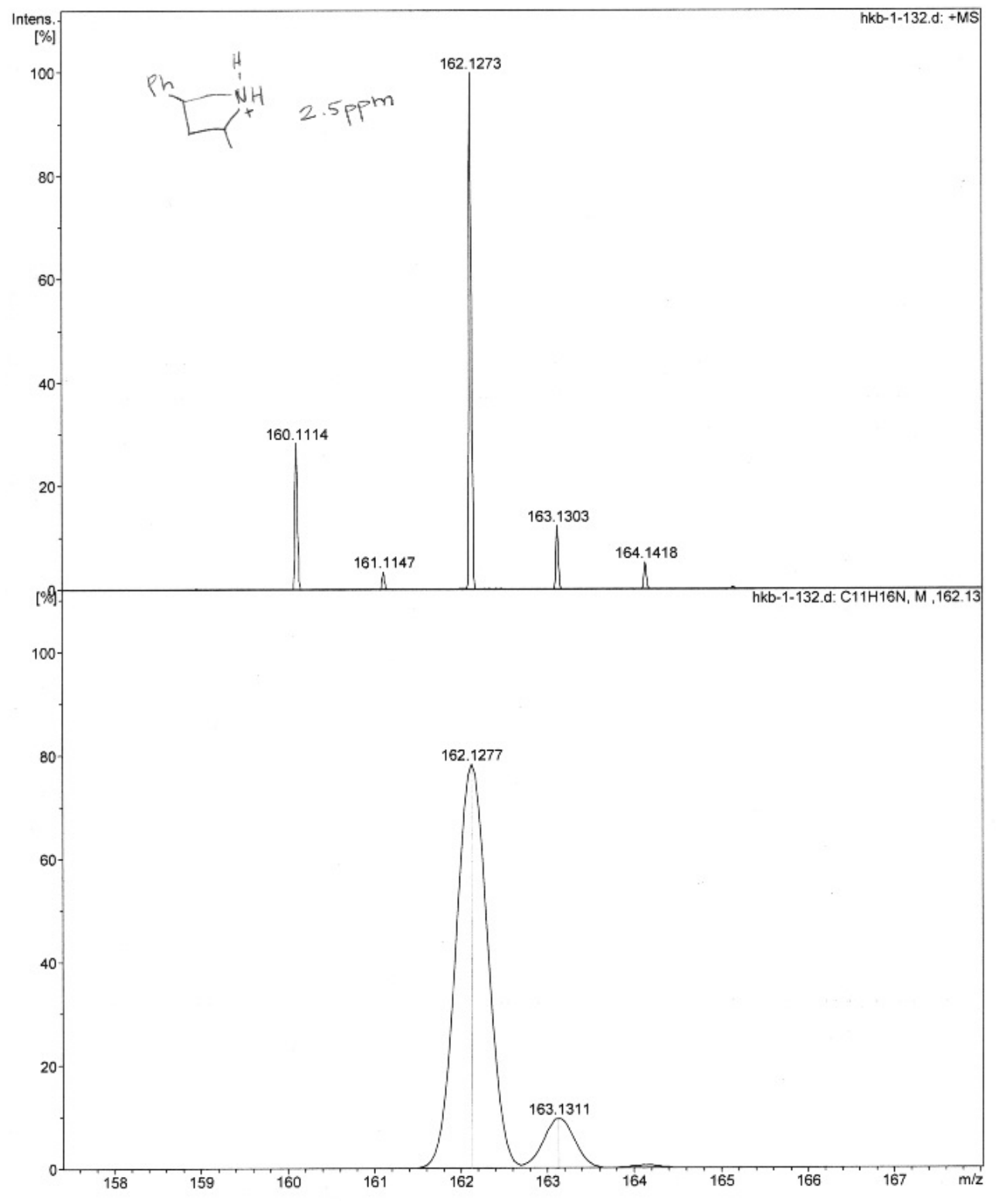

Figure S51. HRMS spectrum of catalytic trial (Entry 8, HA product)

HRMS (ESI) $m / z$ calculated for $\mathrm{C}_{11} \mathrm{H}_{16} \mathrm{~N}[\mathrm{M}+\mathrm{H}]^{+} 162.1277$ (bottom), found 162.1273 (top) 


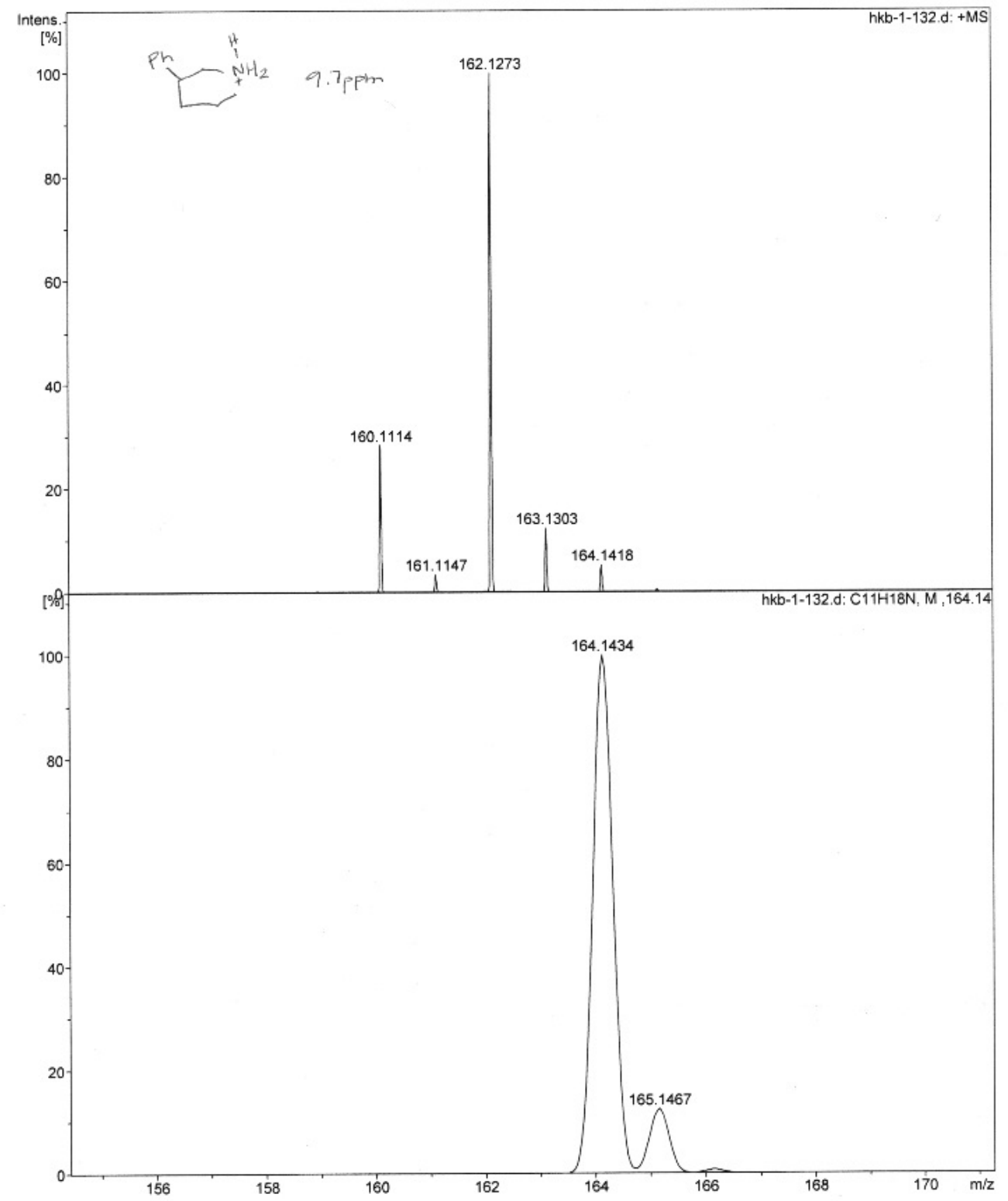

Figure S52. HRMS spectrum of catalytic trial (Entry 8, saturated product) HRMS (ESI) $m / z$, calculated for $\mathrm{C}_{11} \mathrm{H}_{18} \mathrm{~N}[\mathrm{M}+\mathrm{H}]^{+} 164.1434$ (bottom), found 164.1418 (top) 
X-ray Crystallographic Data of (1,3-bis(3-butylimidazol-1-yl-2-idene)-2-phenylene)bis(tbutylimido)tantalum( $(V)$ lithium bridged dimer (2)

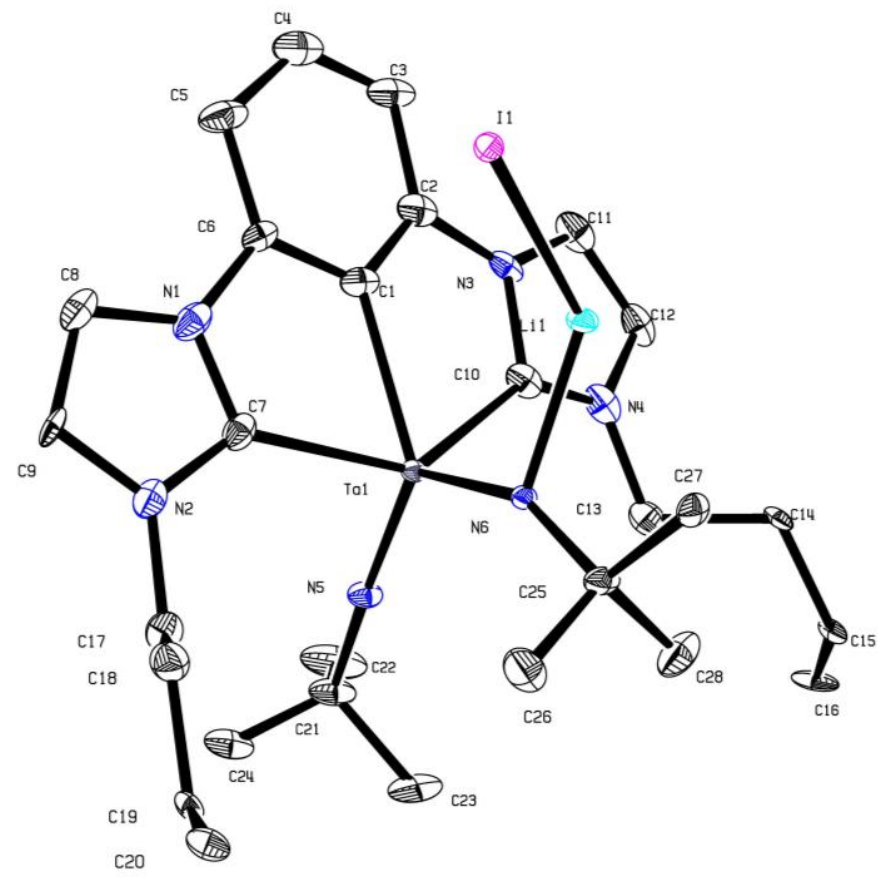

Figure S53. ORTEP of the asymmetric unit of 2. 
Table S3. Crystal data and structure refinement for $\mathbf{2}$.

Identification code

Empirical formula

Formula weight

Temperature

Wavelength

Crystal system

Space group

Unit cell dimensions

Volume

$\mathrm{Z}$

Density (calculated)

Absorption coefficient

$\mathrm{F}(000)$

Crystal size

Theta range for data collection

Index ranges

Reflections collected

Independent reflections

Completeness to theta $=25.242^{\circ}$

Absorption correction

Max. and min. transmission

Refinement method

Data / restraints / parameters

Goodness-of-fit on $\mathrm{F}^{2}$

Final $\mathrm{R}$ indices [I $>2 \operatorname{sigma}(\mathrm{I})]$

$\mathrm{R}$ indices (all data)

Extinction coefficient

Largest diff. peak and hole tri

C28 H43 I Li N6 Ta

778.47

$100.15 \mathrm{~K}$

$0.71073 \AA$

Triclinic

P-1

$\mathrm{a}=10.4676(5) \AA$

$\alpha=96.432(2)^{\circ}$.

$\mathrm{b}=11.0982(5) \AA$

$\beta=92.191(2)^{\circ}$.

$\mathrm{c}=15.7331(8) \AA$

$\gamma=117.377(2)^{\circ}$.
2

$1.611 \mathrm{Mg} / \mathrm{m}^{3}$

$4.413 \mathrm{~mm}^{-1}$

764

$0.3 \times 0.3 \times 0.05 \mathrm{~mm}^{3}$

2.089 to $28.281^{\circ}$.

$-13<=\mathrm{h}<=13,-14<=\mathrm{k}<=14,-20<=\mathrm{l}<=20$

39862

$7885[\mathrm{R}(\mathrm{int})=0.0499]$

$100.0 \%$

Semi-empirical from equivalents

0.7456 and 0.4746

Full-matrix least-squares on $\mathrm{F}^{2}$

7885 / 30 / 355

1.057

$\mathrm{R} 1=0.0448, \mathrm{wR} 2=0.1014$

$\mathrm{R} 1=0.0558, \mathrm{wR} 2=0.1073$

$\mathrm{n} / \mathrm{a}$

1.739 and -2.022 e. $\AA^{-3}$ 
Table S4. Fractional Atomic Coordinates $\left(\times 10^{4}\right)$ and Equivalent Isotropic Displacement Parameters $\left(\AA^{2} \times 10^{3}\right)$ for 2 . $U_{\text {eq }}$ is defined as $1 / 3$ of of the trace of the orthogonalised $U_{\text {IJ }}$ tensor. Atom

$$
\mathrm{X}
$$$$
\mathrm{y}
$$

$\mathrm{Z}$

$\mathrm{U}(\mathrm{eq})$

\begin{tabular}{|c|c|c|c|c|}
\hline $\mathrm{Ta}(1)$ & 7396(1) & $7781(1)$ & 7041(1) & $24(1)$ \\
\hline $\mathrm{I}(1)$ & $9068(1)$ & $11406(1)$ & 5164(1) & $30(1)$ \\
\hline $\mathrm{N}(1)$ & $8350(9)$ & $5504(7)$ & $6085(6)$ & $69(2)$ \\
\hline $\mathrm{N}(2)$ & $7170(6)$ & 6054(6) & $5243(4)$ & $39(1)$ \\
\hline $\mathrm{N}(3)$ & $5251(7)$ & $8865(7)$ & $6373(6)$ & $61(2)$ \\
\hline $\mathrm{N}(4)$ & $5602(7)$ & $9616(7)$ & 7704(5) & $50(2)$ \\
\hline $\mathrm{N}(5)$ & $6739(6)$ & $6849(6)$ & 7928(4) & $39(1)$ \\
\hline $\mathrm{N}(6)$ & $9248(5)$ & $9250(5)$ & $7220(3)$ & $24(1)$ \\
\hline $\mathrm{C}(1)$ & $11190(20)$ & 4571(14) & $7856(8)$ & 131(7) \\
\hline$C(2)$ & $11676(14)$ & $5908(14)$ & $7548(7)$ & $86(4)$ \\
\hline $\mathrm{C}(3)$ & $10728(14)$ & $5809(13)$ & $6745(7)$ & $81(3)$ \\
\hline $\mathrm{C}(4)$ & $9114(10)$ & $5438(9)$ & 6894(6) & $59(2)$ \\
\hline $\mathrm{C}(5)$ & $8267(10)$ & $4758(8)$ & $5348(7)$ & $63(3)$ \\
\hline$C(6)$ & $7455(11)$ & $5120(10)$ & $4763(7)$ & $71(3)$ \\
\hline$C(7)$ & 7751(9) & $6300(8)$ & $6109(6)$ & $52(2)$ \\
\hline $\mathrm{C}(8)$ & 6352(9) & $6760(10)$ & $5032(5)$ & $67(3)$ \\
\hline $\mathrm{C}(9)$ & $5737(10)$ & $6579(12)$ & $4184(6)$ & $86(4)$ \\
\hline$C(10)$ & $4958(11)$ & $7269(12)$ & $4088(6)$ & $83(4)$ \\
\hline $\mathrm{C}(11)$ & $4718(10)$ & $8070(12)$ & 4792(7) & $80(4)$ \\
\hline$C(12)$ & $5405(7)$ & $8152(7)$ & $5602(5)$ & $36(2)$ \\
\hline$C(13)$ & $6262(8)$ & $7475(8)$ & $5722(5)$ & $54(2)$ \\
\hline$C(14)$ & 4391(9) & $9466(9)$ & 6464(7) & $61(3)$ \\
\hline$C(15)$ & $4662(11)$ & $9976(11)$ & $7363(8)$ & $76(3)$ \\
\hline$C(16)$ & $5995(8)$ & $8891(8)$ & 7074(6) & $51(2)$ \\
\hline$C(17)$ & $6189(10)$ & $9935(10)$ & $8603(6)$ & $59(2)$ \\
\hline $\mathrm{C}(18)$ & $7061(10)$ & 11461(9) & $8866(6)$ & $57(2)$ \\
\hline $\mathrm{C}(19)$ & $7716(14)$ & 11794(11) & 9795(7) & $77(3)$ \\
\hline$C(20)$ & $8674(17)$ & $13310(13)$ & 10050(9) & $107(5)$ \\
\hline $\mathrm{C}(21)$ & $6282(8)$ & $6089(7)$ & $8650(5)$ & $57(2)$ \\
\hline $\mathrm{C}(22)$ & $5358(15)$ & $4579(10)$ & $8326(8)$ & $62(4)$ \\
\hline$C(22 \mathrm{~A})$ & $6240(20)$ & $4698(16)$ & $8455(15)$ & $62(4)$ \\
\hline $\mathrm{C}(23)$ & $7651(13)$ & $6261(14)$ & 9193(9) & $66(4)$ \\
\hline
\end{tabular}




$\begin{array}{lcclr}\mathrm{C}(23 \mathrm{~A}) & 7190(20) & 6900(20) & 9474(12) & 66(4) \\ \mathrm{C}(24) & 5487(16) & 6647(14) & 9215(9) & 70(4) \\ \mathrm{C}(24 \mathrm{~A}) & 4715(17) & 5840(20) & 8713(16) & 70(4) \\ \mathrm{C}(25) & 10583(8) & 10132(8) & 7768(4) & 42(2) \\ \mathrm{C}(26) & 10295(10) & 10870(13) & 8501(7) & 101(5) \\ \mathrm{C}(27) & 11721(7) & 11119(7) & 7266(5) & 38(2) \\ \mathrm{C}(28) & 11194(10) & 9228(11) & 8117(8) & 87(4) \\ \mathrm{Li}(1) & 9622(12) & 9645(12) & 5973(7) & 35(2)\end{array}$


Table S5. Anisotropic Displacement Parameters $\left(\AA^{2} \times 10^{3}\right)$ for 2. The Anisotropic displacement factor exponent takes the form: $-2 \pi^{2}\left[\mathrm{~h}^{2} \mathrm{a} * 2 \mathrm{U}_{11}+2 \mathrm{hka} * \mathrm{~b} * \mathrm{U}_{12}+\ldots\right]$.

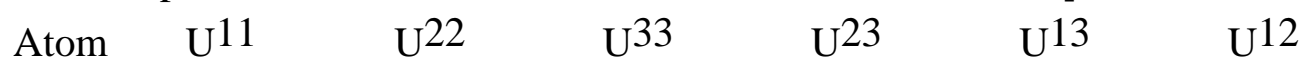

\begin{tabular}{|c|c|c|c|c|c|c|}
\hline $\mathrm{Ta}(1)$ & $22(1)$ & $24(1)$ & $22(1)$ & $6(1)$ & $3(1)$ & $6(1)$ \\
\hline $\mathrm{I}(1)$ & $28(1)$ & $31(1)$ & $31(1)$ & $9(1)$ & $4(1)$ & $13(1)$ \\
\hline $\mathrm{N}(1)$ & $64(5)$ & $35(4)$ & $80(6)$ & $-9(3)$ & $38(4)$ & $1(3)$ \\
\hline $\mathrm{N}(2)$ & $33(3)$ & $31(3)$ & $28(3)$ & $-3(2)$ & $7(2)$ & $-6(2)$ \\
\hline $\mathrm{N}(3)$ & $31(3)$ & $49(4)$ & $92(6)$ & $35(4)$ & $-8(3)$ & $4(3)$ \\
\hline $\mathrm{N}(4)$ & $41(3)$ & $51(4)$ & $60(4)$ & $15(3)$ & $2(3)$ & $20(3)$ \\
\hline $\mathrm{N}(5)$ & $37(3)$ & $34(3)$ & $34(3)$ & $12(2)$ & $3(2)$ & $4(2)$ \\
\hline $\mathrm{N}(6)$ & $24(2)$ & $28(2)$ & $19(2)$ & $4(2)$ & $2(2)$ & $10(2)$ \\
\hline $\mathrm{C}(1)$ & $280(20)$ & $116(11)$ & $57(7)$ & $14(7)$ & $8(10)$ & $148(14)$ \\
\hline$C(2)$ & 114(10) & $124(10)$ & $72(7)$ & $33(7)$ & $30(7)$ & $96(9)$ \\
\hline$C(3)$ & 115(9) & 113(9) & $66(6)$ & $33(6)$ & $30(6)$ & $91(8)$ \\
\hline$C(4)$ & $71(6)$ & $54(5)$ & $48(5)$ & $24(4)$ & $13(4)$ & $23(4)$ \\
\hline$C(5)$ & $50(5)$ & $35(4)$ & $83(7)$ & $-14(4)$ & $31(5)$ & $6(4)$ \\
\hline$C(6)$ & $60(6)$ & $56(5)$ & $63(6)$ & $-14(5)$ & $19(5)$ & $3(5)$ \\
\hline$C(7)$ & $45(4)$ & $31(4)$ & $59(5)$ & $3(3)$ & $17(4)$ & $1(3)$ \\
\hline$C(8)$ & $41(4)$ & $63(5)$ & $39(4)$ & $19(4)$ & $-5(3)$ & $-26(4)$ \\
\hline $\mathrm{C}(9)$ & $44(5)$ & $94(7)$ & $42(5)$ & $41(5)$ & $-16(4)$ & $-37(5)$ \\
\hline$C(10)$ & $56(6)$ & $92(8)$ & $42(5)$ & $21(5)$ & $-14(4)$ & $-17(6)$ \\
\hline$C(11)$ & $47(5)$ & $91(8)$ & $64(6)$ & $40(6)$ & $-15(4)$ & $-4(5)$ \\
\hline$C(12)$ & $22(3)$ & $31(3)$ & $40(4)$ & $13(3)$ & $-6(3)$ & $-2(2)$ \\
\hline$C(13)$ & $41(4)$ & $43(4)$ & $33(4)$ & $12(3)$ & $-6(3)$ & $-19(3)$ \\
\hline $\mathrm{C}(14)$ & $36(4)$ & $55(5)$ & $99(8)$ & $33(5)$ & $-4(4)$ & $21(4)$ \\
\hline$C(15)$ & $57(6)$ & $80(7)$ & 101(9) & $13(6)$ & $-11(6)$ & $42(5)$ \\
\hline$C(16)$ & $29(4)$ & $47(4)$ & $75(6)$ & $28(4)$ & $5(4)$ & $13(3)$ \\
\hline$C(17)$ & $61(5)$ & $68(6)$ & $62(6)$ & $19(5)$ & $16(4)$ & $39(5)$ \\
\hline $\mathrm{C}(18)$ & $67(6)$ & $60(5)$ & $56(5)$ & $7(4)$ & $12(4)$ & $39(5)$ \\
\hline$C(19)$ & 107(9) & $77(7)$ & $57(6)$ & $3(5)$ & $22(6)$ & $51(7)$ \\
\hline$C(20)$ & 145(13) & $80(8)$ & $81(9)$ & $-11(7)$ & $10(8)$ & $45(9)$ \\
\hline $\mathrm{C}(21)$ & $59(5)$ & $47(4)$ & $42(4)$ & $22(4)$ & $4(4)$ & $3(4)$ \\
\hline$C(22)$ & $69(10)$ & $33(5)$ & $42(6)$ & $16(4)$ & $1(7)$ & $-14(7)$ \\
\hline $\mathrm{C}(22 \mathrm{~A})$ & $69(10)$ & $33(5)$ & $42(6)$ & $16(4)$ & $1(7)$ & $-14(7)$ \\
\hline$C(23)$ & $77(10)$ & $51(8)$ & $46(7)$ & $19(6)$ & $3(6)$ & $7(6)$ \\
\hline
\end{tabular}




$\begin{array}{lcccccc}\mathrm{C}(23 \mathrm{~A}) & 77(10) & 51(8) & 46(7) & 19(6) & 3(6) & 7(6) \\ \mathrm{C}(24) & 75(10) & 56(8) & 57(8) & 10(6) & 24(7) & 10(7) \\ \mathrm{C}(24 \mathrm{~A}) & 75(10) & 56(8) & 57(8) & 10(6) & 24(7) & 10(7) \\ \mathrm{C}(25) & 31(3) & 45(4) & 30(3) & 6(3) & -1(3) & 1(3) \\ \mathrm{C}(26) & 44(5) & 126(10) & 59(6) & -54(6) & 8(4) & -8(6) \\ \mathrm{C}(27) & 24(3) & 33(3) & 43(4) & 6(3) & -2(3) & 2(3) \\ \mathrm{C}(28) & 40(5) & 81(7) & 125(10) & 65(7) & -20(5) & 6(5) \\ \mathrm{Li}(1) & 37(6) & 40(6) & 27(5) & 17(5) & 9(4) & 15(5)\end{array}$


Table S6. Bond Lengths for 2.

\begin{tabular}{|c|c|c|c|}
\hline $\mathrm{Ta}(1)-\mathrm{N}(5)$ & $1.799(5)$ & $\mathrm{C}(5)-\mathrm{H}(5)$ & 0.9500 \\
\hline $\mathrm{Ta}(1)-\mathrm{N}(6)$ & $1.856(5)$ & $C(5)-C(6)$ & $1.438(16)$ \\
\hline $\mathrm{Ta}(1)-\mathrm{C}(7)$ & $2.248(8)$ & $\mathrm{C}(6)-\mathrm{H}(6)$ & 0.9500 \\
\hline $\mathrm{Ta}(1)-\mathrm{C}(13)$ & $2.260(7)$ & $C(8)-C(9)$ & $1.411(11)$ \\
\hline $\mathrm{Ta}(1)-\mathrm{C}(16)$ & $2.307(8)$ & $\mathrm{C}(8)-\mathrm{C}(13)$ & $1.305(13)$ \\
\hline $\mathrm{Ta}(1)-\mathrm{Li}(1)$ & $3.029(11)$ & $\mathrm{C}(9)-\mathrm{H}(9)$ & 0.9500 \\
\hline $\mathrm{I}(1)-\mathrm{Li}(1)$ & $2.705(11)$ & $\mathrm{C}(9)-\mathrm{C}(10)$ & $1.365(18)$ \\
\hline $\mathrm{I}(1)-\mathrm{Li}(1) \# 1$ & $2.762(12)$ & $\mathrm{C}(10)-\mathrm{H}(10)$ & 0.9500 \\
\hline $\mathrm{N}(1)-\mathrm{C}(4)$ & $1.504(13)$ & $C(10)-C(11)$ & $1.444(18)$ \\
\hline $\mathrm{N}(1)-\mathrm{C}(5)$ & $1.323(11)$ & $\mathrm{C}(11)-\mathrm{H}(11)$ & 0.9500 \\
\hline $\mathrm{N}(1)-\mathrm{C}(7)$ & $1.296(12)$ & $C(11)-C(12)$ & $1.413(11)$ \\
\hline $\mathrm{N}(2)-\mathrm{C}(6)$ & $1.363(11)$ & $C(12)-C(13)$ & $1.429(12)$ \\
\hline$N(2)-C(7)$ & $1.414(11)$ & $\mathrm{C}(14)-\mathrm{H}(14)$ & 0.9500 \\
\hline $\mathrm{N}(2)-\mathrm{C}(8)$ & $1.453(13)$ & $C(14)-C(15)$ & $1.429(15)$ \\
\hline $\mathrm{N}(3)-\mathrm{C}(12)$ & $1.428(11)$ & $\mathrm{C}(15)-\mathrm{H}(15)$ & 0.9500 \\
\hline $\mathrm{N}(3)-\mathrm{C}(14)$ & $1.348(11)$ & $\mathrm{C}(17)-\mathrm{H}(17 \mathrm{~A})$ & 0.9900 \\
\hline $\mathrm{N}(3)-\mathrm{C}(16)$ & $1.316(11)$ & $\mathrm{C}(17)-\mathrm{H}(17 \mathrm{~B})$ & 0.9900 \\
\hline $\mathrm{N}(4)-\mathrm{C}(15)$ & $1.336(11)$ & $C(17)-C(18)$ & $1.504(13)$ \\
\hline $\mathrm{N}(4)-\mathrm{C}(16)$ & $1.398(11)$ & $\mathrm{C}(18)-\mathrm{H}(18 \mathrm{~A})$ & 0.9900 \\
\hline $\mathrm{N}(4)-\mathrm{C}(17)$ & $1.458(11)$ & $\mathrm{C}(18)-\mathrm{H}(18 \mathrm{~B})$ & 0.9900 \\
\hline $\mathrm{N}(5)-\mathrm{C}(21)$ & $1.455(9)$ & $\mathrm{C}(18)-\mathrm{C}(19)$ & $1.521(14)$ \\
\hline $\mathrm{N}(6)-\mathrm{C}(25)$ & $1.454(8)$ & $\mathrm{C}(19)-\mathrm{H}(19 \mathrm{~A})$ & 0.9900 \\
\hline $\mathrm{N}(6)-\operatorname{Li}(1)$ & $2.071(11)$ & $\mathrm{C}(19)-\mathrm{H}(19 \mathrm{~B})$ & 0.9900 \\
\hline $\mathrm{C}(1)-\mathrm{H}(1 \mathrm{~A})$ & 0.9800 & $C(19)-C(20)$ & $1.504(16)$ \\
\hline $\mathrm{C}(1)-\mathrm{H}(1 \mathrm{~B})$ & 0.9800 & $\mathrm{C}(20)-\mathrm{H}(20 \mathrm{~A})$ & 0.9800 \\
\hline $\mathrm{C}(1)-\mathrm{H}(1 \mathrm{C})$ & 0.9800 & $\mathrm{C}(20)-\mathrm{H}(20 \mathrm{~B})$ & 0.9800 \\
\hline$C(1)-C(2)$ & $1.477(16)$ & $\mathrm{C}(20)-\mathrm{H}(20 \mathrm{C})$ & 0.9800 \\
\hline $\mathrm{C}(2)-\mathrm{H}(2 \mathrm{~A})$ & 0.9900 & $C(21)-C(22)$ & $1.512(11)$ \\
\hline $\mathrm{C}(2)-\mathrm{H}(2 \mathrm{~B})$ & 0.9900 & $\mathrm{C}(21)-\mathrm{C}(22 \mathrm{~A})$ & $1.522(13)$ \\
\hline$C(2)-C(3)$ & $1.538(15)$ & $C(21)-C(23)$ & $1.561(12)$ \\
\hline $\mathrm{C}(3)-\mathrm{H}(3 \mathrm{~A})$ & 0.9900 & $C(21)-C(23 A)$ & $1.500(13)$ \\
\hline $\mathrm{C}(3)-\mathrm{H}(3 \mathrm{~B})$ & 0.9900 & $\mathrm{C}(21)-\mathrm{C}(24)$ & $1.507(12)$ \\
\hline$C(3)-C(4)$ & $1.578(14)$ & $\mathrm{C}(21)-\mathrm{C}(24 \mathrm{~A})$ & $1.542(14)$ \\
\hline $\mathrm{C}(4)-\mathrm{H}(4 \mathrm{~A})$ & 0.9900 & $\mathrm{C}(22)-\mathrm{H}(22 \mathrm{~A})$ & 0.9800 \\
\hline $\mathrm{C}(4)-\mathrm{H}(4 \mathrm{~B})$ & 0.9900 & $\mathrm{C}(22)-\mathrm{H}(22 \mathrm{~B})$ & 0.9800 \\
\hline
\end{tabular}




$\begin{array}{llll}\mathrm{C}(22)-\mathrm{H}(22 \mathrm{C}) & 0.9800 & \mathrm{C}(24 \mathrm{~A})-\mathrm{H}(24 \mathrm{~F}) & 0.9800 \\ \mathrm{C}(22 \mathrm{~A})-\mathrm{H}(22 \mathrm{D}) & 0.9800 & \mathrm{C}(25)-\mathrm{C}(26) & 1.461(12) \\ \mathrm{C}(22 \mathrm{~A})-\mathrm{H}(22 \mathrm{E}) & 0.9800 & \mathrm{C}(25)-\mathrm{C}(27) & 1.519(9) \\ \mathrm{C}(22 \mathrm{~A})-\mathrm{H}(22 \mathrm{~F}) & 0.9800 & \mathrm{C}(25)-\mathrm{C}(28) & 1.551(12) \\ \mathrm{C}(23)-\mathrm{H}(23 \mathrm{~A}) & 0.9800 & \mathrm{C}(26)-\mathrm{H}(26 \mathrm{~A}) & 0.9800 \\ \mathrm{C}(23)-\mathrm{H}(23 \mathrm{~B}) & 0.9800 & \mathrm{C}(26)-\mathrm{H}(26 \mathrm{~B}) & 0.9800 \\ \mathrm{C}(23)-\mathrm{H}(23 \mathrm{C}) & 0.9800 & \mathrm{C}(26)-\mathrm{H}(26 \mathrm{C}) & 0.9800 \\ \mathrm{C}(23 \mathrm{~A})-\mathrm{H}(23 \mathrm{D}) & 0.9800 & \mathrm{C}(27)-\mathrm{H}(27 \mathrm{~A}) & 0.9800 \\ \mathrm{C}(23 \mathrm{~A})-\mathrm{H}(23 \mathrm{E}) & 0.9800 & \mathrm{C}(27)-\mathrm{H}(27 \mathrm{~B}) & 0.9800 \\ \mathrm{C}(23 \mathrm{~A})-\mathrm{H}(23 \mathrm{~F}) & 0.9800 & \mathrm{C}(27)-\mathrm{H}(27 \mathrm{C}) & 0.9800 \\ \mathrm{C}(24)-\mathrm{H}(24 \mathrm{~A}) & 0.9800 & \mathrm{C}(28)-\mathrm{H}(28 \mathrm{~A}) & 0.9800 \\ \mathrm{C}(24)-\mathrm{H}(24 \mathrm{~B}) & 0.9800 & \mathrm{C}(28)-\mathrm{H}(28 \mathrm{~B}) & 0.9800 \\ \mathrm{C}(24)-\mathrm{H}(24 \mathrm{C}) & 0.9800 & \mathrm{C}(28)-\mathrm{H}(28 \mathrm{C}) & 0.9800 \\ \mathrm{C}(24 \mathrm{~A})-\mathrm{H}(24 \mathrm{D}) & 0.9800 & \mathrm{Li}(1)-\mathrm{I}(1) \# 1 & 2.762(12) \\ \mathrm{C}(24 \mathrm{~A})-\mathrm{H}(24 \mathrm{E}) & 0.9800 & \mathrm{Li}(1)-\mathrm{Li}(1) \# 1 & 3.28(2)\end{array}$

Symmetry transformations used to generate equivalent atoms: $\# 1-x+2,-y+2,-z+1$ 
Table S7. Bond Angles for 2.

$\begin{array}{lc}\mathrm{N}(5)-\mathrm{Ta}(1)-\mathrm{N}(6) & 116.8(2) \\ \mathrm{N}(5)-\mathrm{Ta}(1)-\mathrm{C}(7) & 103.1(3) \\ \mathrm{N}(5)-\mathrm{Ta}(1)-\mathrm{C}(13) & 129.4(3) \\ \mathrm{N}(5)-\mathrm{Ta}(1)-\mathrm{C}(16) & 98.4(3) \\ \mathrm{N}(5)-\mathrm{Ta}(1)-\mathrm{Li}(1) & 156.9(3) \\ \mathrm{N}(6)-\mathrm{Ta}(1)-\mathrm{C}(7) & 100.5(2) \\ \mathrm{N}(6)-\mathrm{Ta}(1)-\mathrm{C}(13) & 113.8(2) \\ \mathrm{N}(6)-\mathrm{Ta}(1)-\mathrm{C}(16) & 101.6(2) \\ \mathrm{N}(6)-\mathrm{Ta}(1)-\mathrm{Li}(1) & 42.2(3) \\ \mathrm{C}(7)-\mathrm{Ta}(1)-\mathrm{C}(13) & 68.7(4) \\ \mathrm{C}(7)-\mathrm{Ta}(1)-\mathrm{C}(16) & 137.7(3) \\ \mathrm{C}(7)-\mathrm{Ta}(1)-\mathrm{Li}(1) & 77.1(3) \\ \mathrm{C}(13)-\mathrm{Ta}(1)-\mathrm{C}(16) & 69.4(4) \\ \mathrm{C}(13)-\mathrm{Ta}(1)-\mathrm{Li}(1) & 72.7(3) \\ \mathrm{C}(16)-\mathrm{Ta}(1)-\mathrm{Li}(1) & 96.3(3) \\ \mathrm{Li}(1)-\mathrm{I}(1)-\mathrm{Li}(1) \# 1 & 73.7(3) \\ \mathrm{C}(5)-\mathrm{N}(1)-\mathrm{C}(4) & 121.3(9) \\ \mathrm{C}(7)-\mathrm{N}(1)-\mathrm{C}(4) & 119.6(8) \\ \mathrm{C}(7)-\mathrm{N}(1)-\mathrm{C}(5) & 119.1(10) \\ \mathrm{C}(6)-\mathrm{N}(2)-\mathrm{C}(7) & 111.1(8) \\ \mathrm{C}(6)-\mathrm{N}(2)-\mathrm{C}(8) & 132.0(8) \\ \mathrm{C}(7)-\mathrm{N}(2)-\mathrm{C}(8) & 116.9(7) \\ \mathrm{C}(14)-\mathrm{N}(3)-\mathrm{C}(12) & 127.2(8) \\ \mathrm{C}(16)-\mathrm{N}(3)-\mathrm{C}(12) & 115.9(7) \\ \mathrm{C}(16)-\mathrm{N}(3)-\mathrm{C}(14) & 116.8(9) \\ \mathrm{C}(15)-\mathrm{N}(4)-\mathrm{C}(16) & 111.0(8) \\ \mathrm{C}(15)-\mathrm{N}(4)-\mathrm{C}(17) & 126.2(9) \\ \mathrm{C}(16)-\mathrm{N}(4)-\mathrm{C}(17) & 122.9(7) \\ \mathrm{C}(21)-\mathrm{N}(5)-\mathrm{Ta}(1) & 176.8(6) \\ \mathrm{Ta}(1)-\mathrm{N}(6)-\mathrm{Li}(1) & 100.8(4) \\ \mathrm{C}(25)-\mathrm{N}(6)-\mathrm{Ta}(1) & 151.0(4) \\ \mathrm{C}(25)-\mathrm{N}(6)-\mathrm{Li}(1) & 107.6(5) \\ \mathrm{H}(1 \mathrm{~A})-\mathrm{C}(1)-\mathrm{H}(1 \mathrm{~B}) & 109.5 \\ \mathrm{H}(1 \mathrm{~A})-\mathrm{C}(1)-\mathrm{H}(1 \mathrm{C}) & 109.5 \\ \mathrm{H}(1 \mathrm{~B})-\mathrm{C}(1)-\mathrm{H}(1 \mathrm{C}) & 109.5 \\ & \end{array}$

$\begin{array}{ll}\mathrm{C}(2)-\mathrm{C}(1)-\mathrm{H}(1 \mathrm{~A}) & 109.5 \\ \mathrm{C}(2)-\mathrm{C}(1)-\mathrm{H}(1 \mathrm{~B}) & 109.5 \\ \mathrm{C}(2)-\mathrm{C}(1)-\mathrm{H}(1 \mathrm{C}) & 109.5 \\ \mathrm{C}(1)-\mathrm{C}(2)-\mathrm{H}(2 \mathrm{~A}) & 109.2 \\ \mathrm{C}(1)-\mathrm{C}(2)-\mathrm{H}(2 \mathrm{~B}) & 109.2 \\ \mathrm{C}(1)-\mathrm{C}(2)-\mathrm{C}(3) & 112.1(12) \\ \mathrm{H}(2 \mathrm{~A})-\mathrm{C}(2)-\mathrm{H}(2 \mathrm{~B}) & 107.9 \\ \mathrm{C}(3)-\mathrm{C}(2)-\mathrm{H}(2 \mathrm{~A}) & 109.2 \\ \mathrm{C}(3)-\mathrm{C}(2)-\mathrm{H}(2 \mathrm{~B}) & 109.2 \\ \mathrm{C}(2)-\mathrm{C}(3)-\mathrm{H}(3 \mathrm{~A}) & 108.5 \\ \mathrm{C}(2)-\mathrm{C}(3)-\mathrm{H}(3 \mathrm{~B}) & 108.5 \\ \mathrm{C}(2)-\mathrm{C}(3)-\mathrm{C}(4) & 114.9(9) \\ \mathrm{H}(3 \mathrm{~A})-\mathrm{C}(3)-\mathrm{H}(3 \mathrm{~B}) & 107.5 \\ \mathrm{C}(4)-\mathrm{C}(3)-\mathrm{H}(3 \mathrm{~A}) & 108.5 \\ \mathrm{C}(4)-\mathrm{C}(3)-\mathrm{H}(3 \mathrm{~B}) & 108.5 \\ \mathrm{~N}(1)-\mathrm{C}(4)-\mathrm{C}(3) & 109.5(7) \\ \mathrm{N}(1)-\mathrm{C}(4)-\mathrm{H}(4 \mathrm{~A}) & 109.8 \\ \mathrm{~N}(1)-\mathrm{C}(4)-\mathrm{H}(4 \mathrm{~B}) & 109.8 \\ \mathrm{C}(3)-\mathrm{C}(4)-\mathrm{H}(4 \mathrm{~A}) & 109.8 \\ \mathrm{C}(3)-\mathrm{C}(4)-\mathrm{H}(4 \mathrm{~B}) & 109.8 \\ \mathrm{H}(4 \mathrm{~A})-\mathrm{C}(4)-\mathrm{H}(4 \mathrm{~B}) & 108.2 \\ \mathrm{~N}(1)-\mathrm{C}(5)-\mathrm{H}(5) & 128.5 \\ \mathrm{~N}(1)-\mathrm{C}(5)-\mathrm{C}(6) & 102.9(9) \\ \mathrm{C}(6)-\mathrm{C}(5)-\mathrm{H}(5) & 128.5 \\ \mathrm{~N}(2)-\mathrm{C}(6)-\mathrm{C}(5) & 105.5(8) \\ \mathrm{N}(2)-\mathrm{C}(6)-\mathrm{H}(6) & 127.3 \\ \mathrm{C}(5)-\mathrm{C}(6)-\mathrm{H}(6) & 127.3 \\ \mathrm{~N}(1)-\mathrm{C}(7)-\mathrm{Ta}(1) & 140.5(7) \\ \mathrm{N}(1)-\mathrm{C}(7)-\mathrm{N}(2) & 101.4(7) \\ \mathrm{N}(2)-\mathrm{C}(7)-\mathrm{Ta}(1) & 118.1(6) \\ \mathrm{C}(9)-\mathrm{C}(8)-\mathrm{N}(2) & 120.8(11) \\ \mathrm{C}(13)-\mathrm{C}(8)-\mathrm{N}(2) & 110.3(7) \\ \mathrm{C}(13)-\mathrm{C}(8)-\mathrm{C}(9) & 128.9(12) \\ \mathrm{C}(8)-\mathrm{C}(9)-\mathrm{H}(9) & 122.9 \\ \mathrm{C}(10)-\mathrm{C}(9)-\mathrm{C}(8) & 114.2(12) \\ & \end{array}$




\begin{tabular}{|c|c|c|c|}
\hline $\mathrm{C}(10)-\mathrm{C}(9)-\mathrm{H}(9)$ & 122.9 & $\mathrm{H}(19 \mathrm{~A})-\mathrm{C}(19)-\mathrm{H}(19 \mathrm{~B})$ & 108.0 \\
\hline $\mathrm{C}(9)-\mathrm{C}(10)-\mathrm{H}(10)$ & 118.1 & $\mathrm{C}(20)-\mathrm{C}(19)-\mathrm{C}(18)$ & $111.5(10)$ \\
\hline $\mathrm{C}(9)-\mathrm{C}(10)-\mathrm{C}(11)$ & 123.7(10) & $\mathrm{C}(20)-\mathrm{C}(19)-\mathrm{H}(19 \mathrm{~A})$ & 109.3 \\
\hline $\mathrm{C}(11)-\mathrm{C}(10)-\mathrm{H}(10)$ & 118.1 & $\mathrm{C}(20)-\mathrm{C}(19)-\mathrm{H}(19 \mathrm{~B})$ & 109.3 \\
\hline $\mathrm{C}(10)-\mathrm{C}(11)-\mathrm{H}(11)$ & 122.4 & $\mathrm{C}(19)-\mathrm{C}(20)-\mathrm{H}(20 \mathrm{~A})$ & 109.5 \\
\hline $\mathrm{C}(12)-\mathrm{C}(11)-\mathrm{C}(10)$ & $115.3(11)$ & $\mathrm{C}(19)-\mathrm{C}(20)-\mathrm{H}(20 \mathrm{~B})$ & 109.5 \\
\hline $\mathrm{C}(12)-\mathrm{C}(11)-\mathrm{H}(11)$ & 122.4 & $\mathrm{C}(19)-\mathrm{C}(20)-\mathrm{H}(20 \mathrm{C})$ & 109.5 \\
\hline $\mathrm{N}(3)-\mathrm{C}(12)-\mathrm{C}(13)$ & $114.6(6)$ & $\mathrm{H}(20 \mathrm{~A})-\mathrm{C}(20)-\mathrm{H}(20 \mathrm{~B})$ & 109.5 \\
\hline C(11)-C(12)-N(3) & $122.9(8)$ & $H(20 A)-C(20)-H(20 C)$ & 109.5 \\
\hline $\mathrm{C}(11)-\mathrm{C}(12)-\mathrm{C}(13)$ & $122.5(9)$ & $\mathrm{H}(20 \mathrm{~B})-\mathrm{C}(20)-\mathrm{H}(20 \mathrm{C})$ & 109.5 \\
\hline $\mathrm{C}(8)-\mathrm{C}(13)-\mathrm{Ta}(1)$ & $125.8(8)$ & $\mathrm{N}(5)-\mathrm{C}(21)-\mathrm{C}(22)$ & $109.9(7)$ \\
\hline $\mathrm{C}(8)-\mathrm{C}(13)-\mathrm{C}(12)$ & $115.3(8)$ & $\mathrm{N}(5)-\mathrm{C}(21)-\mathrm{C}(22 \mathrm{~A})$ & $110.6(11)$ \\
\hline $\mathrm{C}(12)-\mathrm{C}(13)-\mathrm{Ta}(1)$ & $118.9(6)$ & $\mathrm{N}(5)-\mathrm{C}(21)-\mathrm{C}(23)$ & $108.8(7)$ \\
\hline N(3)-C(14)-H(14) & 128.9 & $\mathrm{~N}(5)-\mathrm{C}(21)-\mathrm{C}(23 \mathrm{~A})$ & $112.0(11)$ \\
\hline $\mathrm{N}(3)-\mathrm{C}(14)-\mathrm{C}(15)$ & $102.2(8)$ & N(5)-C(21)-C(24) & $110.2(8)$ \\
\hline $\mathrm{C}(15)-\mathrm{C}(14)-\mathrm{H}(14)$ & 128.9 & $\mathrm{~N}(5)-\mathrm{C}(21)-\mathrm{C}(24 \mathrm{~A})$ & $104.2(11)$ \\
\hline $\mathrm{N}(4)-\mathrm{C}(15)-\mathrm{C}(14)$ & $107.6(9)$ & $\mathrm{C}(22)-\mathrm{C}(21)-\mathrm{C}(23)$ & $107.8(8)$ \\
\hline N(4)-C(15)-H(15) & 126.2 & $C(22 A)-C(21)-C(24 A)$ & $107.8(9)$ \\
\hline $\mathrm{C}(14)-\mathrm{C}(15)-\mathrm{H}(15)$ & 126.2 & $C(23 A)-C(21)-C(22 A)$ & $112.1(10)$ \\
\hline $\mathrm{N}(3)-\mathrm{C}(16)-\mathrm{Ta}(1)$ & $121.0(7)$ & $C(23 A)-C(21)-C(24 A)$ & $109.7(10)$ \\
\hline $\mathrm{N}(3)-\mathrm{C}(16)-\mathrm{N}(4)$ & $102.5(7)$ & $\mathrm{C}(24)-\mathrm{C}(21)-\mathrm{C}(22)$ & $111.4(8)$ \\
\hline $\mathrm{N}(4)-\mathrm{C}(16)-\mathrm{Ta}(1)$ & $136.4(6)$ & $\mathrm{C}(24)-\mathrm{C}(21)-\mathrm{C}(23)$ & $108.6(8)$ \\
\hline $\mathrm{N}(4)-\mathrm{C}(17)-\mathrm{H}(17 \mathrm{~A})$ & 109.5 & $\mathrm{C}(21)-\mathrm{C}(22)-\mathrm{H}(22 \mathrm{~A})$ & 109.5 \\
\hline $\mathrm{N}(4)-\mathrm{C}(17)-\mathrm{H}(17 \mathrm{~B})$ & 109.5 & $\mathrm{C}(21)-\mathrm{C}(22)-\mathrm{H}(22 \mathrm{~B})$ & 109.5 \\
\hline $\mathrm{N}(4)-\mathrm{C}(17)-\mathrm{C}(18)$ & $110.7(7)$ & $\mathrm{C}(21)-\mathrm{C}(22)-\mathrm{H}(22 \mathrm{C})$ & 109.5 \\
\hline $\mathrm{H}(17 \mathrm{~A})-\mathrm{C}(17)-\mathrm{H}(17 \mathrm{~B})$ & 108.1 & $\mathrm{H}(22 \mathrm{~A})-\mathrm{C}(22)-\mathrm{H}(22 \mathrm{~B})$ & 109.5 \\
\hline C(18)-C(17)-H(17A) & 109.5 & $\mathrm{H}(22 \mathrm{~A})-\mathrm{C}(22)-\mathrm{H}(22 \mathrm{C})$ & 109.5 \\
\hline $\mathrm{C}(18)-\mathrm{C}(17)-\mathrm{H}(17 \mathrm{~B})$ & 109.5 & $\mathrm{H}(22 \mathrm{~B})-\mathrm{C}(22)-\mathrm{H}(22 \mathrm{C})$ & 109.5 \\
\hline $\mathrm{C}(17)-\mathrm{C}(18)-\mathrm{H}(18 \mathrm{~A})$ & 109.5 & $\mathrm{C}(21)-\mathrm{C}(22 \mathrm{~A})-\mathrm{H}(22 \mathrm{D})$ & 109.5 \\
\hline $\mathrm{C}(17)-\mathrm{C}(18)-\mathrm{H}(18 \mathrm{~B})$ & 109.5 & $\mathrm{C}(21)-\mathrm{C}(22 \mathrm{~A})-\mathrm{H}(22 \mathrm{E})$ & 109.5 \\
\hline $\mathrm{C}(17)-\mathrm{C}(18)-\mathrm{C}(19)$ & $110.8(8)$ & $\mathrm{C}(21)-\mathrm{C}(22 \mathrm{~A})-\mathrm{H}(22 \mathrm{~F})$ & 109.5 \\
\hline $\mathrm{H}(18 \mathrm{~A})-\mathrm{C}(18)-\mathrm{H}(18 \mathrm{~B})$ & 108.1 & $\mathrm{H}(22 \mathrm{D})-\mathrm{C}(22 \mathrm{~A})-\mathrm{H}(22 \mathrm{E})$ & 109.5 \\
\hline $\mathrm{C}(19)-\mathrm{C}(18)-\mathrm{H}(18 \mathrm{~A})$ & 109.5 & $\mathrm{H}(22 \mathrm{D})-\mathrm{C}(22 \mathrm{~A})-\mathrm{H}(22 \mathrm{~F})$ & 109.5 \\
\hline $\mathrm{C}(19)-\mathrm{C}(18)-\mathrm{H}(18 \mathrm{~B})$ & 109.5 & $\mathrm{H}(22 \mathrm{E})-\mathrm{C}(22 \mathrm{~A})-\mathrm{H}(22 \mathrm{~F})$ & 109.5 \\
\hline $\mathrm{C}(18)-\mathrm{C}(19)-\mathrm{H}(19 \mathrm{~A})$ & 109.3 & $\mathrm{C}(21)-\mathrm{C}(23)-\mathrm{H}(23 \mathrm{~A})$ & 109.5 \\
\hline $\mathrm{C}(18)-\mathrm{C}(19)-\mathrm{H}(19 \mathrm{~B})$ & 109.3 & $\mathrm{C}(21)-\mathrm{C}(23)-\mathrm{H}(23 \mathrm{~B})$ & 109.5 \\
\hline
\end{tabular}




\begin{tabular}{|c|c|c|c|}
\hline $\mathrm{C}(21)-\mathrm{C}(23)-\mathrm{H}(23 \mathrm{C})$ & 109.5 & $\mathrm{C}(25)-\mathrm{C}(26)-\mathrm{H}(26 \mathrm{~A})$ & 109.5 \\
\hline $\mathrm{H}(23 \mathrm{~A})-\mathrm{C}(23)-\mathrm{H}(23 \mathrm{~B})$ & 109.5 & $\mathrm{C}(25)-\mathrm{C}(26)-\mathrm{H}(26 \mathrm{~B})$ & 109.5 \\
\hline $\mathrm{H}(23 \mathrm{~A})-\mathrm{C}(23)-\mathrm{H}(23 \mathrm{C})$ & 109.5 & $\mathrm{C}(25)-\mathrm{C}(26)-\mathrm{H}(26 \mathrm{C})$ & 109.5 \\
\hline $\mathrm{H}(23 \mathrm{~B})-\mathrm{C}(23)-\mathrm{H}(23 \mathrm{C})$ & 109.5 & $H(26 A)-C(26)-H(26 B)$ & 109.5 \\
\hline $\mathrm{C}(21)-\mathrm{C}(23 \mathrm{~A})-\mathrm{H}(23 \mathrm{D})$ & 109.5 & $\mathrm{H}(26 \mathrm{~A})-\mathrm{C}(26)-\mathrm{H}(26 \mathrm{C})$ & 109.5 \\
\hline $\mathrm{C}(21)-\mathrm{C}(23 \mathrm{~A})-\mathrm{H}(23 \mathrm{E})$ & 109.5 & $\mathrm{H}(26 \mathrm{~B})-\mathrm{C}(26)-\mathrm{H}(26 \mathrm{C})$ & 109.5 \\
\hline $\mathrm{C}(21)-\mathrm{C}(23 \mathrm{~A})-\mathrm{H}(23 \mathrm{~F})$ & 109.5 & $\mathrm{C}(25)-\mathrm{C}(27)-\mathrm{H}(27 \mathrm{~A})$ & 109.5 \\
\hline$H(23 D)-C(23 A)-H(23 E)$ & 109.5 & $\mathrm{C}(25)-\mathrm{C}(27)-\mathrm{H}(27 \mathrm{~B})$ & 109.5 \\
\hline $\mathrm{H}(23 \mathrm{D})-\mathrm{C}(23 \mathrm{~A})-\mathrm{H}(23 \mathrm{~F})$ & 109.5 & $\mathrm{C}(25)-\mathrm{C}(27)-\mathrm{H}(27 \mathrm{C})$ & 109.5 \\
\hline $\mathrm{H}(23 \mathrm{E})-\mathrm{C}(23 \mathrm{~A})-\mathrm{H}(23 \mathrm{~F})$ & 109.5 & $\mathrm{H}(27 \mathrm{~A})-\mathrm{C}(27)-\mathrm{H}(27 \mathrm{~B})$ & 109.5 \\
\hline $\mathrm{C}(21)-\mathrm{C}(24)-\mathrm{H}(24 \mathrm{~A})$ & 109.5 & $\mathrm{H}(27 \mathrm{~A})-\mathrm{C}(27)-\mathrm{H}(27 \mathrm{C})$ & 109.5 \\
\hline $\mathrm{C}(21)-\mathrm{C}(24)-\mathrm{H}(24 \mathrm{~B})$ & 109.5 & $\mathrm{H}(27 \mathrm{~B})-\mathrm{C}(27)-\mathrm{H}(27 \mathrm{C})$ & 109.5 \\
\hline $\mathrm{C}(21)-\mathrm{C}(24)-\mathrm{H}(24 \mathrm{C})$ & 109.5 & $\mathrm{C}(25)-\mathrm{C}(28)-\mathrm{H}(28 \mathrm{~A})$ & 109.5 \\
\hline $\mathrm{H}(24 \mathrm{~A})-\mathrm{C}(24)-\mathrm{H}(24 \mathrm{~B})$ & 109.5 & $\mathrm{C}(25)-\mathrm{C}(28)-\mathrm{H}(28 \mathrm{~B})$ & 109.5 \\
\hline $\mathrm{H}(24 \mathrm{~A})-\mathrm{C}(24)-\mathrm{H}(24 \mathrm{C})$ & 109.5 & $\mathrm{C}(25)-\mathrm{C}(28)-\mathrm{H}(28 \mathrm{C})$ & 109.5 \\
\hline $\mathrm{H}(24 \mathrm{~B})-\mathrm{C}(24)-\mathrm{H}(24 \mathrm{C})$ & 109.5 & $\mathrm{H}(28 \mathrm{~A})-\mathrm{C}(28)-\mathrm{H}(28 \mathrm{~B})$ & 109.5 \\
\hline $\mathrm{C}(21)-\mathrm{C}(24 \mathrm{~A})-\mathrm{H}(24 \mathrm{D})$ & 109.5 & $\mathrm{H}(28 \mathrm{~A})-\mathrm{C}(28)-\mathrm{H}(28 \mathrm{C})$ & 109.5 \\
\hline $\mathrm{C}(21)-\mathrm{C}(24 \mathrm{~A})-\mathrm{H}(24 \mathrm{E})$ & 109.5 & $\mathrm{H}(28 \mathrm{~B})-\mathrm{C}(28)-\mathrm{H}(28 \mathrm{C})$ & 109.5 \\
\hline $\mathrm{C}(21)-\mathrm{C}(24 \mathrm{~A})-\mathrm{H}(24 \mathrm{~F})$ & 109.5 & Ta(1)-Li(1)-Li(1)\#1 & $145.6(6)$ \\
\hline $\mathrm{H}(24 \mathrm{D})-\mathrm{C}(24 \mathrm{~A})-\mathrm{H}(24 \mathrm{E})$ & 109.5 & $\mathrm{I}(1) \# 1-\mathrm{Li}(1)-\mathrm{Ta}(1)$ & $119.9(4)$ \\
\hline $\mathrm{H}(24 \mathrm{D})-\mathrm{C}(24 \mathrm{~A})-\mathrm{H}(24 \mathrm{~F})$ & 109.5 & $\mathrm{I}(1)-\mathrm{Li}(1)-\mathrm{Ta}(1)$ & $119.4(4)$ \\
\hline $\mathrm{H}(24 \mathrm{E})-\mathrm{C}(24 \mathrm{~A})-\mathrm{H}(24 \mathrm{~F})$ & 109.5 & $\mathrm{I}(1)-\mathrm{Li}(1)-\mathrm{I}(1) \# 1$ & $106.3(3)$ \\
\hline $\mathrm{N}(6)-\mathrm{C}(25)-\mathrm{C}(26)$ & $110.1(7)$ & I(1)\#1-Li(1)-Li(1)\#1 & $52.3(3)$ \\
\hline $\mathrm{N}(6)-\mathrm{C}(25)-\mathrm{C}(27)$ & $111.2(5)$ & $\mathrm{I}(1)-\mathrm{Li}(1)-\mathrm{Li}(1) \# 1$ & $53.9(3)$ \\
\hline $\mathrm{N}(6)-\mathrm{C}(25)-\mathrm{C}(28)$ & $108.8(7)$ & $\mathrm{N}(6)-\mathrm{Li}(1)-\mathrm{Ta}(1)$ & $37.0(2)$ \\
\hline $\mathrm{C}(26)-\mathrm{C}(25)-\mathrm{C}(27)$ & 111.2(8) & $\mathrm{N}(6)-\mathrm{Li}(1)-\mathrm{I}(1)$ & $126.8(5)$ \\
\hline$C(26)-C(25)-C(28)$ & 108.1(9) & N(6)-Li(1)-I(1)\#1 & $126.8(5)$ \\
\hline$C(27)-C(25)-C(28)$ & $107.4(7)$ & N(6)-Li(1)-Li(1)\#1 & $77.3(8)$ \\
\hline
\end{tabular}

Symmetry transformations used to generate equivalent atoms: $\# 1-x+2,-y+2,-z+1$ 
Table S8. Torsion Angles for 2.

$\mathrm{Ta}(1)-\mathrm{N}(6)-\mathrm{C}(25)-\mathrm{C}(26)$

$-63.0(13)$

$\mathrm{Ta}(1)-\mathrm{N}(6)-\mathrm{C}(25)-\mathrm{C}(27)$

173.4(7)

$\mathrm{Ta}(1)-\mathrm{N}(6)-\mathrm{C}(25)-\mathrm{C}(28)$

55.3(13)

$\mathrm{N}(1)-\mathrm{C}(5)-\mathrm{C}(6)-\mathrm{N}(2)$

$0.3(9)$

$\mathrm{N}(2)-\mathrm{C}(8)-\mathrm{C}(9)-\mathrm{C}(10)$

177.9(7)

$\mathrm{N}(2)-\mathrm{C}(8)-\mathrm{C}(13)-\mathrm{Ta}(1)$

$\mathrm{N}(2)-\mathrm{C}(8)-\mathrm{C}(13)-\mathrm{C}(12)$

$-176.4(5)$

$\mathrm{N}(3)-\mathrm{C}(12)-\mathrm{C}(13)-\mathrm{Ta}(1)$

$-5.7(8)$

$\mathrm{N}(3)-\mathrm{C}(12)-\mathrm{C}(13)-\mathrm{C}(8)$

176.6(6)

$\mathrm{N}(3)-\mathrm{C}(14)-\mathrm{C}(15)-\mathrm{N}(4)$

$-1.1(10)$

$\mathrm{N}(4)-\mathrm{C}(17)-\mathrm{C}(18)-\mathrm{C}(19)$

$-178.1(8)$

$\mathrm{N}(5)-\mathrm{Ta}(1)-\mathrm{N}(6)-\mathrm{C}(25)$

$-0.4(10)$

$\mathrm{N}(5)-\mathrm{Ta}(1)-\mathrm{N}(6)-\mathrm{Li}(1)$

167.7(4)

$\mathrm{C}(1)-\mathrm{C}(2)-\mathrm{C}(3)-\mathrm{C}(4)$

65.6(13)

$\mathrm{C}(2)-\mathrm{C}(3)-\mathrm{C}(4)-\mathrm{N}(1)$

174.7(9)

$\mathrm{C}(4)-\mathrm{N}(1)-\mathrm{C}(5)-\mathrm{C}(6)$

179.5(7)

$\mathrm{C}(4)-\mathrm{N}(1)-\mathrm{C}(7)-\mathrm{Ta}(1)$

$-0.6(13)$

$\mathrm{C}(4)-\mathrm{N}(1)-\mathrm{C}(7)-\mathrm{N}(2)$

$-179.7(6)$

$\mathrm{C}(5)-\mathrm{N}(1)-\mathrm{C}(4)-\mathrm{C}(3)$

57.1(11)

$\mathrm{C}(5)-\mathrm{N}(1)-\mathrm{C}(7)-\mathrm{Ta}(1)$

179.0(7)

$\mathrm{C}(5)-\mathrm{N}(1)-\mathrm{C}(7)-\mathrm{N}(2)$

$-0.2(9)$

$\mathrm{C}(6)-\mathrm{N}(2)-\mathrm{C}(7)-\mathrm{Ta}(1)$

$-179.1(5)$

$\mathrm{C}(6)-\mathrm{N}(2)-\mathrm{C}(7)-\mathrm{N}(1)$

$0.4(8)$

$\mathrm{C}(6)-\mathrm{N}(2)-\mathrm{C}(8)-\mathrm{C}(9)$

$-3.4(12)$

$\mathrm{C}(6)-\mathrm{N}(2)-\mathrm{C}(8)-\mathrm{C}(13)$

174.3(8)

$\mathrm{C}(7)-\mathrm{Ta}(1)-\mathrm{N}(6)-\mathrm{C}(25)$

$-111.1(10)$

$\mathrm{C}(7)-\mathrm{Ta}(1)-\mathrm{N}(6)-\mathrm{Li}(1)$

$57.1(5)$

$\mathrm{C}(7)-\mathrm{N}(1)-\mathrm{C}(4)-\mathrm{C}(3)$

-123.3(9)

$\mathrm{C}(7)-\mathrm{N}(1)-\mathrm{C}(5)-\mathrm{C}(6)$

$-0.1(10)$

$\mathrm{C}(7)-\mathrm{N}(2)-\mathrm{C}(6)-\mathrm{C}(5)$

$-0.4(9)$

$\mathrm{C}(7)-\mathrm{N}(2)-\mathrm{C}(8)-\mathrm{C}(9)$

179.8(6)

$\mathrm{C}(7)-\mathrm{N}(2)-\mathrm{C}(8)-\mathrm{C}(13)$

$-2.6(8)$

$\mathrm{C}(8)-\mathrm{N}(2)-\mathrm{C}(6)-\mathrm{C}(5)$

$-177.4(7)$

$\mathrm{C}(8)-\mathrm{N}(2)-\mathrm{C}(7)-\mathrm{Ta}(1)$

$-1.6(8)$

$\mathrm{C}(8)-\mathrm{N}(2)-\mathrm{C}(7)-\mathrm{N}(1)$

177.8(6) 


\begin{tabular}{|c|c|}
\hline$C(8)-C(9)-C(10)-C(11)$ & $-2.6(13)$ \\
\hline $\mathrm{C}(9)-\mathrm{C}(8)-\mathrm{C}(13)-\mathrm{Ta}(1)$ & $-176.5(6)$ \\
\hline $\mathrm{C}(9)-\mathrm{C}(8)-\mathrm{C}(13)-\mathrm{C}(12)$ & $1.0(11)$ \\
\hline$C(9)-C(10)-C(11)-C(12)$ & $2.7(13)$ \\
\hline $\mathrm{C}(10)-\mathrm{C}(11)-\mathrm{C}(12)-\mathrm{N}(3)$ & $-178.1(7)$ \\
\hline$C(10)-C(11)-C(12)-C(13)$ & $-0.8(11)$ \\
\hline $\mathrm{C}(11)-\mathrm{C}(12)-\mathrm{C}(13)-\mathrm{Ta}(1)$ & $176.8(6)$ \\
\hline $\mathrm{C}(11)-\mathrm{C}(12)-\mathrm{C}(13)-\mathrm{C}(8)$ & $-0.9(10)$ \\
\hline $\mathrm{C}(12)-\mathrm{N}(3)-\mathrm{C}(14)-\mathrm{C}(15)$ & $178.7(8)$ \\
\hline $\mathrm{C}(12)-\mathrm{N}(3)-\mathrm{C}(16)-\mathrm{Ta}(1)$ & $-1.7(9)$ \\
\hline $\mathrm{C}(12)-\mathrm{N}(3)-\mathrm{C}(16)-\mathrm{N}(4)$ & $-178.7(6)$ \\
\hline $\mathrm{C}(13)-\mathrm{Ta}(1)-\mathrm{N}(6)-\mathrm{C}(25)$ & $177.7(10)$ \\
\hline $\mathrm{C}(13)-\mathrm{Ta}(1)-\mathrm{N}(6)-\mathrm{Li}(1)$ & $-14.1(5)$ \\
\hline $\mathrm{C}(13)-\mathrm{C}(8)-\mathrm{C}(9)-\mathrm{C}(10)$ & $0.7(12)$ \\
\hline $\mathrm{C}(14)-\mathrm{N}(3)-\mathrm{C}(12)-\mathrm{C}(11)$ & $4.9(12)$ \\
\hline $\mathrm{C}(14)-\mathrm{N}(3)-\mathrm{C}(12)-\mathrm{C}(13)$ & $-172.5(7)$ \\
\hline $\mathrm{C}(14)-\mathrm{N}(3)-\mathrm{C}(16)-\mathrm{Ta}(1)$ & $175.8(5)$ \\
\hline $\mathrm{C}(14)-\mathrm{N}(3)-\mathrm{C}(16)-\mathrm{N}(4)$ & $-1.2(9)$ \\
\hline $\mathrm{C}(15)-\mathrm{N}(4)-\mathrm{C}(16)-\mathrm{Ta}(1)$ & $-175.9(7)$ \\
\hline $\mathrm{C}(15)-\mathrm{N}(4)-\mathrm{C}(16)-\mathrm{N}(3)$ & $0.3(9)$ \\
\hline $\mathrm{C}(15)-\mathrm{N}(4)-\mathrm{C}(17)-\mathrm{C}(18)$ & $-62.9(12)$ \\
\hline $\mathrm{C}(16)-\mathrm{Ta}(1)-\mathrm{N}(6)-\mathrm{C}(25)$ & $105.2(10)$ \\
\hline $\mathrm{C}(16)-\mathrm{Ta}(1)-\mathrm{N}(6)-\mathrm{Li}(1)$ & $-86.6(4)$ \\
\hline$C(16)-N(3)-C(12)-C(11)$ & $-177.8(7)$ \\
\hline$C(16)-N(3)-C(12)-C(13)$ & 4.7(9) \\
\hline $\mathrm{C}(16)-\mathrm{N}(3)-\mathrm{C}(14)-\mathrm{C}(15)$ & $1.5(10)$ \\
\hline$C(16)-N(4)-C(15)-C(14)$ & $0.5(11)$ \\
\hline $\mathrm{C}(16)-\mathrm{N}(4)-\mathrm{C}(17)-\mathrm{C}(18)$ & $115.9(8)$ \\
\hline $\mathrm{C}(17)-\mathrm{N}(4)-\mathrm{C}(15)-\mathrm{C}(14)$ & $179.4(8)$ \\
\hline $\mathrm{C}(17)-\mathrm{N}(4)-\mathrm{C}(16)-\mathrm{Ta}(1)$ & $5.1(12)$ \\
\hline $\mathrm{C}(17)-\mathrm{N}(4)-\mathrm{C}(16)-\mathrm{N}(3)$ & $-178.6(7)$ \\
\hline $\mathrm{C}(17)-\mathrm{C}(18)-\mathrm{C}(19)-\mathrm{C}(20)$ & $176.1(10)$ \\
\hline $\mathrm{Li}(1)-\mathrm{Ta}(1)-\mathrm{N}(6)-\mathrm{C}(25)$ & $-168.2(11)$ \\
\hline $\mathrm{Li}(1)-\mathrm{N}(6)-\mathrm{C}(25)-\mathrm{C}(26)$ & $129.2(8)$ \\
\hline $\mathrm{Li}(1)-\mathrm{N}(6)-\mathrm{C}(25)-\mathrm{C}(27)$ & $5.6(8)$ \\
\hline $\mathrm{Li}(1)-\mathrm{N}(6)-\mathrm{C}(25)-\mathrm{C}(28)$ & $-112.5(7)$ \\
\hline
\end{tabular}


Symmetry transformations used to generate equivalent atoms:

\#1 -x+2,-y+2,-z+1

Table S9. Hydrogen Atom Coordinates $\left(\AA \times 10^{4}\right)$ and Isotropic Displacement Parameters $\left(\AA^{2} \times 10^{3}\right)$ for 2 .

\begin{tabular}{|c|c|c|c|c|}
\hline Atom & $\mathrm{X}$ & $\mathrm{y}$ & $\mathrm{z}$ & $\mathrm{U}(\mathrm{eq})$ \\
\hline $\mathrm{H}(1 \mathrm{~A})$ & 10284 & 4331 & 8120 & 197 \\
\hline $\mathrm{H}(1 \mathrm{~B})$ & 11929 & 4629 & 8282 & 197 \\
\hline $\mathrm{H}(1 \mathrm{C})$ & 11023 & 3865 & 7370 & 197 \\
\hline $\mathrm{H}(2 \mathrm{~A})$ & 12690 & 6252 & 7411 & 103 \\
\hline $\mathrm{H}(2 \mathrm{~B})$ & 11652 & 6576 & 8012 & 103 \\
\hline $\mathrm{H}(3 \mathrm{~A})$ & 10721 & 5106 & 6295 & 97 \\
\hline $\mathrm{H}(3 \mathrm{~B})$ & 11178 & 6699 & 6523 & 97 \\
\hline $\mathrm{H}(4 \mathrm{~A})$ & 8612 & 4503 & 7052 & 70 \\
\hline $\mathrm{H}(4 \mathrm{~B})$ & 9099 & 6091 & 7372 & 70 \\
\hline $\mathrm{H}(5)$ & 8650 & 4135 & 5232 & 75 \\
\hline $\mathrm{H}(6)$ & 7176 & 4781 & 4168 & 85 \\
\hline $\mathrm{H}(9)$ & 5855 & 6026 & 3720 & 103 \\
\hline $\mathrm{H}(10)$ & 4551 & 7219 & 3527 & 100 \\
\hline $\mathrm{H}(11)$ & 4139 & 8511 & 4715 & 96 \\
\hline $\mathrm{H}(14)$ & 3765 & 9533 & 6041 & 74 \\
\hline $\mathrm{H}(15)$ & 4249 & 10480 & 7664 & 91 \\
\hline $\mathrm{H}(17 \mathrm{~A})$ & 6810 & 9495 & 8688 & 71 \\
\hline $\mathrm{H}(17 \mathrm{~B})$ & 5387 & 9563 & 8969 & 71 \\
\hline $\mathrm{H}(18 \mathrm{~A})$ & 6432 & 11898 & 8799 & 69 \\
\hline $\mathrm{H}(18 \mathrm{~B})$ & 7843 & 11837 & 8486 & 69 \\
\hline $\mathrm{H}(19 \mathrm{~A})$ & 6930 & 11471 & 10176 & 93 \\
\hline H(19B) & 8288 & 11302 & 9869 & 93 \\
\hline $\mathrm{H}(20 \mathrm{~A})$ & 9452 & 13632 & 9673 & 161 \\
\hline $\mathrm{H}(20 \mathrm{~B})$ & 9092 & 13488 & 10647 & 161 \\
\hline $\mathrm{H}(20 \mathrm{C})$ & 8102 & 13796 & 9996 & 161 \\
\hline
\end{tabular}




\begin{tabular}{|c|c|c|c|}
\hline $\mathrm{H}(22 \mathrm{~A})$ & 4440 & 4430 & 8037 \\
\hline $\mathrm{H}(22 \mathrm{~B})$ & 5166 & 4059 & 8812 \\
\hline $\mathrm{H}(22 \mathrm{C})$ & 5867 & 4267 & 7919 \\
\hline $\mathrm{H}(22 \mathrm{D})$ & 5258 & 4000 & 8237 \\
\hline $\mathrm{H}(22 \mathrm{E})$ & 6546 & 4461 & 8981 \\
\hline $\mathrm{H}(22 \mathrm{~F})$ & 6902 & 4737 & 8020 \\
\hline $\mathrm{H}(23 \mathrm{~A})$ & 8223 & 5977 & 8821 \\
\hline $\mathrm{H}(23 \mathrm{~B})$ & 7353 & 5690 & 9652 \\
\hline $\mathrm{H}(23 \mathrm{C})$ & 8236 & 7225 & 9445 \\
\hline $\mathrm{H}(23 \mathrm{D})$ & 8215 & 7197 & 9397 \\
\hline $\mathrm{H}(23 \mathrm{E})$ & 6914 & 6322 & 9931 \\
\hline $\mathrm{H}(23 \mathrm{~F})$ & 7047 & 7702 & 9630 \\
\hline $\mathrm{H}(24 \mathrm{~A})$ & 6182 & 7522 & 9553 \\
\hline $\mathrm{H}(24 \mathrm{~B})$ & 4966 & 5991 & 9603 \\
\hline $\mathrm{H}(24 \mathrm{C})$ & 4798 & 6789 & 8855 \\
\hline $\mathrm{H}(24 \mathrm{D})$ & 4720 & 6714 & 8904 \\
\hline $\mathrm{H}(24 \mathrm{E})$ & 4271 & 5208 & 9128 \\
\hline $\mathrm{H}(24 \mathrm{~F})$ & 4160 & 5442 & 8148 \\
\hline $\mathrm{H}(26 \mathrm{~A})$ & 9820 & 11379 & 8294 \\
\hline $\mathrm{H}(26 \mathrm{~B})$ & 11209 & 11512 & 8843 \\
\hline $\mathrm{H}(26 \mathrm{C})$ & 9664 & 10213 & 8858 \\
\hline $\mathrm{H}(27 \mathrm{~A})$ & 11941 & 10597 & 6803 \\
\hline $\mathrm{H}(27 \mathrm{~B})$ & 12602 & 11708 & 7652 \\
\hline $\mathrm{H}(27 \mathrm{C})$ & 11355 & 11687 & 7020 \\
\hline $\mathrm{H}(28 \mathrm{~A})$ & 10443 & 8508 & 8386 \\
\hline $\mathrm{H}(28 \mathrm{~B})$ & 12024 & 9798 & 8543 \\
\hline $\mathrm{H}(28 \mathrm{C})$ & 11501 & 8806 & 7641 \\
\hline
\end{tabular}




\section{Experimental}

A suitable crystal was selected and analyzed on a Bruker APEX-II CCD diffractometer. The crystal was kept at $100.0 \mathrm{~K}$ during data collection. Using Olex2, the structure was solved with the ShelXT structure solution program using Direct Methods and refined with the ShelXL refinement package using Least Squares minimization.

\section{Crystal structure determination of 2.}

Crystal Data for $\mathrm{C}_{28} \mathrm{H}_{43} \mathrm{ILiN}_{6} \mathrm{Ta}(M=778.47 \mathrm{~g} / \mathrm{mol})$ : triclinic, space group P-1 (no. 2), $a=$ 10.4680(4) $\AA, \quad b=11.0973(4) \AA, \quad c=15.7314(6) \AA, \quad \alpha=96.431(2)^{\circ}, \quad \beta=92.191(2)^{\circ}, \quad \gamma=$ 117.3560(19) $)^{\circ}, V=1604.53(11) \AA^{3}, Z=2, T=100.0 \mathrm{~K}, \mu(\mathrm{MoK} \alpha)=4.412 \mathrm{~mm}^{-1}$, Dcalc $=$ $1.611 \mathrm{~g} / \mathrm{cm}^{3}, 33007$ reflections measured $\left(4.178^{\circ} \leq 2 \Theta \leq 56.716^{\circ}\right), 7960$ unique $\left(R_{\text {int }}=0.1026\right.$, $\left.\mathrm{R}_{\text {sigma }}=0.0660\right)$ which were used in all calculations. The final $R_{1}$ was $0.0492(\mathrm{I}>2 \sigma(\mathrm{I}))$ and $w R_{2}$ was 0.1250 (all data). 


\section{Computational Results}

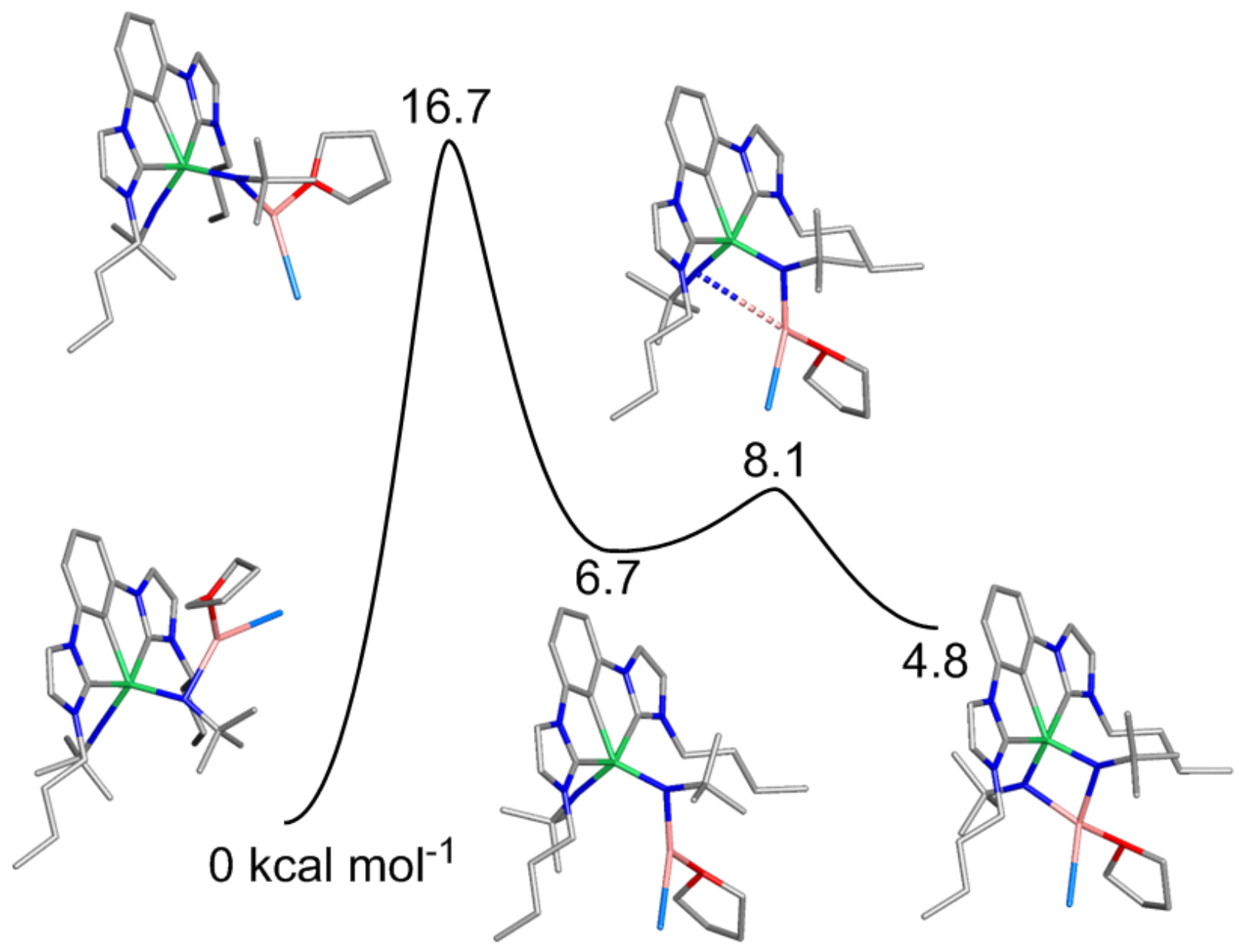

Figure S54. Free energy diagram for the exchange of the lithium iodide between the imido groups in the monomer of $\mathbf{2}$ with an explicitly coordinated THF from PBEPBE-DFT. 


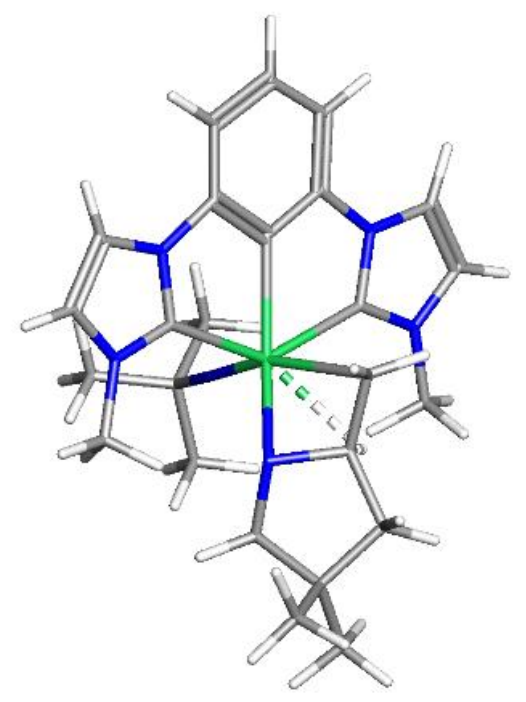

Figure S55. Transition state, TS-8-10, for $\beta$-hydride abstraction in the generation of the oxidative amination product. The computed transition state has a $\Delta \mathrm{G}^{\ddagger}=23.3 \mathrm{kcal} \mathrm{mol}^{-1}$. 


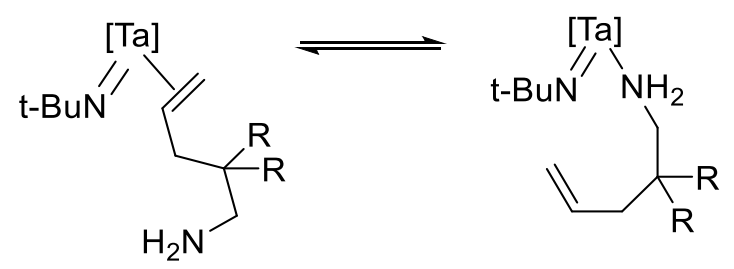

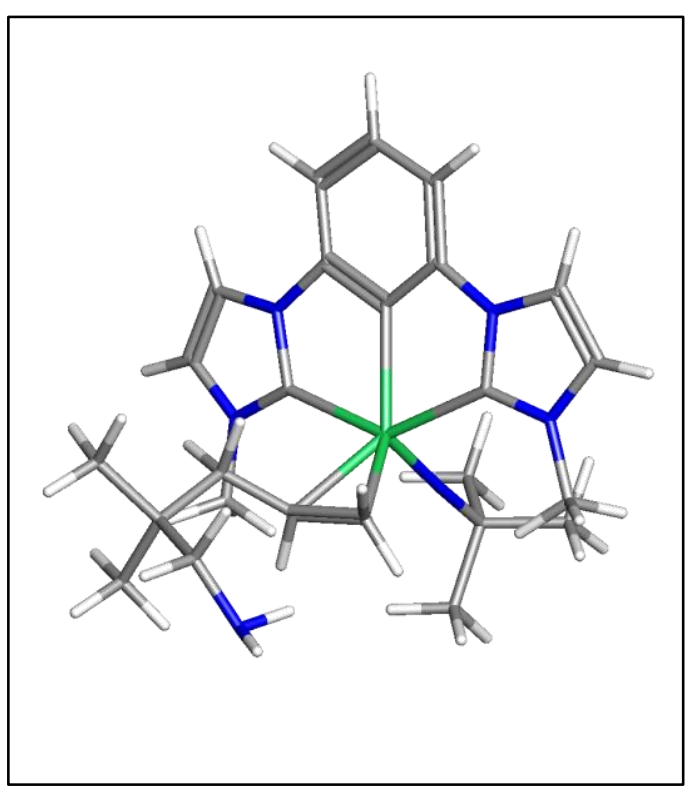

$\Delta G^{\circ}=0.0$ kcal mol$^{-1}$

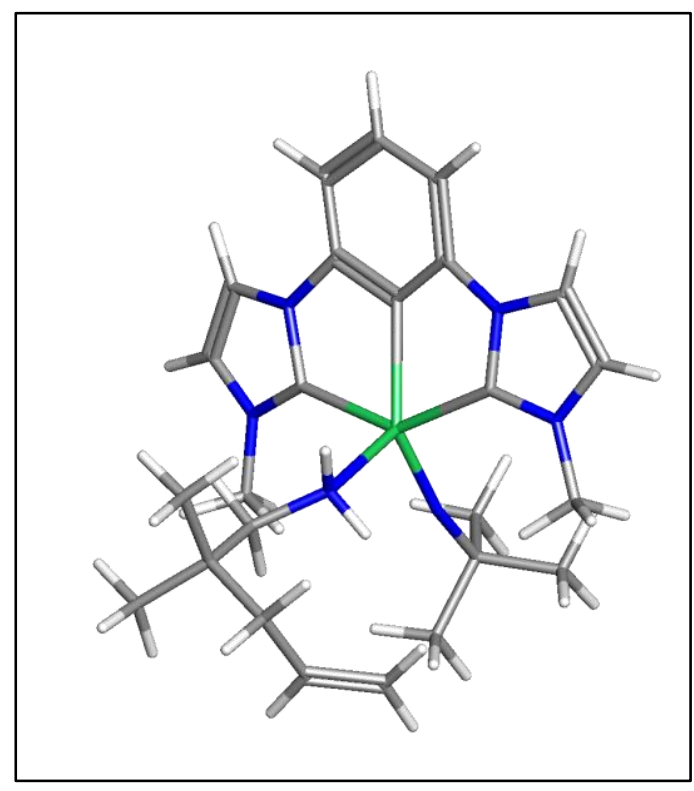

$\Delta \mathrm{G}^{\circ}=23.5 \mathrm{kcal} \mathrm{mol}^{-1}$

Figure S56. Structures and relative energy difference for the Ta(III) olefin versus amine binding. The strong energy difference is suggestive that $\mathrm{Ta}$ (III)-amine is not formed during the catalytic cycle as it is higher than the transition states for the Ta(IV) species.

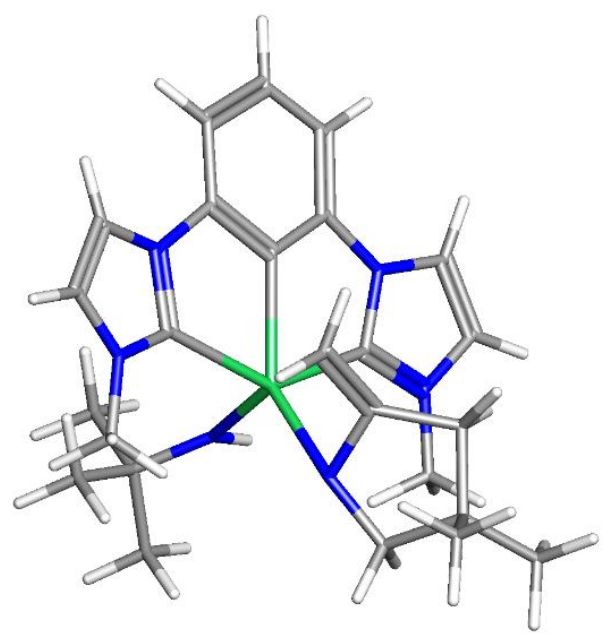

Figure S57. Structure of $\mathbf{1 4}$ with a relative energy of $26.8 \mathrm{kcal} \mathrm{mol}^{-1}$. 


\section{Further Explanation of Computational Details}

The transition state energy from $\mathbf{1 0}$ to $\mathbf{1 3}$ requires some explanation. The addition of a second substrate to 10 affords several different minima, including 10-exchange and 10-exchange'. TS10-13 directly connects 10-exchange' to 13-exchange. However, there are several other structures (including 13-exchange' and 13-exchange") that reflect the same chemical covalent bonding of 13-exchange that have lower energy and can be found be facile rotational transition states. $\mathbf{1 3}$ is the structure of the lowest energy conformer of this imido, hydride complex. In Figure 2, the relative energy of $-5.4 \mathrm{kcal} \mathrm{mol}^{-1}$ at species 13 includes the subsequent rearrangement of $\mathbf{4 i}$ to $\mathbf{4}$.

Preliminary computations using ethylene as a model for the substrate and a $t$-BuNH for the amido ligand are consistent with an accessible transition state for this transformation. Complex model13 (at a relative energy of $5.4 \mathrm{kcal} \mathrm{mol}^{-1}$ compared to infinitely separated species) is the structure used to model complex 13. mTS-13-7 (at $12.9 \mathrm{kcal} \mathrm{mol}^{-1}$ ) is the transition state structure used to model TS-13-7. Complex model-7 (at $-35.9 \mathrm{kcal} \mathrm{mol}^{-1}$ ) is the structure used to model complex 7. The free energy of activation for mTS-13-7 leads to a relatively facile process.

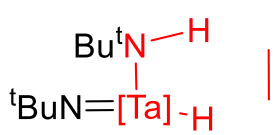

$5.4 \mathrm{kcal} \mathrm{mol}^{-1}$ model-13

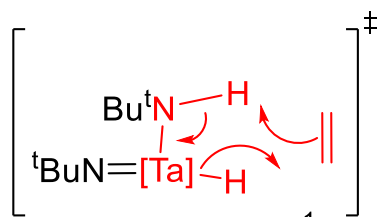

$12.9 \mathrm{kcal} \mathrm{mol}^{-1}$ mTS-13-7

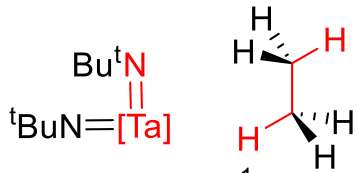

$-35.9 \mathrm{kcal} \mathrm{mol}^{-1}$

model-7 


\section{COMPUTED COORDINATES}

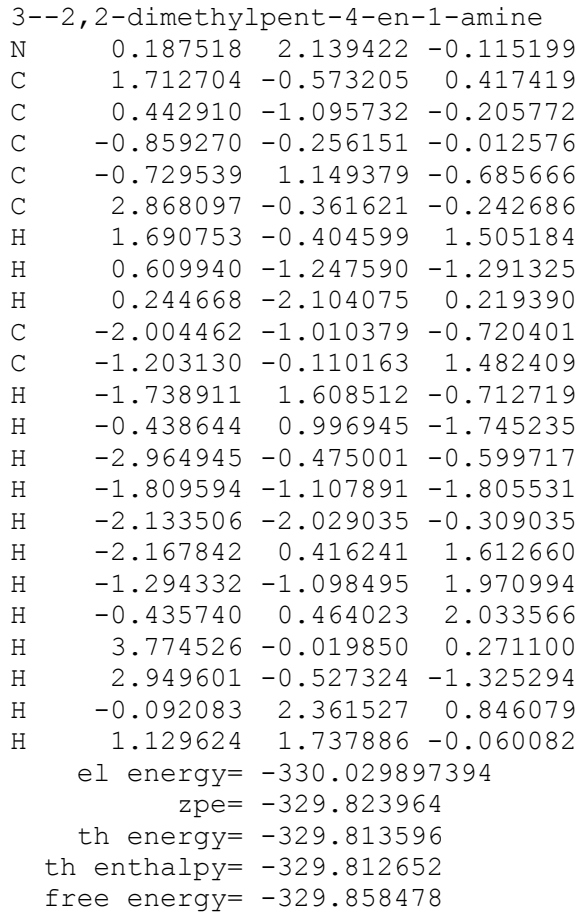

\footnotetext{
4--2, 4, 4-trimethylpyrrolidine

$\mathrm{N} \quad-0.902769-1.216489-0.304740$

$\begin{array}{lllll}\text { C } & -1.392205 & -0.069567 & 0.505572\end{array}$

$\begin{array}{lllll}\text { C } & -0.176695 & 0.896218 & 0.578948\end{array}$

C $\quad 1.030147 \quad 0.110086-0.003962$

C $\quad 0.298114-0.784774-1.031450$

$\begin{array}{lllll}\text { C } & -2.649605 & 0.600782 & -0.070734\end{array}$

$\mathrm{H} \quad-1.641800 \quad-0.445544 \quad 1.520605$

$\mathrm{H} \quad-0.371943 \quad 1.787565 \quad-0.049645$

H $\quad 0.012611 \quad 1.259348 \quad 1.605712$

$\begin{array}{llll}\text { C } & 2.067501 & 1.029471 & -0.664485\end{array}$

C $\quad 1.697925-0.758219 \quad 1.081566$

H $\quad 0.902756-1.662379-1.335709$

$\mathrm{H} \quad 0.089732-0.188731-1.953964$

H $\quad 2.880536 \quad 0.442088-1.131612$

$\mathrm{H} \quad 1.608150 \quad 1.658043-1.450558$

$\begin{array}{llll}\mathrm{H} & 2.533299 & 1.704110 & 0.078233\end{array}$

H $\quad 2.540527-1.337786 \quad 0.659441$

$\mathrm{H} \quad 2.098387-0.131335 \quad 1.900292$

$\mathrm{H} \quad 0.974549-1.474047 \quad 1.509439$

H $\quad-2.986160 \quad 1.438516 \quad 0.568898$

$\mathrm{H} \quad-2.453654 \quad 1.004881 \quad-1.082641$

$\mathrm{H} \quad-1.629425-1.566470-0.935242$

$\mathrm{H} \quad-\quad-3.489284-0.116814 \quad-0.142795$

el energy $=-330.061840318$ $\mathrm{zpe}=-329.853506$

th energy $=-329.844319$

th enthalpy $=-329.843375$

free energy $=-329.886971$
}

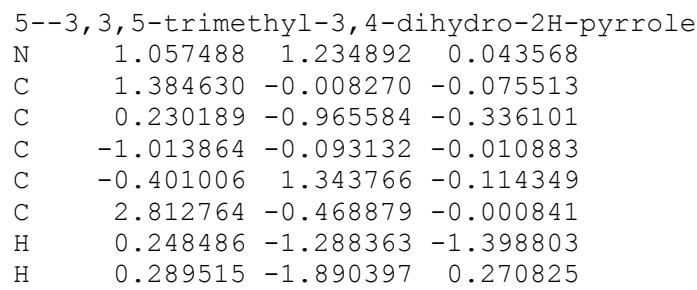

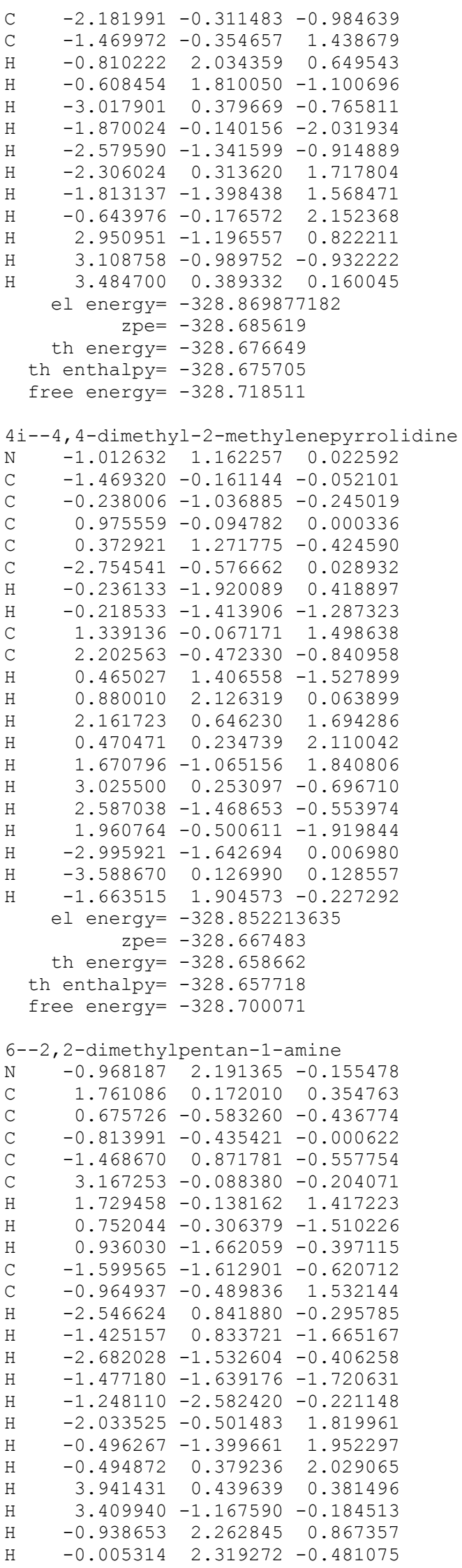




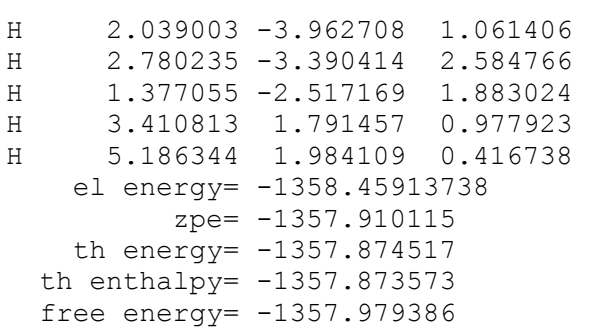

\begin{tabular}{|c|c|c|c|}
\hline \multicolumn{4}{|c|}{ TS $-7-8$} \\
\hline & -0.176397 & 0.126901 & 0.214601 \\
\hline $\mathrm{N}$ & 1.070456 & -2.581442 & -0.931585 \\
\hline I & -0.733482 & -3.225208 & 0.080412 \\
\hline $\mathrm{N}$ & 0.812250 & 3.359745 & 0.040504 \\
\hline C & 2.610539 & 0.677084 & -1.361035 \\
\hline $\mathrm{N}$ & 2.166236 & 1.967789 & -0.922398 \\
\hline C & -0.027654 & -2.099453 & -0.243418 \\
\hline C & 1.728050 & -0.333518 & -0.962419 \\
\hline C & 2.049643 & -1.632292 & -1.370824 \\
\hline C & -1.943463 & -3.208271 & 0.893810 \\
\hline $\mathrm{H}$ & -1.735687 & -3.618910 & 1.898126 \\
\hline $\mathrm{H}$ & -2.262957 & -2.157590 & 0.991520 \\
\hline C & 0.964292 & 2.031665 & -0.243929 \\
\hline C & 3.193510 & -1.946754 & -2.122983 \\
\hline $\mathrm{H}$ & 3.431795 & -2.972005 & -2.432505 \\
\hline C & -0.100632 & -4.373827 & -0.386090 \\
\hline $\mathrm{H}$ & -0.509403 & -5.369883 & -0.224147 \\
\hline C & 2.742087 & 3.223279 & -1.047954 \\
\hline $\mathrm{H}$ & 3.692630 & 3.389143 & -1.550270 \\
\hline $\mathrm{H}$ & -0.766625 & 4.743513 & 0.258678 \\
\hline C & 1.886435 & 4.105121 & -0.436497 \\
\hline $\mathrm{H}$ & 1.950707 & 5.183948 & -0.304148 \\
\hline $\mathrm{H}$ & -2.737745 & -3.802096 & 0.408674 \\
\hline C & 1.040067 & -3.964846 & -1.029044 \\
\hline $\mathrm{H}$ & 1.814683 & -4.537980 & -1.533759 \\
\hline C & -0.314905 & 3.893107 & 0.798627 \\
\hline $\mathrm{H}$ & -1.050105 & 3.078367 & 0.914155 \\
\hline $\mathrm{H}$ & 0.018199 & 4.226725 & 1.797604 \\
\hline $\mathrm{C}$ & 3.772985 & 0.439303 & -2.111629 \\
\hline $\mathrm{H}$ & 4.456163 & 1.243772 & -2.411411 \\
\hline C & 4.052326 & -0.890357 & -2.481611 \\
\hline $\mathrm{H}$ & 4.953702 & -1.106422 & -3.065989 \\
\hline $\mathrm{N}$ & 0.568744 & -0.073771 & 1.886220 \\
\hline $\mathrm{C}$ & 1.328493 & -0.281332 & 3.082544 \\
\hline $\mathrm{C}$ & 2.848140 & -0.302113 & 2.776330 \\
\hline $\mathrm{H}$ & 3.079925 & -1.106812 & 2.056205 \\
\hline $\mathrm{H}$ & 3.153907 & 0.656251 & 2.318688 \\
\hline $\mathrm{H}$ & 3.450991 & -0.460238 & 3.692303 \\
\hline C & 0.908661 & -1.638424 & 3.703941 \\
\hline $\mathrm{H}$ & 1.113154 & -2.459973 & 2.993161 \\
\hline $\mathrm{H}$ & 1.462573 & -1.840485 & 4.640877 \\
\hline $\mathrm{H}$ & -0.173388 & -1.634382 & 3.928001 \\
\hline $\mathrm{C}$ & 1.019548 & 0.857458 & 4.089409 \\
\hline $\mathrm{H}$ & -0.060126 & 0.879028 & 4.321510 \\
\hline $\mathrm{H}$ & 1.584156 & 0.730476 & 5.033444 \\
\hline $\mathrm{H}$ & 1.294555 & 1.833798 & 3.649490 \\
\hline $\mathrm{N}$ & -2.013209 & 0.661973 & 0.450161 \\
\hline $\mathrm{C}$ & -1.965432 & 0.773057 & -1.727554 \\
\hline C & -3.278358 & 0.022931 & -1.750883 \\
\hline C & -4.259418 & 0.488139 & -0.641980 \\
\hline C & -3.364743 & 1.092909 & 0.510083 \\
\hline C & -0.786919 & 0.267065 & -2.293803 \\
\hline $\mathrm{H}$ & -2.057812 & 1.869874 & -1.679881 \\
\hline $\mathrm{H}$ & -3.748424 & 0.160466 & -2.747792 \\
\hline $\mathrm{H}$ & -3.059486 & -1.058272 & -1.656674 \\
\hline C & -5.224083 & 1.568008 & -1.171674 \\
\hline C & -5.076822 & -0.707634 & -0.118721 \\
\hline $\mathrm{H}$ & -3.823774 & 0.845215 & 1.496094 \\
\hline $\mathrm{H}$ & -3.414479 & 2.207458 & 0.434645 \\
\hline
\end{tabular}




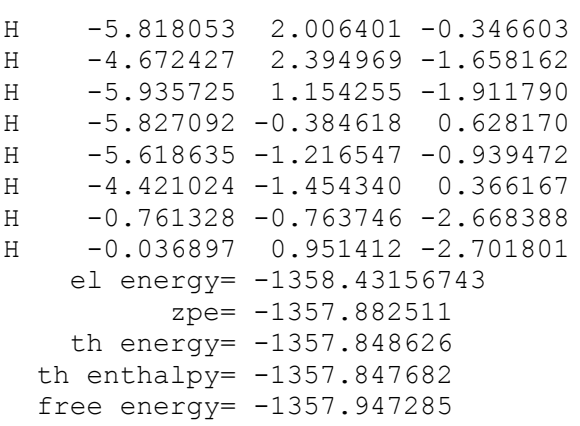

$\begin{array}{lllll}\mathrm{Ta} & 0.043144 & -0.101849 & 0.166443\end{array}$

$\mathrm{N} \quad-1.344322 \quad 2.609976-0.752402$

$\begin{array}{llll}0.586990 & 3.243763-0.002901\end{array}$

$-0.882293-3.346038-0.014757$

$-2.875198-0.661422-1.088208$

$-2.372857-1.953237-0.747974$

$\begin{array}{lll}-0.174794 & 2.124606 & -0.203224\end{array}$

$\begin{array}{llll}-1.971870 & 0.353396 & -0.733393\end{array}$

$\begin{array}{lll}-2.350402 & 1.656772 & -1.091956\end{array}$

$\begin{array}{lll}1.903343 & 3.222562 & 0.620762\end{array}$

$\begin{array}{lll}1.835671 & 3.516407 & 1.683830\end{array}$

$\begin{array}{llll}2.294411 & 2.194460 & 0.554978\end{array}$

$-1.106937-2.009098-0.201835$

$\begin{array}{lll}-3.559414 & 1.966616 & -1.733615\end{array}$

$\begin{array}{lll}-3.832268 & 2.995072 & -2.000383\end{array}$

$\begin{array}{lll}-0.081984 & 4.391586 & -0.419043\end{array}$

$0.362381 \quad 5.382693-0.343933$

$-2.914891-3.220883-0.899690$

$-3.901738-3.394696-1.322705$

$\begin{array}{lll}1.144122 & -3.153418 & 0.420019\end{array}$

$-1.972977-4.102509-0.431991$

$-1.985811-5.189203-0.365685$

$\begin{array}{llll}2.582279 & 3.911007 & 0.088807\end{array}$

$\begin{array}{llll}-1.303283 & 3.989307 & -0.896290\end{array}$

$\begin{array}{llll}-2.123985 & 4.563517 & -1.320227\end{array}$

$\begin{array}{llll}0.332730 & -3.880462 & 0.588556\end{array}$

$\begin{array}{lll}0.197974 & -4.023782 & 1.676092\end{array}$

$\begin{array}{lll}0.586895 & -4.844913 & 0.117850\end{array}$

$-4.100403-0.423787-1.729089$

$-4.789353-1.236085-1.991468$

$\begin{array}{lll}-4.434386 & 0.907672 & -2.039851\end{array}$

$\begin{array}{llll}-5.386903 & 1.122248 & -2.536854\end{array}$

$\begin{array}{lll}-0.127440 & -0.004145 & 2.001067\end{array}$

$\begin{array}{lll}-0.419082 & 0.133712 & 3.403929\end{array}$

$\begin{array}{lll}-1.946823 & 0.022754 & 3.628261\end{array}$

$\begin{array}{lll}-2.471636 & 0.814499 & 3.064698\end{array}$

$\begin{array}{lll}-2.312019 & -0.952132 & 3.257984\end{array}$

$\begin{array}{lll}-2.209919 & 0.114497 & 4.699846\end{array}$

$\begin{array}{lll}0.081228 & 1.514389 & 3.897306\end{array}$

$\begin{array}{lll}-0.414141 & 2.322411 & 3.328804\end{array}$

$\begin{array}{lll}-0.134218 & 1.659843 & 4.973102\end{array}$

$\begin{array}{lll}1.172933 & 1.602015 & 3.746193\end{array}$

$0.306429-0.988520 \quad 4.187157$

$1.397688-0.925105 \quad 4.025645$

$0.106299-0.918929 \quad 5.273562$

$\begin{array}{lll}-0.034742 & -1.978450 & 3.832498\end{array}$

$1.946196-0.667412-0.286361$

$1.918277-0.768456-1.769293$

$3.218752-0.041737-2.169286$

$4.246856-0.435646-1.062975$

$3.320701-0.894524 \quad 0.134840$

$0.555797-0.210926-2.184492$

$2.014733-1.845638-2.056470$

$3.570381-0.303905-3.184811$

$\begin{array}{llll}3.029674 & 1.050254 & -2.148444\end{array}$

$5.134482-1.605949-1.532190$

$$
\begin{array}{rrr}
5.135875 & 0.752293 & -0.655104 \\
3.541524 & -0.356831 & 1.077020 \\
3.495629 & -1.977098 & 0.337530 \\
5.768247 & -1.986296 & -0.707920 \\
4.520337 & -2.450085 & -1.898754 \\
5.805129 & -1.295027 & -2.355576 \\
5.856555 & 0.467589 & 0.135779 \\
5.718024 & 1.130110 & -1.517185 \\
4.527191 & 1.592127 & -0.270019 \\
-0.019695 & -0.880775 & -2.845028 \\
0.642751 & 0.777774 & -2.671538 \\
\text { el energy }= & -1358.46233264 \\
\text { zpe }= & -1357.911337 \\
\text { th energy }= & -1357.877326 \\
\text { th enthalpy }= & -1357.876382 \\
\text { free energy }= & -1357.977292
\end{array}
$$

\begin{tabular}{|c|c|c|c|}
\hline \multicolumn{4}{|c|}{$\mathrm{TS}-8-10$} \\
\hline $\mathrm{Ta}$ & 0.055968 & 0.026230 & -0.037229 \\
\hline & -1.809916 & 2.619130 & -0.233550 \\
\hline N & 0.123596 & 3.409741 & 0.317161 \\
\hline N & -0.603263 & -3.327205 & -0.387569 \\
\hline $\mathrm{C}$ & -2.944099 & -0.760125 & -0.883637 \\
\hline & -2.267670 & -2.009867 & -0.803302 \\
\hline C & -0.519310 & 2.233644 & 0.055905 \\
\hline C & -2.114893 & 0.322721 & -0.552600 \\
\hline C & -2.725398 & 1.584868 & -0.588752 \\
\hline C & 1.544370 & 3.501992 & 0.63 \\
\hline H & 1.733191 & 3.072961 & 1.637551 \\
\hline & 2.098916 & 2.917709 & -0.1 \\
\hline $\mathrm{C}$ & -0.944342 & -1.999607 & -0.411806 \\
\hline C & -4.066793 & 1.796573 & -0.9 \\
\hline $\mathrm{H}$ & -4.512979 & 2.798079 & -0.957194 \\
\hline $\mathrm{C}$ & -0.738258 & 4.493079 & 0.1 \\
\hline $\mathrm{H}$ & -0.407781 & 5.516373 & 0.370009 \\
\hline $\mathrm{C}$ & -2.727085 & -3.299280 & -1.033053 \\
\hline $\mathrm{H}$ & -3.745852 & -3.517842 & -1.344131 \\
\hline $\mathrm{H}$ & 1.376207 & -3.929726 & -0.86 \\
\hline $\mathrm{C}$ & -1.673032 & -4.133392 & -0.773373 \\
\hline $\mathrm{H}$ & -1.592320 & -5.217880 & -0.8 \\
\hline $\mathrm{H}$ & 1.846673 & 4.561199 & 0.6231 \\
\hline $\mathrm{C}$ & -1.967286 & 3.992313 & -0.1 \\
\hline $\mathrm{H}$ & -2.911419 & 4.494921 & -0.34788 \\
\hline $\mathrm{C}$ & 0.701977 & -3.847417 & 0.0 \\
\hline $\mathrm{H}$ & 1.149814 & -3.164898 & 0.7429 \\
\hline $\mathrm{H}$ & 0.571146 & -4.843454 & 0.4 \\
\hline $\mathrm{C}$ & -4.294815 & -0.620623 & -1.2441 \\
\hline $\mathrm{H}$ & -4.920155 & -1.484492 & $-1 \cdot 4$ \\
\hline $\mathrm{C}$ & -4.845034 & 0.672052 & -1.267508 \\
\hline $\mathrm{H}$ & -5.896561 & 0.806043 & -1.544409 \\
\hline $\mathrm{N}$ & 0.118502 & -0.265638 & 1.781381 \\
\hline $\mathrm{C}$ & 0.072051 & -0.702569 & 3.15 \\
\hline $\mathrm{C}$ & 1.410296 & -1.377759 & 3.540361 \\
\hline $\mathrm{H}$ & 1.586017 & -2.274830 & 2.91793 \\
\hline $\mathrm{H}$ & 2.254084 & -0.683091 & $3.3^{\circ}$ \\
\hline $\mathrm{H}$ & 1.410700 & -1.691877 & \\
\hline C & -1.096762 & -1.698745 & 3.34 \\
\hline $\mathrm{H}$ & -0.949180 & -2.595370 & \\
\hline $\mathrm{H}$ & -1.179034 & -2.022739 & 5097 \\
\hline $\mathrm{H}$ & -2.048570 & -1.226674 & \\
\hline $\mathrm{C}$ & -0.154891 & 0.539477 & 4.052852 \\
\hline $\mathrm{H}$ & -1.099829 & 1.040240 & 3.776577 \\
\hline $\mathrm{H}$ & -0.204512 & 0.256499 & 5.121631 \\
\hline $\mathrm{H}$ & 0.669493 & 1.263591 & 3.920748 \\
\hline $\mathrm{N}$ & 2.000608 & 0.692015 & -0.645496 \\
\hline $\mathrm{C}$ & 1.882535 & -0.503859 & -1.506592 \\
\hline $\mathrm{C}$ & 3.318857 & -0.705670 & -2.078522 \\
\hline $\mathrm{C}$ & 4.267752 & -0.025653 & -1.032547 \\
\hline $\mathrm{C}$ & 3.260378 & 0.464268 & 0.064931 \\
\hline $\mathrm{C}$ & 0.599299 & -0.342526 & -2.29154 \\
\hline $\mathrm{H}$ & 1.733110 & -1.446732 & -0.83031 \\
\hline
\end{tabular}




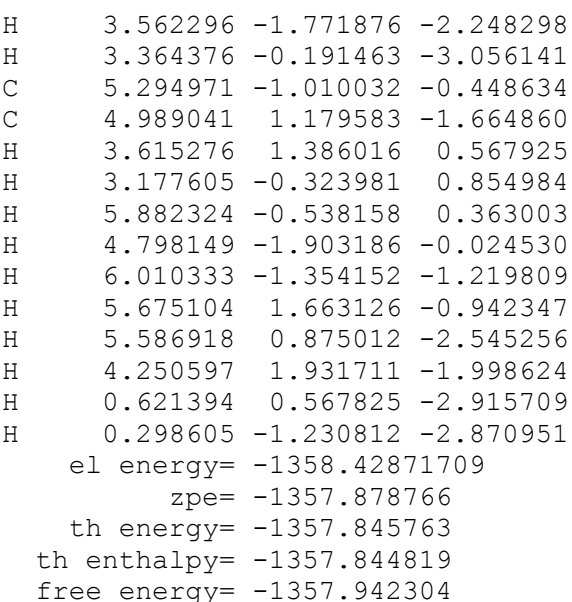

$\begin{array}{lrrr}\text { I0 } & & & \\ \mathrm{Ta} & 0.114937 & -0.150256 & 0.108258 \\ \mathrm{~N} & -1.341724 & 2.641670 & -0.632285 \\ \mathrm{~N} & 0.500305 & 3.321460 & 0.260646 \\ \mathrm{~N} & -0.996769 & -3.394309 & -0.056835 \\ \mathrm{C} & -2.781203 & -0.634763 & -1.270372 \\ \mathrm{~N} & -2.325757 & -1.945204 & -0.950841 \\ \mathrm{C} & -0.160785 & 2.177301 & -0.088895 \\ \mathrm{C} & -1.872318 & 0.352924 & -0.859927 \\ \mathrm{C} & -2.285591 & 1.675709 & -1.089913 \\ \mathrm{C} & 1.808211 & 3.342732 & 0.908152 \\ \mathrm{H} & 1.697958 & 3.208177 & 1.998906 \\ \mathrm{H} & 2.393388 & 2.509128 & 0.485622 \\ \mathrm{C} & -1.107536 & -2.047487 & -0.304783 \\ \mathrm{C} & -3.505589 & 2.018404 & -1.698185 \\ \mathrm{H} & -3.798247 & 3.062324 & -1.861356 \\ \mathrm{C} & -0.243457 & 4.457380 & -0.044133 \\ \mathrm{H} & 0.120753 & 5.461944 & 0.165162 \\ \mathrm{C} & -2.942232 & -3.175041 & -1.108022 \\ \mathrm{H} & -3.904443 & -3.300496 & -1.599079 \\ \mathrm{H} & 0.941466 & -3.345722 & 0.676846 \\ \mathrm{C} & -2.100006 & -4.092489 & -0.536522 \\ \mathrm{H} & -2.189937 & -5.172207 & -0.429502 \\ \mathrm{H} & 2.297328 & 4.309229 & 0.702603 \\ \mathrm{C} & -1.413079 & 4.027661 & -0.614790 \\ \mathrm{H} & -2.263062 & 4.585765 & -1.000262 \\ \mathrm{C} & 0.080590 & -4.035640 & 0.689493 \\ \mathrm{H} & -0.231595 & -4.214529 & 1.734355 \\ \mathrm{H} & 0.336166 & -4.996738 & 0.211344 \\ \mathrm{C} & -4.011346 & -0.363960 & -1.888770 \\ \mathrm{H} & -4.693770 & -1.163486 & -2.200489 \\ \mathrm{C} & -4.361795 & 0.980637 & -2.100155 \\ \mathrm{H} & -5.316982 & 1.222039 & -2.579555 \\ \mathrm{~N} & -0.249554 & -0.094793 & 1.887232 \\ \mathrm{C} & -0.629108 & -0.059571 & 3.272830 \\ \mathrm{C} & -0.456858 & 1.380091 & 3.817428 \\ \mathrm{H} & 0.600716 & 1.692422 & 3.741186 \\ \mathrm{H} & -1.071170 & 2.086774 & 3.231197 \\ \mathrm{H} & -0.762774 & 1.444749 & 4.879075 \\ \mathrm{C} & 0.269336 & -1.030529 & 4.078000 \\ \mathrm{H} & 1.330286 & -0.735903 & 3.989139 \\ \mathrm{H} & -0.006597 & -1.036626 & 5.149846 \\ \mathrm{H} & 0.168359 & -2.057705 & 3.683653 \\ \mathrm{C} & -2.112053 & -0.488485 & 3.403566 \\ \mathrm{H} & -2.247887 & -1.510228 & 3.005843 \\ \mathrm{H} & -2.445718 & -0.473547 & 4.458843 \\ \mathrm{H} & -2.755819 & 0.190814 & 2.816968 \\ \mathrm{~N} & 2.242523 & 0.504219 & -0.396232 \\ \mathrm{C} & 2.094582 & -0.067076 & -1.626798 \\ \mathrm{C} & 3.218521 & -1.046667 & -1.927818 \\ \mathrm{C} & 4.336032 & -0.596779 & -0.949144\end{array}$

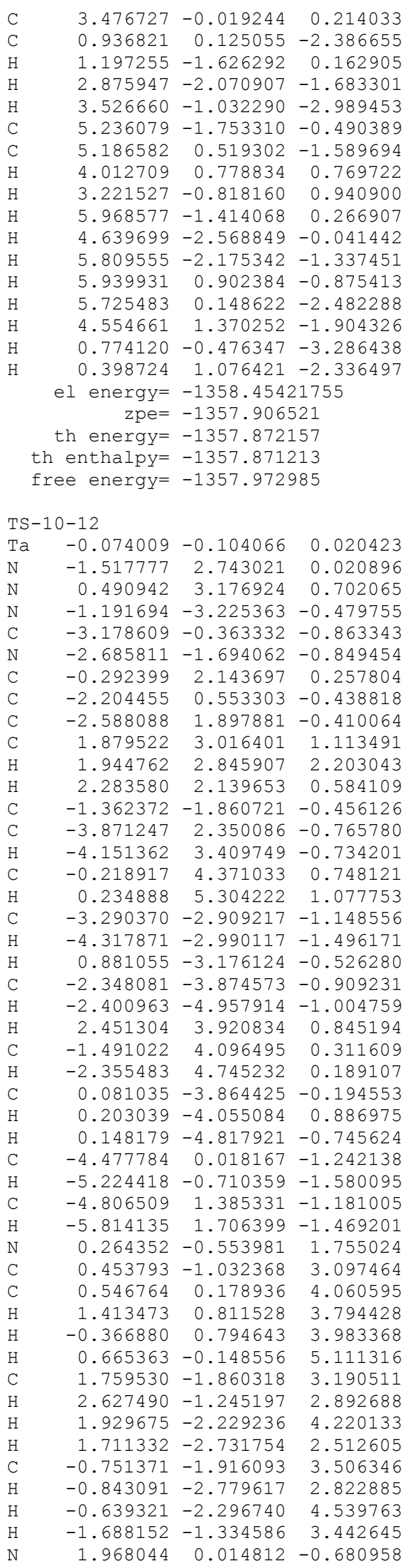




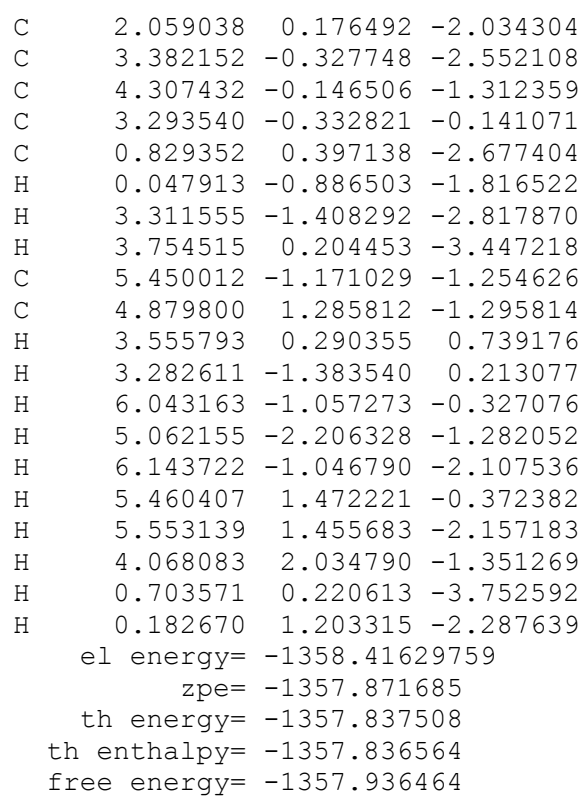

\begin{tabular}{|c|c|c|c|}
\hline $\mathrm{Ta}$ & 0.057134 & -0.062954 & 0.179548 \\
\hline & 2.349710 & -2.095996 & -0.589014 \\
\hline N & 0.716488 & -3.383245 & 0.047689 \\
\hline $\mathrm{N}$ & -0.079595 & 3.279250 & 0.532407 \\
\hline C & 2.739946 & 1.517501 & -0.609471 \\
\hline N & 1.807517 & 2.525909 & -0.260067 \\
\hline C & 1.012012 & -2.052266 & -0.181992 \\
\hline $\mathrm{C}$ & 2.222335 & 0.231830 & -0.389243 \\
\hline C & 3.017410 & -0.852162 & -0.782194 \\
\hline C & -0.577188 & -3.852150 & 0.514902 \\
\hline $\mathrm{H}$ & -0.533901 & -4.134379 & 1.582370 \\
\hline $\mathrm{H}$ & -1.292283 & -3.020755 & 0.401988 \\
\hline C & 0.516269 & 2.103371 & 0.100525 \\
\hline C & 4.308098 & -0.700602 & -1.327318 \\
\hline $\mathrm{H}$ & 4.918137 & -1.555848 & -1.641030 \\
\hline C & 1.805880 & -4.204359 & -0.218143 \\
\hline $\mathrm{H}$ & 1.759826 & -5.286009 & -0.100075 \\
\hline C & 1.970693 & 3.892466 & -0.080759 \\
\hline $\mathrm{H}$ & 2.897196 & 4.412700 & -0.314383 \\
\hline $\mathrm{H}$ & -2.032057 & 2.564230 & 0.526925 \\
\hline $\mathrm{C}$ & 0.787571 & 4.361692 & 0.428928 \\
\hline $\mathrm{H}$ & 0.491544 & 5.370083 & 0.714784 \\
\hline $\mathrm{H}$ & -0.904685 & -4.722331 & -0.082004 \\
\hline C & 2.833825 & -3.394099 & -0.629240 \\
\hline $\mathrm{H}$ & 3.851614 & -3.636531 & -0.927558 \\
\hline $\mathrm{C}$ & -1.441366 & 3.350335 & 1.029161 \\
\hline $\mathrm{H}$ & -1.483004 & 3.170808 & 2.119505 \\
\hline $\mathrm{H}$ & -1.867342 & 4.344118 & 0.806142 \\
\hline $\mathrm{C}$ & 4.026238 & 1.739445 & -1.145998 \\
\hline $\mathrm{H}$ & 4. 421884 & 2.747116 & -1.320284 \\
\hline $\mathrm{C}$ & 4.795667 & 0.611755 & -1.483235 \\
\hline $\mathrm{H}$ & 5.797624 & 0.758999 & -1.903952 \\
\hline N & -0.614007 & -0.265566 & 1.859015 \\
\hline $\mathrm{C}$ & -0.846656 & -0.325080 & 3.280843 \\
\hline $\mathrm{C}$ & -2.335481 & -0.014708 & 3.576885 \\
\hline $\mathrm{H}$ & -2.596186 & 0.997375 & 3.217152 \\
\hline $\mathrm{H}$ & -2.988705 & -0.740033 & 3.058468 \\
\hline $\mathrm{H}$ & -2.551451 & -0.064352 & 4.661460 \\
\hline $\mathrm{C}$ & 0.051090 & 0.709050 & 4.004790 \\
\hline $\mathrm{H}$ & -0.163190 & 1.727787 & 3.633869 \\
\hline $\mathrm{H}$ & -0.112949 & 0.693494 & 5.099813 \\
\hline $\mathrm{H}$ & 1.115788 & 0.494963 & 3.802466 \\
\hline $\mathrm{C}$ & -0.509728 & -1.743869 & 3.803764 \\
\hline $\mathrm{H}$ & 0.539535 & -1.996559 & 3.568262 \\
\hline
\end{tabular}

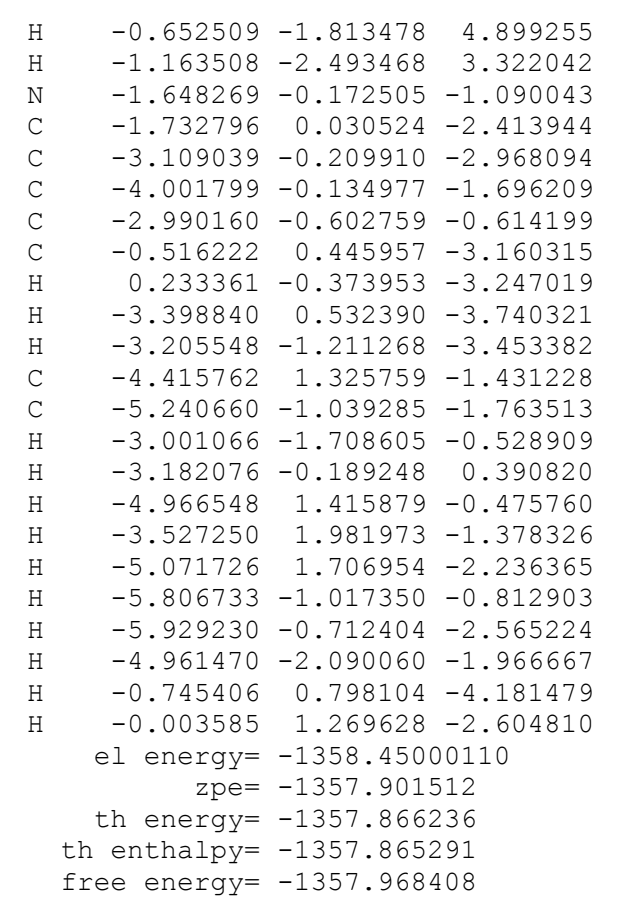

$\begin{array}{lrrr}12^{\prime} & & & \\ \mathrm{Ta} & 0.048888 & 0.075038 & -0.030871 \\ \mathrm{~N} & -2.610155 & 1.808170 & -0.212037 \\ \mathrm{~N} & -1.096049 & 3.248213 & 0.345089 \\ \mathrm{~N} & 0.581134 & -3.359092 & -0.007704 \\ \mathrm{C} & -2.504673 & -1.799066 & -0.621457 \\ \mathrm{~N} & -1.429764 & -2.731598 & -0.472474 \\ \mathrm{C} & -1.255679 & 1.921212 & 0.052354 \\ \mathrm{C} & -2.106432 & -0.477384 & -0.384861 \\ \mathrm{C} & -3.107803 & 0.499863 & -0.488590 \\ \mathrm{C} & 0.182234 & 3.835889 & 0.722704 \\ \mathrm{H} & 0.266938 & 3.904875 & 1.821915 \\ \mathrm{H} & 0.972562 & 3.170528 & 0.337801 \\ \mathrm{C} & -0.178953 & -2.234013 & -0.162353 \\ \mathrm{C} & -4.442364 & 0.206834 & -0.811824 \\ \mathrm{H} & -5.209301 & 0.986526 & -0.890308 \\ \mathrm{C} & -2.302208 & 3.937582 & 0.266602 \\ \mathrm{H} & -2.374885 & 5.005089 & 0.468027 \\ \mathrm{C} & -1.440041 & -4.117751 & -0.515128 \\ \mathrm{H} & -2.332983 & -4.698843 & -0.734256 \\ \mathrm{H} & 2.253315 & -2.331583 & 0.676777 \\ \mathrm{C} & -0.162892 & -4.517183 & -0.220145 \\ \mathrm{H} & 0.272532 & -5.511879 & -0.139455 \\ \mathrm{H} & 0.274746 & 4.842477 & 0.280510 \\ \mathrm{C} & -3.262405 & 3.026349 & -0.092609 \\ \mathrm{H} & -4.328949 & 3.149758 & -0.267316 \\ \mathrm{C} & 1.990269 & -3.352917 & 0.365121 \\ \mathrm{H} & 2.156706 & -4.049514 & 1.205259 \\ \mathrm{H} & 2.621352 & -3.653397 & -0.490029 \\ \mathrm{C} & -3.821219 & -2.162174 & -0.949377 \\ \mathrm{H} & -4.112569 & -3.202740 & -1.134488 \\ \mathrm{C} & -4.781604 & -1.138645 & -1.041184 \\ \mathrm{H} & -5.815810 & -1.395160 & -1.297117 \\ \mathrm{~N} & 0.598309 & 0.076536 & 1.717582 \\ \mathrm{C} & 0.918427 & -0.006794 & 3.119135 \\ \mathrm{C} & 2.444607 & 0.108295 & 3.354262 \\ \mathrm{H} & 2.978565 & -0.734086 & 2.878393 \\ \mathrm{H} & 2.832552 & 1.046256 & 2.918210 \\ \mathrm{H} & 2.684924 & 0.099072 & 4.434741 \\ \mathrm{C} & 0.413751 & -1.359923 & 3.681240 \\ \mathrm{H} & 0.901632 & -2.199146 & 3.152931 \\ \mathrm{H} & 0.633075 & -1.454645 & 4.762160\end{array}$




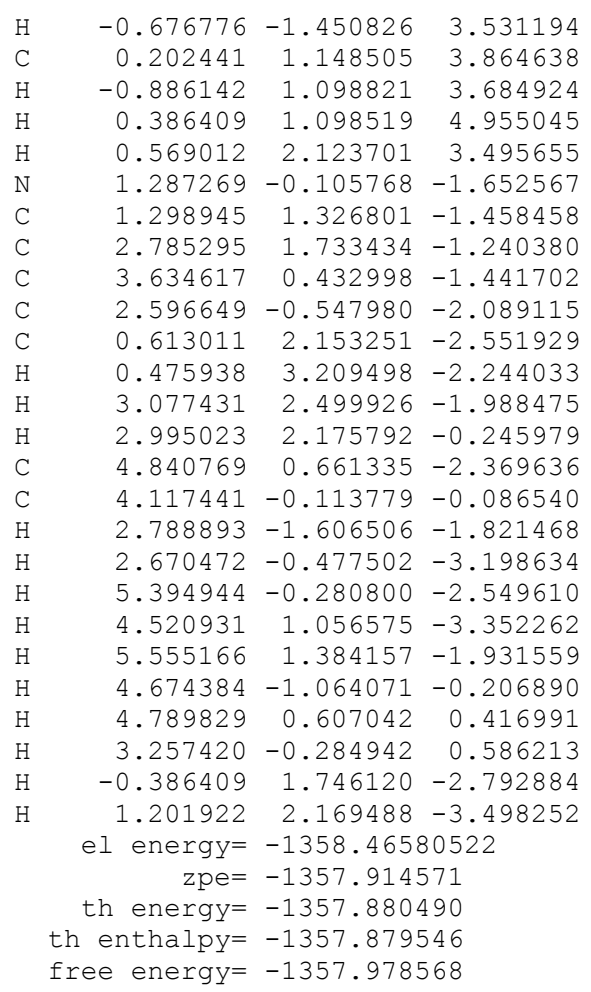

$\begin{array}{lrrr}\text { I3 } & & & \\ \mathrm{Ta} & -0.341465 & -0.063733 & -0.478180 \\ \mathrm{~N} & -2.405818 & -1.551969 & 1.471475 \\ \mathrm{~N} & -2.706706 & -2.547216 & -0.422632 \\ \mathrm{~N} & 1.655439 & 2.734404 & -0.000557 \\ \mathrm{C} & -0.390444 & 1.298373 & 2.461513 \\ \mathrm{~N} & 0.523663 & 2.118681 & 1.731290 \\ \mathrm{C} & -1.954126 & -1.562940 & 0.164084 \\ \mathrm{C} & -0.941294 & 0.268705 & 1.682128 \\ \mathrm{C} & -1.859135 & -0.561827 & 2.341927 \\ \mathrm{C} & -2.665268 & -2.903231 & -1.837342 \\ \mathrm{H} & -3.472211 & -2.383133 & -2.384002 \\ \mathrm{H} & -1.687567 & -2.572756 & -2.228092 \\ \mathrm{C} & 0.777714 & 1.776249 & 0.421232 \\ \mathrm{C} & -2.218951 & -0.416250 & 3.690541 \\ \mathrm{H} & -2.935967 & -1.086513 & 4.179041 \\ \mathrm{C} & -3.588774 & -3.122355 & 0.485382 \\ \mathrm{H} & -4.272927 & -3.919914 & 0.200587 \\ \mathrm{C} & 1.218941 & 3.258590 & 2.111137 \\ \mathrm{H} & 1.148393 & 3.695714 & 3.104668 \\ \mathrm{H} & 3.313429 & 2.732225 & -1.306766 \\ \mathrm{C} & 1.938938 & 3.649337 & 1.011706 \\ \mathrm{H} & 2.614101 & 4.490914 & 0.864821 \\ \mathrm{H} & -2.787607 & -3.994415 & -1.943763 \\ \mathrm{C} & -3.395981 & -2.494373 & 1.689109 \\ \mathrm{H} & -3.874373 & -2.643881 & 2.654383 \\ \mathrm{C} & 2.211794 & 2.794147 & -1.348652 \\ \mathrm{H} & 1.819041 & 1.931965 & -1.909208 \\ \mathrm{H} & 1.910364 & 3.734207 & -1.844129 \\ \mathrm{C} & -0.710987 & 1.507195 & 3.813511 \\ \mathrm{H} & -0.268284 & 2.323289 & 4.396664 \\ \mathrm{C} & -1.629659 & 0.632798 & 4.418197 \\ \mathrm{H} & -1.892086 & 0.771480 & 5.472973 \\ \mathrm{~N} & -1.374624 & 1.013426 & -1.533229 \\ \mathrm{C} & -1.978813 & 1.882695 & -2.506469 \\ \mathrm{C} & -2.067281 & 3.317820 & -1.930150 \\ \mathrm{H} & -2.659797 & 3.319812 & -0.997967 \\ \mathrm{H} & -1.057538 & 3.698943 & -1.692078 \\ \mathrm{H} & -2.540880 & 4.010588 & -2.652003\end{array}$

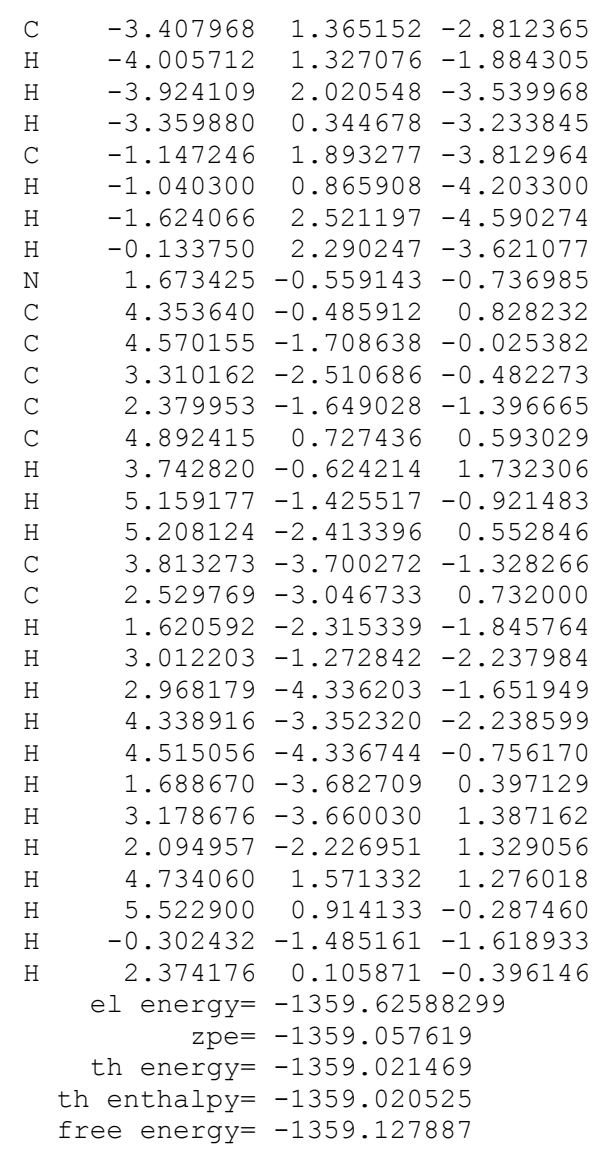

$\begin{array}{lrrr}\text { model-13 } & & & \\ \mathrm{Ta} & -0.164283 & -0.018619 & 0.049188 \\ \mathrm{~N} & 1.939417 & -2.244861 & -0.828144 \\ \mathrm{~N} & 0.160065 & -3.382582 & -0.353654 \\ \mathrm{~N} & 0.003675 & 3.374000 & 0.169813 \\ \mathrm{C} & 2.678349 & 1.299234 & -0.752114 \\ \mathrm{~N} & 1.847222 & 2.407604 & -0.426920 \\ \mathrm{C} & 0.630814 & -2.097221 & -0.414503 \\ \mathrm{C} & 2.000313 & 0.080217 & -0.584386 \\ \mathrm{C} & 2.723573 & -1.064250 & -0.958864 \\ \mathrm{C} & -1.189633 & -3.741349 & 0.058209 \\ \mathrm{H} & -1.169671 & -4.722634 & 0.561311 \\ \mathrm{H} & -1.555068 & -2.976625 & 0.760752 \\ \mathrm{C} & 0.539808 & 2.137635 & -0.076751 \\ \mathrm{C} & 4.040952 & -1.033749 & -1.439835 \\ \mathrm{H} & 4.578675 & -1.947071 & -1.722809 \\ \mathrm{C} & 1.133489 & -4.297384 & -0.741737 \\ \mathrm{H} & 0.945233 & -5.369470 & -0.764818 \\ \mathrm{C} & 2.107481 & 3.769902 & -0.413908 \\ \mathrm{H} & 3.077323 & 4.187744 & -0.674019 \\ \mathrm{H} & -1.357098 & 3.724347 & 1.751379 \\ \mathrm{C} & 0.941234 & 4.381470 & -0.031796 \\ \mathrm{H} & 0.701182 & 5.433883 & 0.110237 \\ \mathrm{H} & -1.868280 & -3.787822 & -0.812083 \\ \mathrm{C} & 2.260412 & -3.576877 & -1.042181 \\ \mathrm{H} & 3.246153 & -3.901482 & -1.367144 \\ \mathrm{C} & -1.352094 & 3.586712 & 0.654512 \\ \mathrm{H} & -1.789191 & 4.475820 & 0.168675 \\ \mathrm{H} & -1.943607 & 2.694611 & 0.397661 \\ \mathrm{C} & 3.994971 & 1.403310 & -1.225021 \\ \mathrm{H} & 4.496676 & 2.371908 & -1.340870 \\ \mathrm{C} & 4.673742 & 0.217140 & -1.558462 \\ \mathrm{H} & 5.704610 & 0.268761 & -1.925632 \\ \mathrm{~N} & -0.102857 & -0.168902 & 1.887544\end{array}$




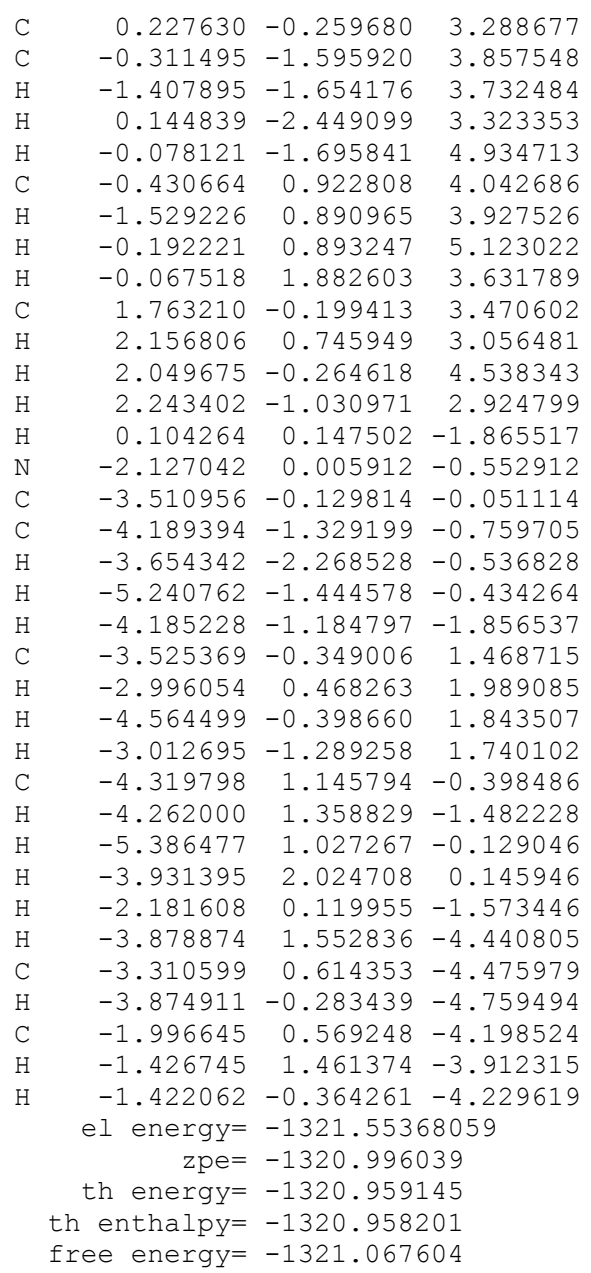

$\begin{array}{lrrr}\text { mTS-13-7 } & & \\ \mathrm{Ta} & 0.235447 & 0.002210 & 0.014202 \\ \mathrm{~N} & -1.953426 & 2.312978 & -0.112027 \\ \mathrm{~N} & -0.071377 & 3.383830 & -0.029531 \\ \mathrm{~N} & 0.008665 & -3.385343 & -0.022344 \\ \mathrm{C} & -2.751460 & -1.221167 & -0.103415 \\ \mathrm{~N} & -1.899856 & -2.359814 & -0.075109 \\ \mathrm{C} & -0.587314 & 2.113414 & -0.064801 \\ \mathrm{C} & -2.010573 & -0.023807 & -0.048248 \\ \mathrm{C} & -2.778647 & 1.155197 & -0.122700 \\ \mathrm{C} & 1.351912 & 3.685043 & 0.039717 \\ \mathrm{H} & 1.499124 & 4.630590 & 0.588016 \\ \mathrm{H} & 1.855225 & 2.866191 & 0.577822 \\ \mathrm{C} & -0.538691 & -2.128022 & -0.050410 \\ \mathrm{C} & -4.176894 & 1.173335 & -0.225173 \\ \mathrm{H} & -4.742187 & 2.111967 & -0.277723 \\ \mathrm{C} & -1.080071 & 4.339641 & -0.074973 \\ \mathrm{H} & -0.867793 & 5.407450 & -0.061504 \\ \mathrm{C} & -2.185860 & -3.716943 & -0.072698 \\ \mathrm{H} & -3.200958 & -4.106198 & -0.101949 \\ \mathrm{H} & 1.708720 & -3.799954 & 1.166222 \\ \mathrm{C} & -0.977719 & -4.365010 & -0.035032 \\ \mathrm{H} & -0.739759 & -5.427383 & -0.019538 \\ \mathrm{H} & 1.785193 & 3.774613 & -0.972195 \\ \mathrm{C} & -2.271653 & 3.662821 & -0.126405 \\ \mathrm{H} & -3.295738 & 4.027738 & -0.154909 \\ \mathrm{C} & 1.434853 & -3.648793 & 0.106092 \\ \mathrm{H} & 1.702122 & -4.546026 & -0.477467 \\ \mathrm{H} & 1.980524 & -2.777263 & -0.285227 \\ \mathrm{C} & -4.148825 & -1.272990 & -0.204855 \\ & & & \end{array}$

$$
\begin{aligned}
& \begin{array}{llll}
-4.692587 & -2.225003 & -0.242007
\end{array} \\
& -4.857355-0.058085-0.256555 \\
& \begin{array}{lll}
-5.950105 & -0.071280 & -0.330376
\end{array} \\
& \begin{array}{lll}
0.859307 & 0.024900 & 1.746854
\end{array} \\
& \begin{array}{lll}
1.114093 & 0.044268 & 3.165993
\end{array} \\
& \begin{array}{lll}
1.973871 & 1.283259 & 3.519550
\end{array} \\
& \begin{array}{lll}
2.941761 & 1.247338 & 2.987292
\end{array} \\
& \begin{array}{lll}
1.447402 & 2.208375 & 3.221575
\end{array} \\
& \begin{array}{lll}
2.176087 & 1.334310 & 4.606391
\end{array} \\
& \begin{array}{lll}
1.878370 & -1.241692 & 3.567380
\end{array} \\
& 2.833304-1.309387 \quad 3.015615 \\
& \begin{array}{lll}
2.098608 & -1.255351 & 4.651850
\end{array} \\
& \begin{array}{lll}
1.272863 & -2.134324 & 3.325638
\end{array} \\
& \begin{array}{rrr}
1.2729635 & 0.110506 & 3.931884
\end{array} \\
& \begin{array}{lll}
-0.857972 & -0.757899 & 3.664908
\end{array} \\
& \begin{array}{lll}
-0.076158 & 0.115091 & 5.028585
\end{array} \\
& \begin{array}{lll}
-0.782406 & 1.024055 & 3.648353
\end{array} \\
& -0.668994-0.003472-1.782075 \\
& \begin{array}{llll}
1.752867 & -0.005562 & -1.329251
\end{array} \\
& \begin{array}{llll}
3.229262 & 0.007902 & -1.407557
\end{array} \\
& \begin{array}{llll}
3.687042 & 1.311899 & -2.107554
\end{array} \\
& 3.415092 \quad 2.193918-1.501787 \\
& \begin{array}{llll}
4.783120 & 1.322746 & -2.259486 \\
3.202171 & 1.410992 & -3.096472
\end{array} \\
& \begin{array}{llll}
3.202171 & 1.410992 & -3.096472
\end{array} \\
& 3.861191-0.072299-0.009536 \\
& \begin{array}{llll}
3.554680 & -0.995934 & 0.513269
\end{array} \\
& 4.964625-0.057636-0.082229 \\
& \begin{array}{lll}
3.536449 & 0.774849 & 0.619818
\end{array} \\
& 3.704652-1.192279-2.263951 \\
& 3.237611-1.162078-3.265769 \\
& 4.803070-1.179999-2.395054 \\
& 3.427722-2.151806-1.791620 \\
& 1.391441-0.011090-2.310872 \\
& 0.857187-0.944828-4.477467 \\
& 0.373929-0.009126-4.172001 \\
& \begin{array}{llll}
0.863502 & 0.923289 & -4.477929
\end{array} \\
& -0.803953-0.005075-3.453298 \\
& -1.395899-0.925396-3.362765 \\
& \begin{array}{lll}
-1.391042 & 0.918537 & -3.364522
\end{array} \\
& \text { el energy }=-1321.54479095 \\
& \mathrm{zpe}=-1320.987736 \\
& \text { th energy }=-1320.951673 \\
& \text { th enthalpy }=-1320.950729 \\
& \text { free energy }=-1321.055720
\end{aligned}
$$

$\begin{array}{lrrr}\text { model-7 } \\ \text { Ta } & -0.453864 & -0.107062 & 0.083117 \\ \mathrm{~N} & 2.402834 & -0.913931 & 1.262125 \\ \mathrm{~N} & 1.399245 & -2.811444 & 0.994509 \\ \mathrm{~N} & -1.869123 & 2.899298 & -0.656568 \\ \mathrm{C} & 1.361254 & 2.515533 & 0.675500 \\ \mathrm{~N} & 0.130305 & 3.033034 & 0.158344 \\ \mathrm{C} & 1.212137 & -1.468158 & 0.834167 \\ \mathrm{C} & 1.351134 & 1.117606 & 0.744027 \\ \mathrm{C} & 2.514939 & 0.513237 & 1.232918 \\ \mathrm{C} & 0.397061 & -3.814448 & 0.653034 \\ \mathrm{H} & 0.373605 & -4.595684 & 1.431764 \\ \mathrm{H} & -0.580390 & -3.309836 & 0.600013 \\ \mathrm{C} & -0.846265 & 2.121548 & -0.193321 \\ \mathrm{C} & 3.648613 & 1.234257 & 1.642221 \\ \mathrm{H} & 4.551309 & 0.742891 & 2.024825 \\ \mathrm{C} & 2.665911 & -3.091763 & 1.497175 \\ \mathrm{H} & 3.002446 & -4.108799 & 1.691374 \\ \mathrm{C} & -0.279407 & 4.335836 & -0.078743 \\ \mathrm{H} & 0.347154 & 5.200693 & 0.127505 \\ \mathrm{H} & -3.947939 & 2.584537 & -0.427369 \\ \mathrm{C} & -1.547975 & 4.251655 & -0.594273 \\ \mathrm{H} & -2.237645 & 5.028771 & -0.919634 \\ \mathrm{H} & 0.624419 & -4.272588 & -0.325983 \\ \mathrm{C} & 3.301680 & -1.887833 & 1.667943 \\ \mathrm{H} & 4.295861 & -1.657014 & 2.043741\end{array}$




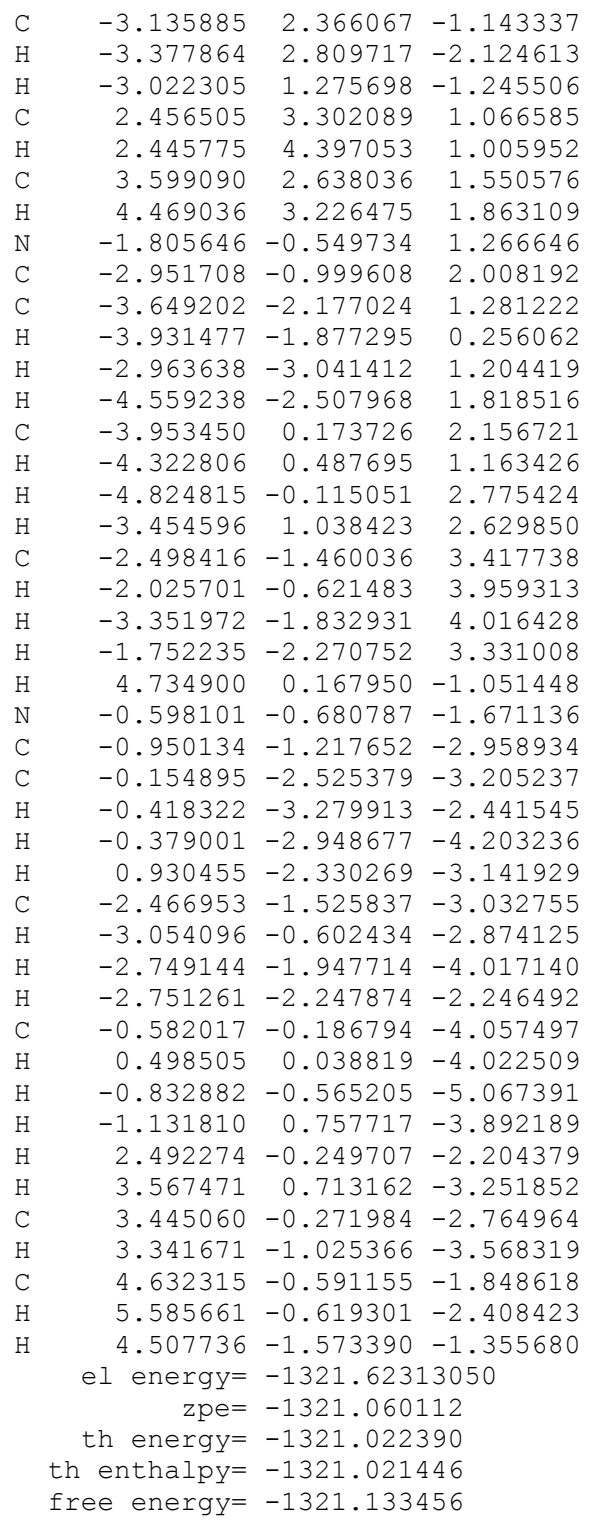

free energy $=-1321.133456$

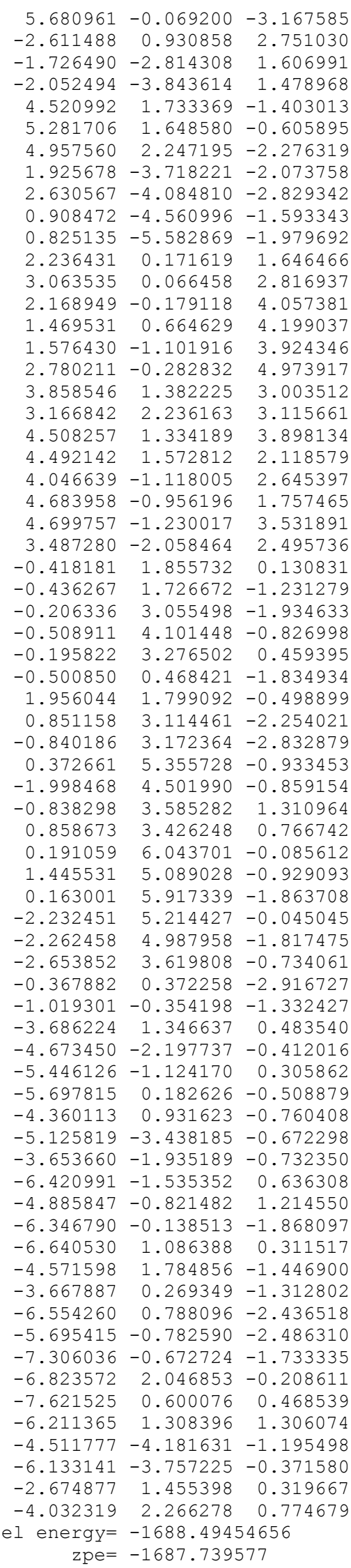

$5.680961-0.069200-3.167585$

$\begin{array}{lll}5.611488 & 0.930858 & 2.751030\end{array}$

1.925678

$2.630567-4.084810-2.829342$

$0.908472-4.560996-1.593343$

$\begin{array}{lll}2.236431 & 0.171619 & 1.646466\end{array}$

$\begin{array}{lll}3.063535 & 0.066458 & 2.816937\end{array}$

$\begin{array}{llll}2.168949 & -0.179118 & 4.057381\end{array}$

$\begin{array}{lll}1.469531 & 0.664629 & 4.199037\end{array}$

$\begin{array}{lll}3.858546 & 1.382225 & 3.003512\end{array}$

$\begin{array}{lll}3.166842 & 2.236163 & 3.115661\end{array}$

$\begin{array}{lll}4.508257 & 1.334189 & 3.898134\end{array}$

$\begin{array}{lll}.508257 & 1.334189 & 3.898134 \\ .092142-1.572812-2.118579\end{array}$

$4.683958-0.956196 \quad 1.757465$

$\begin{array}{lll}4.699757 & -1.230017 & 3.531891\end{array}$

$\begin{array}{llll}3.487280 & -2.058464 & 2.495736\end{array}$

$\begin{array}{lll}-0.418181 & 1.855732 & 0.130831\end{array}$

$\begin{array}{llll}-0.508911 & 4.101448 & -0.826998\end{array}$

$\begin{array}{lll}-0.195822 & 3.276502 & 0.459395\end{array}$

$\begin{array}{lll}1.956044 & 1.799092 & -0.498899\end{array}$

$\begin{array}{llll}0.851158 & 3.114461 & -2.254021\end{array}$

$\begin{array}{llll}-0.840186 & 3.172364 & -2.832879\end{array}$

$\begin{array}{lll}0.372661 & 5.355728 & -0.933453\end{array}$

$\begin{array}{lll}0.858673 & 3.426248 & 0.766742\end{array}$

$\begin{array}{llll}0.191059 & 6.043701 & -0.085612\end{array}$

$\begin{array}{llll}1.445531 & 5.089028 & -0.929093\end{array}$

$0.163001 \quad 5.917339-1.863708$

$-2.232451 \quad 5.214427-0.045045$

$.987958-1.817475$

$\begin{array}{lll}-0.367882 & 0.372258 & -2.916727\end{array}$

$\begin{array}{llll}-1.019301 & -0.354198 & -1.332427\end{array}$

$\begin{array}{llll}-3.686224 & 1.346637 & 0.483540\end{array}$

$\begin{array}{lll}-4.673450 & -2.197737 & -0.412016\end{array}$

$\begin{array}{llll}-5.446126 & -1.124170 & 0.305862\end{array}$

$\begin{array}{lll}-5.697815 & 0.182626 & -0.508879\end{array}$

$\begin{array}{lll}-4.360113 & 0.931623 & -0.760408\end{array}$

$-5.125819-3.438185-0.672298$

$\begin{array}{lll}-6.420991-1.535352 & 0.636308\end{array}$

$\begin{array}{lll}-4.885847 & -0.821482 & 1.214550\end{array}$

$-6.346790-0.138513-1.868097$

$\begin{array}{lll}-6.640530 & 1.086388 & 0.311517\end{array}$

$\begin{array}{lll}-4.571598 & 1.784856 & -1.446900\end{array}$

$\begin{array}{lll}-3.667887 & 0.269349-1.312802\end{array}$

$\begin{array}{llll}-6.554260 & 0.788096 & -2.436518\end{array}$

$\begin{array}{lll}-5.695415 & -0.782590 & -2.486310\end{array}$

$\begin{array}{lll}-6.823572 & 2.046853-0.208611\end{array}$

$\begin{array}{lll}-7.621525 & 0.600076 & 0.468539\end{array}$

$\begin{array}{lll}-6.211365 & 1.308396 & 1.306074\end{array}$

$-4.511777-4.181631-1.195498$

$-6.133141-3.757225-0.371580$

$\begin{array}{lll}-6.133181 & -3.757225 & -0.371580 \\ -2.674877 & 1.455398 & 0.319667\end{array}$

$\begin{array}{rrr}-4.032319 & 2.266278 & 0.774 \\ \text { el energy }= & -1688.49454656\end{array}$ $\mathrm{zpe}=-1687.739577$ 
th energy $=-1687.692904$

th enthalpy $=-1687.691959$

free energy $=-1687.822737$

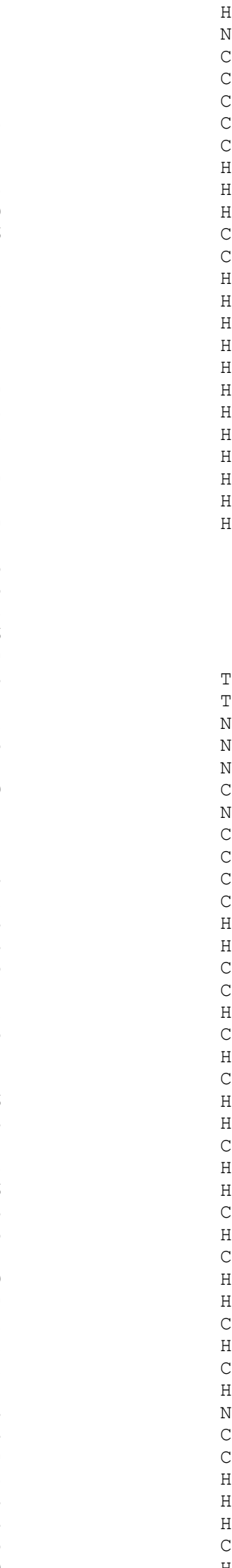

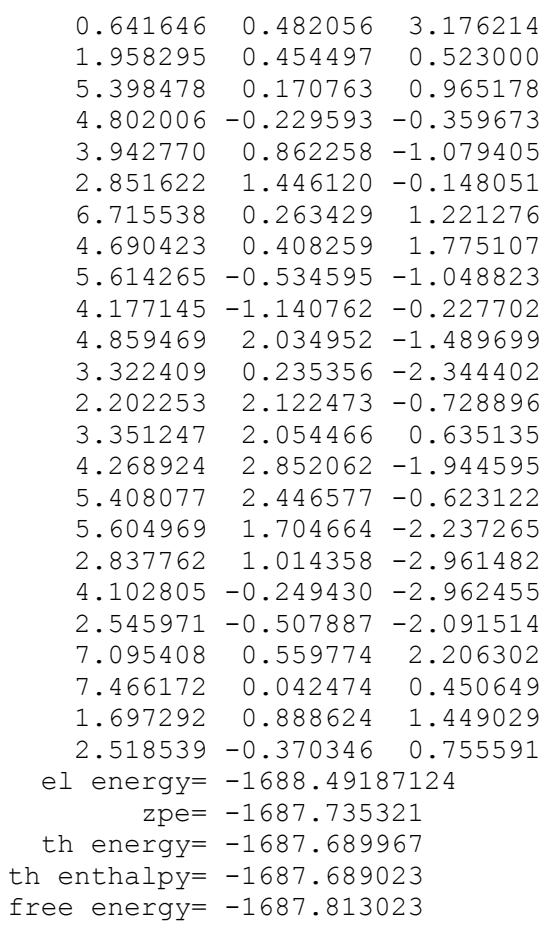




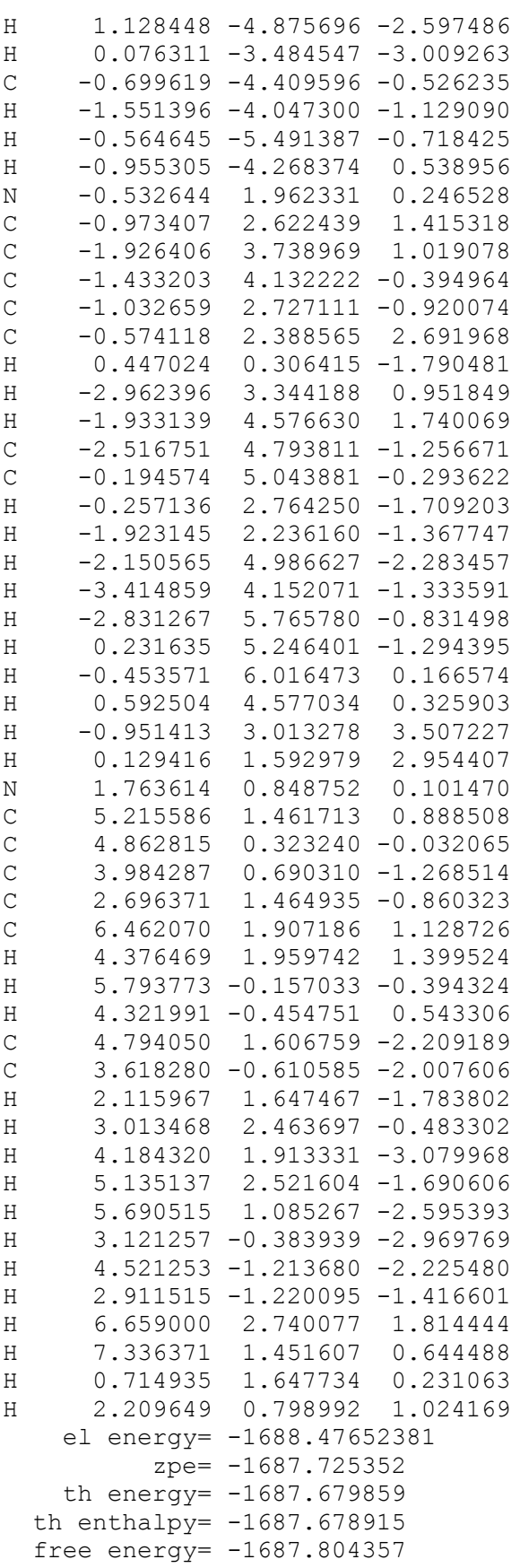

$\begin{array}{lrrr}\text { 13-exchange } & & \\ \mathrm{Ta} & -0.173354 & -0.238358 & -0.234538 \\ \mathrm{~N} & -1.373588 & -1.691883 & 2.396654 \\ \mathrm{~N} & 0.726808 & -2.043608 & 2.719244 \\ \mathrm{~N} & -1.678310 & 0.697175 & -3.163718 \\ \mathrm{C} & -3.406676 & -0.362774 & -0.308419 \\ \mathrm{~N} & -3.051778 & 0.152171 & -1.588066 \\ \mathrm{C} & -0.135671 & -1.404349 & 1.857807 \\ \mathrm{C} & -2.274739 & -0.727910 & 0.438162 \\ \mathrm{C} & -2.547646 & -1.290958 & 1.691841 \\ \mathrm{C} & 2.179516 & -2.054871 & 2.612711 \\ \mathrm{H} & 2.580794 & -2.823702 & 3.292201 \\ \mathrm{H} & 2.475353 & -2.314624 & 1.582517 \\ \mathrm{C} & -1.702918 & 0.283860 & -1.854260 \\ \mathrm{C} & -3.845063 & -1.476531 & 2.199948\end{array}$

$$
\begin{aligned}
& \begin{array}{llll}
-4.024711 & -1.916018 & 3.188121
\end{array} \\
& 0.049435-2.679303 \quad 3.756644 \\
& 0.570551-3.229601 \quad 4.538080 \\
& \begin{array}{llll}
-3.836617 & 0.479481 & -2.680932
\end{array} \\
& \begin{array}{llll}
-4.923452 & 0.441306 & -2.665560
\end{array} \\
& \begin{array}{llll}
0.349298 & 1.116030 & -3.251654
\end{array} \\
& \begin{array}{llll}
-2.963781 & 0.820551 & -3.681285
\end{array} \\
& \begin{array}{llll}
-3.144371 & 1.134354 & -4.708035
\end{array} \\
& 2.601081-1.074092 \quad 2.899288 \\
& \begin{array}{llll}
-1.283667 & -2.457786 & 3.549916
\end{array} \\
& \begin{array}{llll}
-2.154337 & -2.797837 & 4.104750
\end{array} \\
& \begin{array}{llll}
-0.474326 & 0.915271 & -3.956803
\end{array} \\
& -0.235953 \quad 0.013463-4.548692 \\
& \begin{array}{llll}
-0.631635 & 1.772907 & -4.632801
\end{array} \\
& \begin{array}{lll}
-4.728775 & -0.516065 & 0.135615
\end{array} \\
& -5.586893-0.212428-0.475957 \\
& \begin{array}{lll}
-4.933959 & -1.077801 & 1.407359
\end{array} \\
& -5.954321-1.208295 \quad 1.784444 \\
& 0.296925-1.790944-1.104137 \\
& 0.524528-3.071164-1.717937 \\
& 1.933671-3.601977-1.351503 \\
& 2.715653-2.893800-1.680331 \\
& 2.018992-3.734043-0.256793 \\
& 2.131549-4.581349-1.827773 \\
& 0.422631-2.918983-3.257865 \\
& 1.184194-2.207239-3.624654 \\
& 0.573904-3.889026-3.768921 \\
& -0.575390-2.531585-3.531615 \\
& -0.545787-4.078830-1.228972 \\
& -1.556075-3.695982-1.457104 \\
& -0.421696-5.068339-1.709946 \\
& -0.474583-4.210056-0.134123 \\
& \begin{array}{lll}
-0.654204 & 1.674692 & 0.949057
\end{array} \\
& \begin{array}{rrr}
-0.135342 & 2.195408 & 2.101318
\end{array} \\
& \begin{array}{lll}
-0.521833 & 3.669211 & 2.246916
\end{array} \\
& \begin{array}{llll}
-1.071147 & 4.070600 & 0.860233
\end{array} \\
& \begin{array}{lll}
-1.526804 & 2.680424 & 0.329085
\end{array} \\
& \begin{array}{lll}
0.664290 & 1.568429 & 3.046734
\end{array} \\
& \begin{array}{llll}
0.496821 & 0.988418 & -1.390979
\end{array} \\
& \begin{array}{llll}
-1.312700 & 3.755990 & 3.020020
\end{array} \\
& 0.326080 \quad 4.294282 \quad 2.585931 \\
& \begin{array}{llll}
-2.227883 & 5.078231 & 0.933771
\end{array} \\
& 0.061534 \quad 4.627853-0.024021 \\
& \begin{array}{llll}
-1.455043 & 2.625917 & -0.774892
\end{array} \\
& \begin{array}{lll}
-2.595203 & 2.498484 & 0.594593
\end{array} \\
& \begin{array}{llll}
-2.654958 & 5.271994 & -0.069303
\end{array} \\
& \begin{array}{lll}
-3.043938 & 4.704518 & 1.580300
\end{array} \\
& \begin{array}{lll}
-1.891940 & 6.050703 & 1.341918
\end{array} \\
& -0.304760 \quad 4.861046-1.042163 \\
& \begin{array}{llll}
0.482616 & 5.557475 & 0.405123
\end{array} \\
& 0.881345 \quad 3.893415-0.120155 \\
& \begin{array}{lll}
1.025040 & 2.150572 & 3.901309
\end{array} \\
& \begin{array}{lll}
0.641646 & 0.482056 & 3.176214
\end{array} \\
& \begin{array}{lll}
1.958295 & 0.454497 & 0.523000
\end{array} \\
& \begin{array}{llll}
5.398478 & 0.170763 & 0.965178
\end{array} \\
& 4.802006-0.229593-0.359673 \\
& 3.942770 \quad 0.862258-1.079405 \\
& 2.851622 \quad 1.446120-0.148051 \\
& \begin{array}{lll}
6.715538 & 0.263429 & 1.221276
\end{array} \\
& \begin{array}{lll}
4.690423 & 0.408259 & 1.775107
\end{array} \\
& 5.614265-0.534595-1.048823 \\
& 4.177145-1.140762-0.227702 \\
& \begin{array}{lll}
4.859469 & 2.034952 & -1.489699
\end{array} \\
& 3.322409 \quad 0.235356-2.344402 \\
& 2.202253 \quad 2.122473-0.728896 \\
& \begin{array}{lll}
3.351247 & 2.054466 & 0.635135
\end{array} \\
& \begin{array}{lll}
4.268924 & 2.852062-1.944595
\end{array} \\
& \begin{array}{llll}
5.408077 & 2.446577 & -0.623122
\end{array} \\
& \begin{array}{llll}
5.604969 & 1.704664 & -2.237265
\end{array} \\
& 2.837762 \quad 1.014358-2.961482 \\
& 4.102805-0.249430-2.962455
\end{aligned}
$$




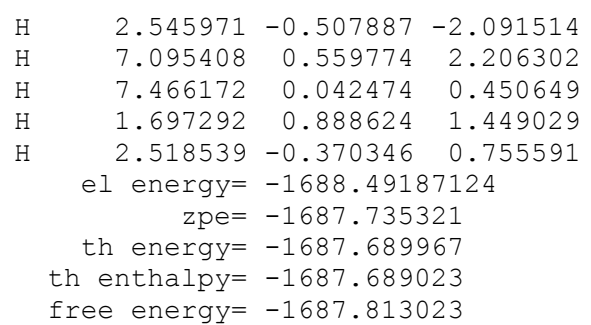

\begin{tabular}{|c|c|c|c|}
\hline \multicolumn{4}{|c|}{ 13-exchange' } \\
\hline & -0.842280 & -0.535686 & 0.400518 \\
\hline N & -1.142018 & -1.373724 & -2.690200 \\
\hline N & -3.055270 & -0.464372 & -2.275430 \\
\hline $\mathbb{N}$ & 1.605184 & -1.170371 & 2.711864 \\
\hline $\mathrm{C}$ & 1.833874 & -1.990938 & -0.688264 \\
\hline $\mathrm{N}$ & 2.104175 & -1.754172 & 0.692211 \\
\hline 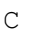 & -1.845884 & -0.729293 & -1.695476 \\
\hline 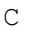 & 0.542048 & -1.580737 & -1.048688 \\
\hline C & 0.184442 & -1.809854 & -2.387011 \\
\hline $\mathrm{C}$ & -4.167949 & 0.176532 & -1.582134 \\
\hline $\mathrm{H}$ & -4.945296 & -0.567445 & -1.330869 \\
\hline $\mathrm{H}$ & -3.775965 & 0.623752 & -0.655567 \\
\hline 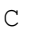 & 1.088215 & -1.188597 & 1.443133 \\
\hline C & 1.039091 & -2.407930 & -3.328507 \\
\hline $\mathrm{H}$ & 0.731891 & -2.578338 & -4.367004 \\
\hline C & -3.111012 & -0.939901 & -3.584331 \\
\hline $\mathrm{H}$ & -3.995906 & -0.825912 & -4.208477 \\
\hline$C$ & 3.215731 & -2.060722 & 1.459547 \\
\hline $\mathrm{H}$ & 4.144974 & -2.434314 & 1.032668 \\
\hline $\mathrm{H}$ & 0.080113 & -0.062659 & 3.576416 \\
\hline C & 2.894708 & -1.692658 & 2.741747 \\
\hline $\mathrm{H}$ & 3.468171 & -1.759675 & 3.664793 \\
\hline $\mathrm{H}$ & -4.606005 & 0.964173 & -2.219252 \\
\hline C & -1.895529 & -1.515712 & -3.847700 \\
\hline $\mathrm{H}$ & -1.521129 & -2.002135 & -4.745621 \\
\hline C & 0.881658 & -0.745842 & 3.905966 \\
\hline $\mathrm{H}$ & 0.432551 & -1.620531 & 4.409760 \\
\hline $\mathrm{H}$ & 1.578021 & -0.238433 & 4.594773 \\
\hline C & 2.742088 & -2.591078 & -1.576618 \\
\hline $\mathrm{H}$ & 3.748690 & -2.888479 & -1.260940 \\
\hline C & 2.323604 & -2.792212 & -2.905465 \\
\hline $\mathrm{H}$ & 3.009487 & -3.259267 & -3.621238 \\
\hline N & -1.999361 & -1.774161 & 1.091296 \\
\hline C & -3.064403 & -2.580202 & 1.624269 \\
\hline C & -3.630867 & -3.487235 & 0.503515 \\
\hline $\mathrm{H}$ & -4.027404 & -2.872223 & -0.325047 \\
\hline $\mathrm{H}$ & -2.833244 & -4.132947 & 0.094968 \\
\hline $\mathrm{H}$ & -4.448066 & -4.130419 & 0.882149 \\
\hline C & -4.191277 & -1.683119 & 2.192947 \\
\hline $\mathrm{H}$ & -4.634801 & -1.065705 & 1.390527 \\
\hline $\mathrm{H}$ & -4.998425 & -2.288373 & 2.648738 \\
\hline $\mathrm{H}$ & -3.783792 & -1.001103 & 2.959900 \\
\hline C & -2.491788 & -3.466789 & 2.759735 \\
\hline $\mathrm{H}$ & -2.092833 & -2.832630 & 3.571987 \\
\hline $\mathrm{H}$ & -3.271622 & -4.128432 & 3.183227 \\
\hline $\mathrm{H}$ & -1.667520 & -4.092825 & 2.374348 \\
\hline $\mathrm{N}$ & 4.207113 & 0.486475 & -1.452740 \\
\hline C & 5.195522 & 0.094934 & -0.552262 \\
\hline C & 5.163738 & 1.088809 & 0.600259 \\
\hline C & 4.250326 & 2.250817 & 0.114380 \\
\hline C & 3.324785 & 1.506930 & -0.885930 \\
\hline C & 6.023256 & -0.977717 & -0.669613 \\
\hline $\mathrm{H}$ & -0.659057 & 0.422270 & 1.938418 \\
\hline $\mathrm{H}$ & 4.712720 & 0.596160 & 1.485424 \\
\hline $\mathrm{H}$ & 6.172923 & 1.429848 & 0.893986 \\
\hline C & 3.453710 & 2.897731 & 1.256308 \\
\hline C & 5.087316 & 3.311976 & -0.628268 \\
\hline $\mathrm{H}$ & 2.941196 & 2.176931 & -1.679485 \\
\hline H & 2.444868 & 1.070461 & -0.362473 \\
\hline
\end{tabular}

\begin{tabular}{|c|c|c|c|}
\hline & 2.750349 & 3.660453 & 0.872119 \\
\hline & 2.859627 & 2.145453 & 1.807924 \\
\hline $\mathrm{H}$ & 4.127191 & 3.399350 & 1.976592 \\
\hline $\mathrm{H}$ & 4.442392 & 4.118368 & -1.024988 \\
\hline $\mathrm{H}$ & 5.827427 & 3.775691 & 0.050463 \\
\hline $\mathrm{H}$ & 5.634326 & 2.862525 & -1.476408 \\
\hline & 6.812989 & -1.150245 & 0.067017 \\
\hline $\mathrm{H}$ & 5.989885 & -1.643952 & -1.539133 \\
\hline $\mathrm{N}$ & -1.118492 & 1.475805 & -0.123881 \\
\hline & -2.135370 & 4.658876 & -1.459584 \\
\hline & -2.879135 & 4.010412 & -0.321705 \\
\hline & -2.023718 & 3.648925 & 0.933286 \\
\hline C & -0.816803 & 2.731138 & 0.557726 \\
\hline C & -2.353590 & 5.904139 & -1.919310 \\
\hline $\mathrm{H}$ & -1.355630 & 4.050752 & -1.944421 \\
\hline $\mathrm{H}$ & -3.710214 & 4.671451 & -0.003705 \\
\hline & -3.351299 & 3.073357 & -0.685868 \\
\hline & -1.460986 & 4.938107 & 1.562983 \\
\hline $\mathrm{C}$ & -2.931985 & 2.931730 & 1.948990 \\
\hline $\mathrm{H}$ & -0.281817 & 2.481217 & 1.492153 \\
\hline $\mathrm{H}$ & -0.113588 & 3.340823 & -0.056997 \\
\hline & -0.835146 & 4.703540 & 2.444717 \\
\hline $\mathrm{H}$ & -0.841237 & 5.503202 & 0.842703 \\
\hline $\mathrm{H}$ & -2.275844 & 5.607271 & 1.899935 \\
\hline $\mathrm{H}$ & -2.383410 & 2.726278 & 2.886889 \\
\hline $\mathrm{H}$ & -3.819146 & 3.545604 & 2.198632 \\
\hline $\mathrm{H}$ & -3.274853 & 1.957648 & 1.555934 \\
\hline $\mathrm{H}$ & -1.782483 & 6.323012 & -2.756706 \\
\hline $\mathrm{H}$ & -3.118355 & 6.553835 & -1.472393 \\
\hline $\mathrm{H}$ & 3.796367 & -0.226280 & -2.055713 \\
\hline & -1.487843 & 1.682526 & -1.058081 \\
\hline & el energy= & -1688.4871 & 11429 \\
\hline & $\mathrm{zpe}=$ & -1687.732 & 765 \\
\hline & th energy= & -1687.6859 & 914 \\
\hline & enthalpy= & -1687.6849 & 970 \\
\hline & ee energy= & -1687.8172 & 207 \\
\hline \multicolumn{4}{|c|}{ 13-exchange' ' } \\
\hline $\mathrm{Ta}$ & -1.166799 & -0.256781 & -0.212757 \\
\hline $\mathrm{N}$ & -0.560866 & 0.893079 & 2.734471 \\
\hline $\mathrm{N}$ & -0.215059 & -1.219144 & 3.005402 \\
\hline $\mathrm{N}$ & -2.458451 & 1.219802 & -3.017822 \\
\hline $\mathrm{C}$ & -1.648469 & 2.939583 & -0.070617 \\
\hline $\mathrm{N}$ & -2.033919 & 2.586905 & -1.398955 \\
\hline $\mathrm{C}$ & -0.556471 & -0.302808 & 2.049164 \\
\hline $\mathrm{C}$ & -1.274068 & 1.816863 & 0.682038 \\
\hline $\mathrm{C}$ & -0.915803 & 2.074079 & 2.013840 \\
\hline $\mathrm{C}$ & -0.107870 & -2.654516 & 2.769577 \\
\hline $\mathrm{H}$ & -0.848380 & -3.197558 & 3.382676 \\
\hline $\mathrm{H}$ & -0.312361 & -2.836586 & 1.704231 \\
\hline C & -1.955862 & 1.250147 & -1.742527 \\
\hline $\mathrm{C}$ & -0.919127 & 3.357762 & 2.584618 \\
\hline $\mathrm{H}$ & -0.634012 & 3.530186 & 3.628783 \\
\hline $\mathrm{C}$ & -0.016258 & -0.616380 & 4.245966 \\
\hline $\mathrm{H}$ & 0.264387 & -1.184700 & 5.131120 \\
\hline $\mathrm{C}$ & -2.558207 & 3.358241 & -2.421464 \\
\hline $\mathrm{H}$ & -2.693274 & 4.434828 & -2.346240 \\
\hline $\mathrm{H}$ & -1.977897 & -0.758008 & -3.415746 \\
\hline $\mathrm{C}$ & -2.831338 & 2.488884 & -3.446419 \\
\hline $\mathrm{H}$ & -3.255620 & 2.663219 & -4.433758 \\
\hline $\mathrm{H}$ & 0.908021 & -3.008380 & 3.018983 \\
\hline $\mathrm{C}$ & -0.234656 & 0.724870 & 4.072197 \\
\hline $\mathrm{H}$ & -0.179914 & 1.549198 & 4.779312 \\
\hline $\mathrm{C}$ & -2.662386 & 0.011828 & -3.810676 \\
\hline $\mathrm{H}$ & -3.704364 & -0.340610 & -3.707595 \\
\hline $\mathrm{H}$ & -2.447108 & 0.229097 & -4.870432 \\
\hline $\mathrm{C}$ & -1.667963 & 4.248926 & 0.432252 \\
\hline $\mathrm{H}$ & -1.961118 & 5.106548 & -0.184505 \\
\hline $\mathrm{C}$ & -1.296521 & 4.441349 & 1.774652 \\
\hline $\mathrm{H}$ & -1.300856 & 5.452913 & 2.195648 \\
\hline $\mathrm{N}$ & -2.741063 & -1.120587 & 0.113465 \\
\hline
\end{tabular}




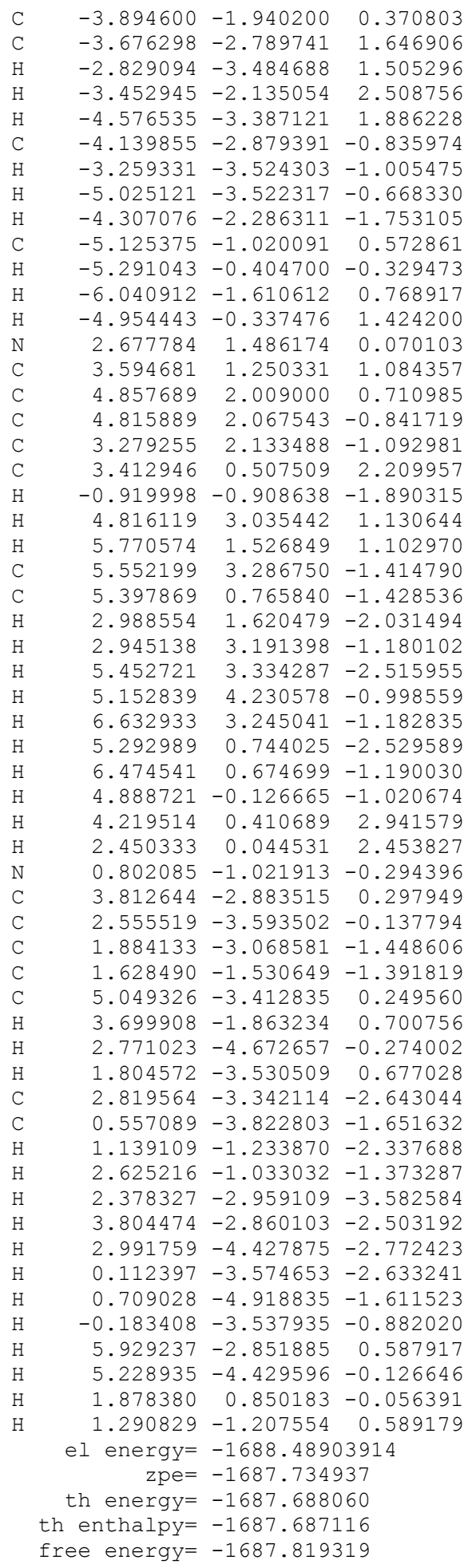

$\begin{array}{lrrr}14 & & & \\ \mathrm{Ta} & -0.023647 & 0.253861 & 0.098995 \\ \mathrm{~N} & -2.884931 & 0.198557 & -1.231904 \\ \mathrm{~N} & -2.357797 & 2.308497 & -1.180390 \\ \mathrm{~N} & 2.061649 & -2.130980 & 1.225662 \\ \mathrm{C} & -0.984890 & -2.778954 & -0.397163\end{array}$

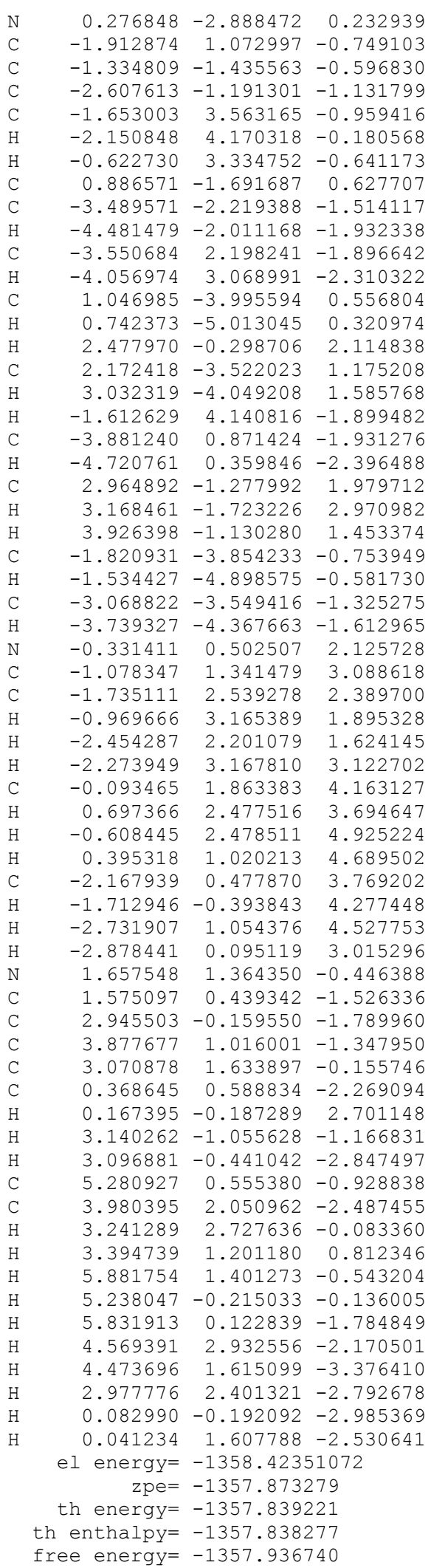

